\title{
Measurement of Metabolic Monitoring in Youth and Adult Medicaid Recipients Prescribed Antipsychotics
}

\author{
Gail A. Edelsohn, MD, MSPH; Meghna Parthasarathy, MS; Lauren Terhorst, PhD; \\ Irina O. Karpov, MS; and James Schuster, MD, MBA
}

\begin{abstract}
BACKGROUND: Individuals being treated with first- and second-generation antipsychotics (FGAs and SGAs) are at risk for a variety of adverse cardiometabolic effects. Although consensus guidelines that recommend metabolic monitoring for patients receiving SGAs have been in place since 2004, the rate of monitoring remains low, especially in the pediatric population.
\end{abstract}

OBJECTIVES: To (a) examine differences in rates of laboratory monitoring for glucose and lipids for adults and youth prescribed FGAs and SGAs; (b) look at factors associated with the likelihood of metabolic testing; and (c) describe cohort effects that may have had an impact on the rates of laboratory testing.

METHODS: This is a retrospective study examining the rates of glucose and lipid testing for 3 separate cohorts of Medicaid recipients who were prescribed antipsychotics during 3 measurement periods-2008, 2010, and 2012-using paid Medicaid pharmacy and laboratory claims data. The sample included adults aged 18 years and older and children aged 17 years and younger. For each measurement period, we identified the rate of metabolic monitoring and the demographic characteristics for each individual, including race, age, and gender. The proportion of laboratory monitoring was assessed using chi square tests for each of the outcomes. Logistic regression models for each time point were used to determine the characteristics of individuals who were more likely to receive monitoring.

RESULTS: The proportion of individuals receiving glucose and lipid tests increased for both age groups across all measurement periods. For individuals aged 18 years and over, glucose monitoring increased from $56.6 \%$ $72.6 \%$. Testing for lipids remained constant, ranging from $38.3 \%-41.2 \%$ for each of the 3 measurement periods. During the first measurement period, in 2008 , females were $41 \%$ and $15 \%$ more likely to receive glucose and lipid laboratory monitoring, respectively, compared with males. Females continued to be more likely to receive glucose monitoring during the measurement periods in 2010 and 2012, although there was no significant difference between females and males for lipid monitoring during these time periods. Individuals aged 17 years and younger were $59 \%-68 \%$ less likely to receive glucose monitoring than adults (aged $\geq 18$ years) for all time points. Across all measurement periods, individuals aged $\leq 17$ years were also $44 \%-58 \%$ less likely to receive lipid monitoring compared with adults (aged $\geq 18$ years). While there was no significant difference between Caucasians and non-Caucasians in the first measurement period, Caucasians were about $30 \%$ less likely to receive glucose monitoring and about $50 \%$ less likely to receive lipid monitoring during the measurement periods covering 2010 and 2012.

CONCLUSIONS: Metabolic monitoring in adults improved substantially over the time periods studied; however, rates remained suboptimal, especially in the pediatric population. This finding suggests that interventions to increase metabolic monitoring in adults and children using FGAs and SGAs are necessary.

J Manag Care Spec Pharm. 2015;21(9):769-77

Copyright $\odot 2015$, Academy of Managed Care Pharmacy. All rights reserved.

\section{What is already known about this subject}

Comorbidity of medical conditions is high for individuals with mental health diagnoses, and the potential cardiometabolic adverse effects from antipsychotic medications may be exacerbated in the presence of other risk factors.

Guidelines for metabolic monitoring focus on individuals with prescriptions for second-generation antipsychotics (SGAs). Less attention is given to the metabolic effects of first-generation antipsychotics (FGAs)

Despite the existence of guideline consensus recommendations for metabolic monitoring of SGAs, screening rates for glucose and lipids remain suboptimal for adults and children whose treatment services are funded by Medicaid.

\section{What this study adds}

The proportion of adults receiving glucose testing significantly increased with each successive measurement period, with $56 \%$ of adults receiving tests in 2008 and $79 \%$ in 2012. The change in the proportion of adults receiving lipid testing for each measurement period was not significant.

The proportion of youth receiving glucose testing increased significantly with each successive measurement period, with $30 \%$ of youth receiving tests in 2008 and $50 \%$ in 2012. The rate for youth receiving lipid lipid testing showed a significant increase, from $19 \%$ in 2008 to $28 \%$ in 2012

During the study period, there were cohort effects, including a change in the prior authorization policies for antipsychotics for those enrolled in Medicaid fee-for-service plans, as well as educational interventions developed by the public behavioral health managed care organization that targeted prescribers and members, that may have had a positive impact on rates of testing for glucose and lipids.

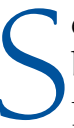
econd-generation antipsychotics (SGAs) have increasingly been prescribed for children and adults for a variety of psychiatric disorders. ${ }^{1-3}$ When the time period 1993-1998 was compared with that of 2005-2009, office-based antipsychotic visits for children increased from 0.024 per 100 to 1.83 per 100; for adolescents from 0.78 per 100 to 3.76 per 100; and for adults from 3.25 per 100 to 6.18 per $100 .{ }^{1}$ National trends data examining office-based visits for youth found that 
children were 6 times more likely to receive prescriptions for antipsychotics in 2010 than in 1995 (odds ratio $=6.0$, 95\% confidence interval $=3.90-9.26){ }^{2}$ While SGAs have clinical efficacy for a number of conditions, they also carry the risk of adverse effects, including cardiometabolic, endocrine, and neurologic side effects. ${ }^{4-8}$ Although the use of first-generation antipsychotics (FGAs) is not as prevalent as SGAs, FGAs are still used in treatment and have been associated with weight gain, hyperglycemia, and insulin resistance. ${ }^{9}$

The U.S. Food and Drug Administration mandated a class warning for all SGAs in 2003 that required all product labeling to reference the risk of hyperglycemia and diabetes mellitus, as well as the potential for extreme hyperglycemia associated with ketoacidosis, hyperosmolar coma, or death. ${ }^{5}$ In early 2004, the American Diabetes Association (ADA), the American Psychiatric Association (APA), the North American Association of the Study of Obesity, and the American Association of Clinical Endocrinologists issued a consensus recommendation for metabolic monitoring for individuals who have been prescribed SGAs. ${ }^{6}$ At the time that the consensus recommendations were issued, there were limited data regarding the risks of diabetes in children and adolescents taking SGAs.

Given the shortened life expectancy of individuals with serious mental illness, chiefly due to cardiovascular disease, ${ }^{10}$ the potential for adverse effects from psychotropic medication may be exacerbated when combined with other risk factors such as obesity, unhealthy lifestyle, and exposure to adverse childhood experiences. An association between schizophrenia and metabolic abnormalities has been noted prior to treatment with antipsychotics, and multiple studies have found that the rates of metabolic monitoring at baseline and follow-up for those prescribed SGAs remain low and fall short of recommended guidelines. ${ }^{10-13}$

Haupt et al. (2009) examined a large managed care database that measured lipid and glucose testing before and after the release of the ADA/APA guidelines and found improvements after implementation of the guidelines; however, rates remained low and those for the pediatric population were lowest. ${ }^{14} \mathrm{~A}$ study of commercially insured patients found similar results, with only $13 \%$ of patients being given glucose and lipid tests within 6 months of beginning treatment with SGAs. ${ }^{15}$ Raebel et al. (2014) utilized a national electronic health care database for youth newly starting SGAs and found that only $11 \%$ had been given glucose assessment between 90 days before and 30 days after first dispensing, with some variation depending on the medication used, the site, the age group, and gender. ${ }^{16}$

FGAs are included in the data reported here because these medications are also implicated in cardiometabolic side effects. The METEOR study, a cross-sectional study that looked at the prevalence of metabolic disorders and hypertension in patients with schizophrenia, compared the prevalence of these disorders in patients being treated with FGAs and SGAs. ${ }^{17}$ Patients were stratified by the type of antipsychotic they were receiving (FGAs or SGAs), and data collection was done at 1 point in time. The investigators found no difference in the prevalence of glycemic disorders, dyslipidemia, or metabolic syndrome for patients with schizophrenia between the group taking FGAs and the group taking SGAs. The prevalence of hypertension was higher in those treated with FGAs. The proportion of females (but not males) who were overweight or obese was higher in the group treated with SGAs. ${ }^{17}$

A historic cohort study that compared the incidence of metabolic syndrome in first-episode patients with schizophrenia treated with either FGAs or SGAs found the rates of metabolic syndrome over the course of 3 years increased for both groups; however, the incidence was 3 times higher for those taking SGAs compared with those taking FGAs. ${ }^{18}$ While, as a class, SGAs are recognized as increasing the risk of metabolic abnormalities, there is considerable variation in the adverse effects on metabolic indices among the various SGAs. In this cohort study, when patients being treated with clozapine and olanzapine-SGAs that are associated with a significantly negative impact on metabolic indicators-were excluded from the results, the difference between the 2 groups was no longer significant. ${ }^{18}$

Community Care Behavioral Health Organization, a nonprofit recovery-oriented behavioral health managed care organization and part of the University of Pittsburgh Medical Center Insurance Division, identified metabolic monitoring as a quality improvement priority and began measuring the rates of metabolic monitoring in its membership in 2007. In this study, we describe trends in metabolic monitoring for adult and pediatric Medicaid recipients who have been prescribed FGAs or SGAs. We compared the rates of laboratory monitoring for glucose and lipids at 3 measurement times for adults (aged $\geq 18$ years) and youth (aged $\leq 17$ years). We also examined the change in monitoring rates across 3 time periods, controlling for confounding variables. We described the demographic characteristics of individuals who were least likely to receive laboratory monitoring. The difference in monitoring rates between those prescribed FSAs and SGAs was also examined across all measurement periods.

\section{Methods}

\section{Design}

This is a retrospective study that examined the rates of glucose and lipid testing for 3 separate cohorts of Medicaid recipients prescribed antipsychotics during 2008, 2010, and 2012 using paid Medicaid pharmacy and laboratory claims data. This study was conducted as part of a quality improvement initiative, consistent with the National Committee for Quality Assurance guidelines. The Institutional Review Board determined that this study did not need to undergo review. 


\section{FIGURE 1 Sample Selection}

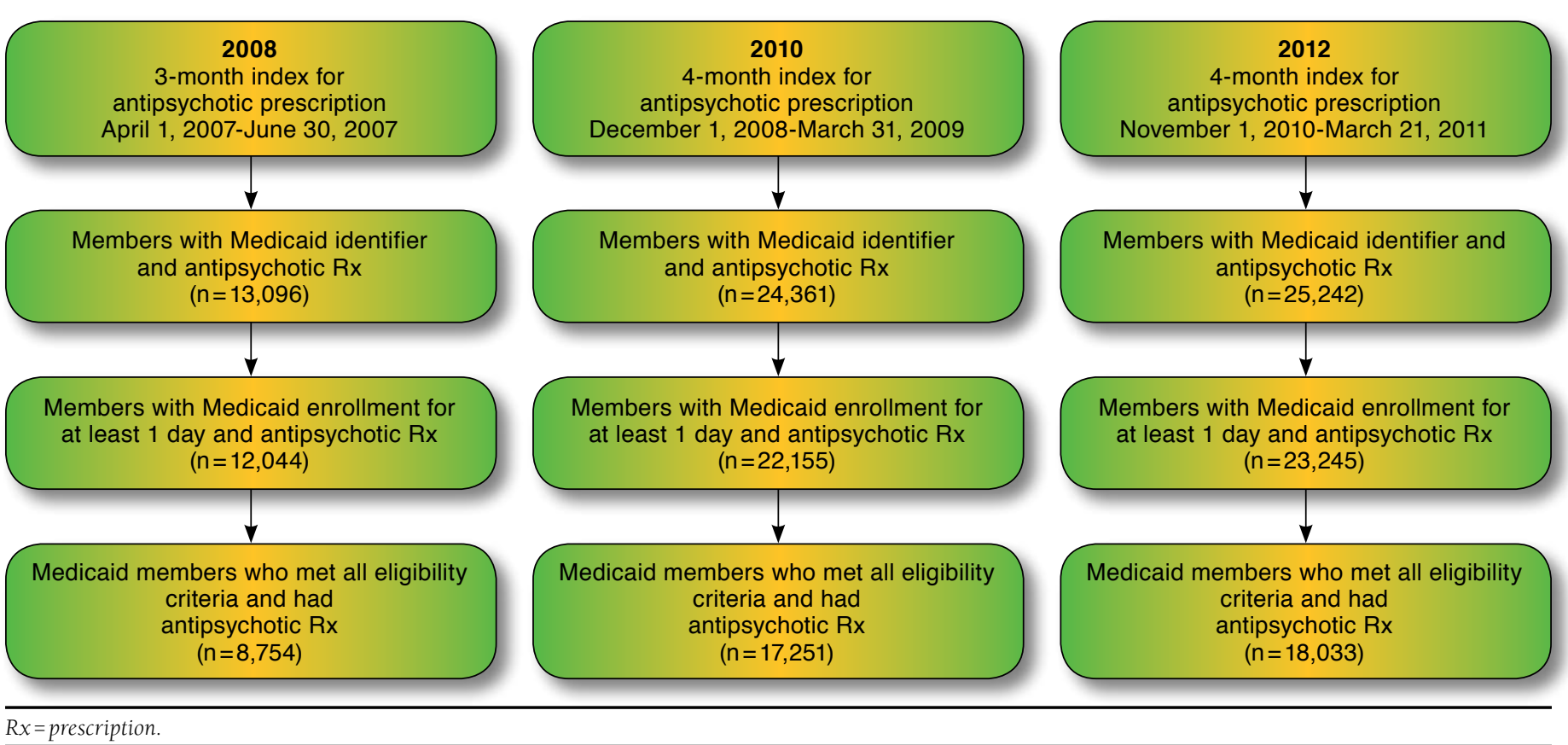

\section{Study Population}

The population included adults (defined as aged 18 years and older) and youth (defined as aged 17 years and younger) who were Medicaid recipients. Members with Medicare or commercial primary insurance were excluded from the analysis. The study population reflected 3 separate measurement periods: measurement period 1 (2008), measurement period 2 (2010), and measurement period 3 (2012). For each measurement period, the denominator was the number of Medicaid recipients enrolled in a public behavioral health managed care organization who filled an antipsychotic prescription during a 3- or 4-month time period. The time frame used to identify Medicaid recipients was increased from 3 months for the analysis in 2008 to 4 months for the analyses in 2010 and 2012. This change was made to ensure that individuals filling a 90-day supply of medication were included in the study.

The numerator was the count of all members who met denominator criteria and who received a glucose or lipids test during the 18- or 19-month time period that was used to capture laboratory information. The time period measuring laboratory monitoring was extended from 12 months to 18 or 19 months to give a generous time frame that would allow for the capture of all laboratory claims. The final sample for each measurement period had to have met Medicaid eligibility for 12 of the 18- or 19-month period. For 2008, 12,044 Medicaid members had an antipsychotic prescribed dur- ing the 3-month period, with 8,754 meeting all eligibility criteria. In 2010, 22,155 Medicaid members had an antipsychotic prescribed during a 4-month period, with 17,251 members meeting all eligibility criteria. In 2012, 23,245 Medicaid members had an antipsychotic prescribed during a 4-month period, with 18,033 members meeting all eligibility criteria (Figure 1).

\section{Measurements}

The top 5 diagnoses were obtained utilizing the International Classification of Diseases, Ninth Revision, Clinical Modification (ICD-9-CM) by grouping codes into broad diagnostic categories. The crosswalk between the ICD-9-CM codes and the broader diagnosis categories is shown in the Appendix (available in online article). The top 5 behavioral health diagnoses represent the most commonly assigned diagnoses for adults and children for each of the 3 cohorts in 2008, 2010, and 2012. These diagnoses are included here to provide some clinical context for the study population. These diagnostic categories were assigned during all the service visits that occurred during the measurement time periods. The diagnosis category that was most commonly assigned was considered the final diagnosis for the individual. Once each individual was assigned a single diagnosis in this manner, a count of unique individuals by diagnosis was generated for the diagnosis distribution (Tables 1 and 2). 
TABLE 1 Distribution of Primary Diagnoses for Children (Aged $\leq 17$ Years) During Specified Measurement Period (Top 5 Diagnoses Only)

\begin{tabular}{|c|c|c|c|c|c|c|}
\hline \multirow[b]{2}{*}{ Diagnosis } & \multicolumn{2}{|c|}{2008} & \multicolumn{2}{|c|}{2010} & \multicolumn{2}{|c|}{2012} \\
\hline & $\mathbf{n}$ & $\%$ & $\mathbf{n}$ & $\%$ & n & $\%$ \\
\hline ADHD & 818 & 32.15 & 1,541 & 34.26 & 1,350 & 31.45 \\
\hline Autism & 223 & 8.77 & 466 & 10.36 & 534 & 12.44 \\
\hline Bipolar & 178 & 7.00 & 275 & 6.11 & 292 & 6.80 \\
\hline Dysthymia/depression NOS & 212 & 8.33 & 432 & 9.60 & 450 & 10.48 \\
\hline ODD & 201 & 7.90 & 443 & 9.85 & 425 & 9.90 \\
\hline
\end{tabular}

$A D H D=$ attention-deficit/hyperactivity disorder; $N O S=$ not otherwise specified; ODD = oppositional defiance disorder.

TABLE 2 Distribution of Primary Diagnoses for Adults (Aged $\geq 18$ Years) During Specified Measurement Period (Top 5 Diagnoses Only)

\begin{tabular}{|c|c|c|c|c|c|c|}
\hline \multirow[b]{2}{*}{ Diagnosis } & \multicolumn{2}{|c|}{2008} & \multicolumn{2}{|c|}{2010} & \multicolumn{2}{|c|}{2012} \\
\hline & n & $\%$ & $\mathbf{n}$ & $\%$ & $\mathbf{n}$ & $\%$ \\
\hline Bipolar & 813 & 13.09 & 2,130 & 16.70 & 2,468 & 17.96 \\
\hline Dysthymia/depression NOS & 430 & 6.92 & 1,147 & 8.99 & 1,263 & 9.19 \\
\hline Major depression & 780 & 12.56 & 1,744 & 13.68 & 1,999 & 14.55 \\
\hline Schizophrenia & 846 & 13.62 & 1,258 & 9.86 & 1,230 & 8.95 \\
\hline Schizoaffective & 662 & 10.66 & 1,020 & 8.00 & 1,043 & 7.59 \\
\hline
\end{tabular}

Descriptive statistics summarized the study population's characteristics (gender, age group, and race). Two dependent variables-rates of lipids test and rates of glucose test-were created to assess rates of laboratory claims during the 3 measurement periods. A dichotomous variable for lipid tests was coded "l" if an individual received a lipids test within the specified measurement times and " 0 " if the individual did not receive a lipids test within the specified measurement period. The glucose test outcome was a dichotomous variable coded " 1 " if the individual received a glucose test within the specified measurement period and "0" if the individual did not receive a glucose test within the specified measurement period. Independent variables were gender, age group, race, and antipsychotic type. Antipsychotic type was coded "1" for FGA and "0" for SGA. The FGA group included individuals prescribed only FGAs during the study period. The SGA group included those prescribed either only SGAs or FGAs and SGAs during the study period.

Differences in proportions of laboratory monitoring over the 3 time periods were assessed using separate chi square tests of association for each of the outcomes. Logistic regression models were created for each measurement period and for each outcome in order to determine characteristics of individuals who were more likely to receive laboratory tests. All statistical analyses were performed using SAS, version 9.4 (SAS Institute, Inc., Carey, NC), with a significance level of 0.05 .

\section{Results}

The final study samples for the 3 measurement periods were as follows: (a) measurement period 1 (2008) consisted of 8,754 Medicaid recipients (6,210 adults, 2,544 youth); (b) measurement period 2 (2010) consisted of 17,251 Medicaid recipients (12,753 adults, 4,498 youth); and (c) measurement period 3 (2012) consisted of 18,033 Medicaid recipients (13,740 adults, 4,293 youth). The sample was mostly Caucasian, ranging from nearly $61 \%$ in 2008 to about $74 \%$ in 2010 and 2012. Gender distribution was even throughout the 3 measurement periods (Table 3).

The proportion of individuals receiving glucose and lipid tests was significantly different between the age groups during each measurement period (Table 4). The proportion of youth receiving glucose testing was $30 \%$ in $2008,41 \%$ in 2010 , and $50 \%$ in 2012 . The proportion of youth receiving lipid testing was $20 \%$ in $2008,22 \%$ in 2010 , and $28 \%$ in 2012 . The proportion of adults receiving lipid testing was 38\% in 2008, $40 \%$ in 2010 , and $41 \%$ in 2012 . The proportion of adults receiving glucose testing was $57 \%$ in 2008, 70\% in 2010, and 73\% in 2012.

The results of the logistic regression models for each measurement period are displayed in Table 5. During measurement period 1 , the odds of females receiving glucose and lipid tests were $41 \%$ and $15 \%$ higher, respectively, compared with males. Females continued to be more likely to receive glucose monitoring through measurement periods 2 and 3 (Table 5); however, there was no significant difference between females and 


\section{TABLE 3 Demographics}

\begin{tabular}{|c|c|c|c|c|c|c|}
\hline & \multicolumn{2}{|c|}{$\begin{array}{c}\text { Measurement } 1 \\
(2008) \\
n=8,754\end{array}$} & \multicolumn{2}{|c|}{$\begin{array}{c}\text { Measurement } 2 \\
(2010) \\
\mathrm{n}=17,251\end{array}$} & \multicolumn{2}{|c|}{$\begin{array}{c}\text { Measurement } 3 \\
(2012) \\
\mathbf{n}=18,033\end{array}$} \\
\hline & $\mathrm{n}$ & $\%$ & $\mathrm{n}$ & $\%$ & $\mathrm{n}$ & $\%$ \\
\hline \multicolumn{7}{|l|}{ Age group } \\
\hline $0-17$ & 2,544 & 29.06 & 4,498 & 26.07 & 4,293 & 23.81 \\
\hline $18-64$ & 6,210 & 70.94 & 12,753 & 73.93 & 13,740 & 76.19 \\
\hline \multicolumn{7}{|l|}{ Race } \\
\hline African American & 2,148 & 25.07 & 2,707 & 15.69 & 2,746 & 15.23 \\
\hline Other & 1,248 & 14.26 & 1,044 & 10.69 & 1,894 & 10.50 \\
\hline Caucasian & 5,311 & 60.67 & 12,700 & 73.62 & 13,393 & 74.27 \\
\hline \multicolumn{7}{|l|}{ Gender } \\
\hline Female & 4,265 & 48.72 & 8,446 & 48.96 & 8,831 & 48.97 \\
\hline Male & 4,489 & 51.28 & 8,805 & 51.04 & 9,202 & 51.03 \\
\hline
\end{tabular}

males for lipid monitoring during measurement periods 2 or 3 . The odds of individuals aged 17 years and younger receiving glucose tests were 59\%-62\% lower compared with adults aged 18 years and over for each measurement period.

Similarly, for all measurement periods, the odds of individuals aged 17 years and youger receiving lipid labs were 44\%-58\% lower compared with adults aged 18 years and over. While there was no significant difference between Caucasians and non-Caucasians during measurement period 1, Caucasians were about $30 \%$ less likely to receive glucose testing and about $50 \%$ less likely to receive lipid testing for measurement periods 2 and 3. During measurement period 2, individuals using FGAs were 29\% more likely to receive glucose monitoring and $22 \%$ more likely to received lipid monitoring.

\section{Discussion}

SGAs are widely used for a variety of psychiatric conditions, and FGAs are also still in use, largely for the treatment of schizophrenia. ${ }^{19}$ Both types of medication carry significant risks for cardiometabolic side effects, and metabolic monitoring is suggested for people utilizing these antipsychotics. However, this monitoring is often neglected, putting these individuals at risk for life-threatening complications. We studied the demographics of those receiving laboratory monitoring over a period of 6 years to determine who was least likely to receive monitoring.

In our study, we found that rates of metabolic laboratory monitoring increased for children and adults over time. During measurement period 2, the rates were higher for glucose testing (70\%) than for lipid testing (40\%), a finding similar to that reported in other studies. ${ }^{11,14,15,20}$ There was a significant increase in the rate of laboratory monitoring over time in our Medicaid population, unlike the results reported in the 3-state study by Morrato et al. (2010). ${ }^{12}$ While these increases were statistically significant, a noteworthy proportion of the population was still not receiving the recommended testing by the end of the study.

Females were more likely to receive testing for glucose during all measurement periods. There is limited literature addressing differences between the genders in adherence to medical recommendations. A study of adult type 2 diabetic patients found ethnic and gender differences in social support, acceptance of diabetes, quality of life, and adherence behaviors. ${ }^{21}$ Raebel et al.'s 2014 study of pediatric patients newly begun on SGAs found that females were more likely to receive glucose testing at baseline. ${ }^{16}$

While there was no difference between Caucasians and non-Caucasians during measurement period 1 in our study, Caucasians were less likely to receive laboratory testing in the 2 subsequent cohorts. Youth were less likely than adults to receive monitoring for glucose and lipids during the 3 measurement periods. Measurement period 2 was the only period where the likelihood of receiving glucose and lipid monitoring was affected by the type of antipsychotic drug being used. During measurement period 2, those patients prescribed FGAs were significantly more likely to receive laboratory testing than those prescribed SGAs only or SGAs with FGAs.

There are several factors that could explain the lower rates of monitoring in youth. Unlike adults, children and adolescents depend on their guardians to implement monitoring recommendations. Guardians may be reluctant to expose children and adolescents to blood draws. The likelihood of metabolic monitoring increases with age, which may make it less likely that individuals aged 17 years and under will be monitored. ${ }^{15,22}$

TABLE 4 Laboratory Test Distributions and Comparisons by Age Group from 2008 to 2012

\begin{tabular}{|c|c|c|c|c|c|c|c|c|c|c|}
\hline \multirow{3}{*}{$\begin{array}{l}\text { Laboratory } \\
\text { Test }\end{array}$} & \multicolumn{5}{|c|}{ Aged $\leq 17$ Years } & \multicolumn{5}{|c|}{ Aged $\geq 18$ Years } \\
\hline & $\begin{array}{c}2008 \\
(n=2,544)\end{array}$ & $\begin{array}{c}2010 \\
(n=4,498)\end{array}$ & $\begin{array}{c}2012 \\
(n=4,293)\end{array}$ & \multirow[b]{2}{*}{$\chi^{2}(\mathrm{df}=2)$} & \multirow[b]{2}{*}{$P$ Value } & $\begin{array}{c}2008 \\
(n=6,210)\end{array}$ & $\begin{array}{c}2010 \\
(\mathrm{n}=12,753)\end{array}$ & $\begin{array}{c}2012 \\
(n=13,740)\end{array}$ & \multirow[b]{2}{*}{$\chi^{2}(\mathrm{df}=2)$} & \multirow[b]{2}{*}{$P$ Value } \\
\hline & \multicolumn{3}{|c|}{ n (\%) } & & & \multicolumn{3}{|c|}{ n (\%) } & & \\
\hline Glucose & $\begin{array}{c}781 \\
(30.70) \\
\end{array}$ & $\begin{array}{c}1,847 \\
(41.06) \\
\end{array}$ & $\begin{array}{c}2,145 \\
(49.97)\end{array}$ & 246.56 & $<0.0001$ & $\begin{array}{c}3,515 \\
(56.60) \\
\end{array}$ & $\begin{array}{c}8,904 \\
(69.82) \\
\end{array}$ & $\begin{array}{c}9,980 \\
(72.63) \\
\end{array}$ & 526.47 & $<0.0001$ \\
\hline Lipid & $\begin{array}{c}499 \\
(19.61)\end{array}$ & $\begin{array}{c}1,011 \\
(22.48)\end{array}$ & $\begin{array}{c}1,222 \\
(28.46)\end{array}$ & 79.17 & $<0.0001$ & $\begin{array}{c}2,380 \\
(38.33)\end{array}$ & $\begin{array}{c}5,076 \\
(39.80)\end{array}$ & $\begin{array}{c}5,666 \\
(41.24)\end{array}$ & 15.99 & 0.0003 \\
\hline
\end{tabular}

$\mathrm{df}=$ degree of freedom; $\chi 2=$ Pearson's chi square test . 


\begin{tabular}{|c|c|c|c|c|c|c|c|c|c|c|c|c|c|}
\hline \multirow{2}{*}{\multicolumn{2}{|c|}{ Laboratory Test }} & \multicolumn{3}{|c|}{$\beta$} & \multicolumn{3}{|c|}{ Standard Error } & \multicolumn{3}{|c|}{$x^{2}$} & \multicolumn{3}{|c|}{ OR $(95 \% \mathrm{CI})$} \\
\hline & & M1 & M2 & M3 & M1 & M2 & M3 & M1 & M2 & M3 & M1 & M2 & M3 \\
\hline \multirow{6}{*}{ Glucose } & Intercept & -0.05 & 0.93 & 1.03 & 0.07 & 0.06 & 0.06 & 0.47 & $276.97^{\mathrm{a}}$ & $328.42^{\mathrm{a}}$ & & & \\
\hline & $\begin{array}{l}\text { Gender } \\
(\text { female }=1, \text { male }=0)\end{array}$ & 0.34 & 0.29 & 0.44 & 0.05 & 0.03 & 0.03 & $57.34^{\mathrm{a}}$ & $75.16^{\mathrm{a}}$ & $168.41^{\mathrm{a}}$ & $\begin{array}{c}1.41 \\
(1.29-1.54) \\
\end{array}$ & $\begin{array}{c}1.34 \\
(1.25-1.43) \\
\end{array}$ & $\begin{array}{c}1.55 \\
(1.45-1.65) \\
\end{array}$ \\
\hline & $\begin{array}{l}\text { Race } \\
(\text { white }=1, \text { other }=0)\end{array}$ & 0.12 & -0.34 & -0.33 & 0.07 & 0.05 & 0.06 & 3.40 & $37.91^{\mathrm{a}}$ & $34.98^{a}$ & $\begin{array}{c}1.13 \\
(0.99-1.29) \\
\end{array}$ & $\begin{array}{c}0.71 \\
(0.64-0.80) \\
\end{array}$ & $\begin{array}{c}0.72 \\
(0.65-0.80) \\
\end{array}$ \\
\hline & $\begin{array}{l}\text { Race } \\
(\text { black =1, other =0) }\end{array}$ & 0.15 & -0.06 & -0.18 & 0.07 & 0.07 & 0.07 & $4.27 \mathrm{~b}$ & 0.71 & $10.19 \mathrm{~b}$ & $\begin{array}{c}1.17 \\
(1.01-1.35) \\
\end{array}$ & $\begin{array}{c}0.95 \\
(0.83-1.08)\end{array}$ & $\begin{array}{c}0.84 \\
(0.73-0.96) \\
\end{array}$ \\
\hline & $\begin{array}{l}\text { Age } \\
(<18=1, \geq 18=0)\end{array}$ & -0.97 & -1.13 & -0.89 & 0.05 & 0.04 & 0.04 & 352.28 & $922.24^{a}$ & $559.67^{a}$ & $\begin{array}{c}0.38 \\
(0.34-0.42) \\
\end{array}$ & $\begin{array}{c}0.32 \\
(0.30-0.35) \\
\end{array}$ & $\begin{array}{c}0.41 \\
(0.38-0.44) \\
\end{array}$ \\
\hline & $\begin{array}{l}\text { Antipsychotic type } \\
(\mathrm{FGA}=1, \mathrm{SGA}=0)\end{array}$ & 0.10 & 0.26 & -0.07 & 0.15 & 0.11 & 0.07 & 0.45 & $5.94^{b}$ & 0.95 & $\begin{array}{c}1.10 \\
(0.83-1.47) \\
\end{array}$ & $\begin{array}{c}1.29 \\
(1.05-1.59) \\
\end{array}$ & $\begin{array}{c}0.93 \\
(0.81-1.08) \\
\end{array}$ \\
\hline \multirow{6}{*}{ Lipids } & Intercept & -0.66 & 0.05 & 0.15 & 0.07 & 0.05 & 0.05 & $82.46^{\mathrm{a}}$ & 0.87 & $9.02^{b}$ & & & \\
\hline & $\begin{array}{l}\text { Gender } \\
(\text { female }=1, \text { male }=0)\end{array}$ & 0.14 & -0.02 & 0.03 & 0.05 & 0.03 & 0.03 & $8.33 \mathrm{~b}$ & 0.35 & 0.62 & $\begin{array}{c}1.15 \\
(1.05-1.26)\end{array}$ & $\begin{array}{c}0.98 \\
(0.92-1.05)\end{array}$ & $\begin{array}{c}1.03 \\
(0.96-1.09)\end{array}$ \\
\hline & $\begin{array}{l}\text { Race } \\
(\text { white }=1 \text {, other }=0 \text { ) }\end{array}$ & 0.10 & -0.64 & -0.70 & 0.07 & 0.05 & 0.05 & 2.16 & $151.71^{\mathrm{a}}$ & $196.98^{a}$ & $\begin{array}{c}1.11 \\
(0.97-1.28) \\
\end{array}$ & $\begin{array}{c}0.53 \\
(0.48-0.58) \\
\end{array}$ & $\begin{array}{c}0.50 \\
(0.45-0.55) \\
\end{array}$ \\
\hline & $\begin{array}{l}\text { Race } \\
(\text { black=1, other=0) }\end{array}$ & 0.15 & 0.06 & -0.02 & 0.08 & 0.06 & 0.06 & 3.40 & 0.92 & 0.10 & $\begin{array}{c}1.16 \\
(0.99-1.35) \\
\end{array}$ & $\begin{array}{c}1.06 \\
(0.94-1.20) \\
\end{array}$ & $\begin{array}{c}0.98 \\
(0.87-1.10) \\
\end{array}$ \\
\hline & $\begin{array}{l}\text { Age } \\
(<18=1, \geq 18=0)\end{array}$ & -0.88 & -0.85 & -0.58 & 0.06 & 0.04 & 0.04 & $227.26^{\mathrm{a}}$ & $416.21^{\mathrm{a}}$ & $211.42^{\mathrm{a}}$ & $\begin{array}{c}0.42 \\
(0.37-0.47) \\
\end{array}$ & $\begin{array}{c}0.43 \\
(0.39-0.46) \\
\end{array}$ & $\begin{array}{c}0.56 \\
(0.52-0.61) \\
\end{array}$ \\
\hline & $\begin{array}{l}\text { Antipsychotic type } \\
(\mathrm{FGA}=1, \mathrm{SGA}=0)\end{array}$ & 0.19 & 0.20 & -0.02 & 0.15 & 0.09 & 0.07 & 1.74 & $4.37^{b}$ & 0.12 & $\begin{array}{c}1.21 \\
(0.91-1.62) \\
\end{array}$ & $\begin{array}{c}1.22 \\
(1.01-1.47) \\
\end{array}$ & $\begin{array}{c}0.98 \\
(0.85-1.12) \\
\end{array}$ \\
\hline \multicolumn{14}{|c|}{$\begin{array}{l}a P<0.0001 \text {. } \\
b 0.0001 \leq P<0.05 \text {. } \\
\beta=\text { beta, the regression coefficient } ; C I=\text { confidence interval; } F G A=\text { first-generation antipsychotic; } M 1=\text { measurement period } 1 ; M 2=\text { measurement period } 2 ; M 3=\text { measure- } \\
\text { ment period 3; } O R=\text { odds ratio; } S G A=\text { second-generation antipsychotic; } \chi 2=\text { Pearson's chi square test. }\end{array}$} \\
\hline
\end{tabular}

Morrato et al. (2010) have shown that children with serious or multiple psychiatric diagnoses who use health care services more intensively were more likely to receive metabolic screening. ${ }^{13}$ A study of the determinants of metabolic testing in a Medicaid population prescribed antipsychotics found that, for children and adults, comorbid diabetes and dyslipidemia were predictors for glucose and lipid testing. ${ }^{23}$

While the association between SGA use and type 2 diabetes in children is now known, the prevalence of these disorders in youth compared with adults has not yet been determined. Since our claims data were restricted to psychiatric diagnoses, we could not examine the impact of diabetes or lipid disorders on testing for youth or adults in our study. In the literature, there has been far less attention paid to laboratory monitoring in children using antipsychotics than monitoring in adults using antipsychotics. There are currently no pediatric guidelines for metabolic monitoring when prescribing antipsychotics in the United States. Pringsheim et al. (2011) have published guidelines specific to pediatric treatment in Canada. ${ }^{24}$

Adults who are non-Caucasian, older, and/or who have preexisting diagnoses of diabetes, dyslipidemia, and hypertension have a greater likelihood of undergoing general glucose and lipid monitoring. ${ }^{14,25-27}$ In 1 study of metabolic monitoring rates for Medicaid recipients diagnosed with schizophrenia, younger individuals (aged 18-30 years) and males were less likely to be tested for glucose levels. ${ }^{28}$ The same study found a higher likelihood of testing for lipids was associated with urban residence as well as comorbid diabetes and dyslipidemia. ${ }^{28}$ Since African Americans are considered more at risk for metabolic abnormalities, there is a possibility that during the study period, these individuals were already being closely monitored. The population of African Americans in our study is clustered in 1 county that has access to an academic institution and a high level of care, which may explain their higher levels of testing.

Cohort effects may have had an impact on rates of metabolic monitoring during our study period. Notably, there were changes in the Pennsylvania Department of Public Welfare prior authorizations for fee-for-service programs regarding antipsychotic medication in youth. In September 2009, clinical prior authorization was required for all antipsychotic medication prescribed to children aged under 6 years, and this policy was progressively expanded to cover all youth under the age 18 years by November 2012. By January 2012, clinical prior authorization required documentation of ongoing laboratory, blood pressure, and involuntary movement disorder monitoring in children. Some managed care organizations developed prior authorization policies that were consistent with the state Medicaid fee-for-service prior authorization policies. A study 
comparing the effects of prior authorization polices on children from Pennsylvania with those from another mid-Atlantic state enrolled in Medicaid found that prior authorization polices had a modest but statistically significant effect on antipsychotic use, decreasing use in children aged 6-12 years but having no impact on younger children. ${ }^{29}$ Our study did not examine the impact of prior authorizations on laboratory monitoring for metabolic syndrome.

During our study period, Community Care Behavioral Health Organization implemented several educational interventions with providers. These interventions included letters to prescribers that gave details on metabolic monitoring rates for members in their geographic regions, recommendations for metabolic monitoring, and a free webinar focused on health management when prescribing antipsychotics. Provider alerts that incorporated the ADA/APA consensus guidelines and delineated expectations for providers regarding follow-up and interventions were issued in October 2011 and January 2012. ${ }^{6}$ Members enrolled in the schizophrenia disease management program received educational outreach regarding the risk of developing metabolic syndrome provided by care managers. Members received written material recommending a discussion with their behavioral health providers and primary care doctors about blood tests and weight monitoring while on such medication.

Barriers to management of physical health conditions and consistent laboratory monitoring in individuals with serious mental illness include physician-related factors such as poor communication with the patient or difficulty maintaining communication between mental health providers and primary care providers. Physicians also do not always accept established guidelines. Service-related factors also play a role; a lack of clearly designated responsibility for recognizing and managing physical health problems and lack of integrated services can affect monitoring rates. ${ }^{30} \mathrm{~A}$ study of Australian mental health services found that $69 \%$ of mental health center staff members were unsure about which care providers should follow up on abnormal cardiometabolic screening results. ${ }^{31}$ A lack of communication between behavioral health care providers and physical health care providers regarding the member's treatment can result in negative health consequences associated with inadequate laboratory monitoring.

Guidelines often do not provide clarity on which provider should assume responsibility for metabolic monitoring and interventions based on the results of the monitoring. A survey of psychiatrists found that the majority were aware of the need for metabolic monitoring. ${ }^{30}$ Psychiatrists who completed their residencies prior to the 2004 ADA/APA guidelines, and those who had a minority of patients with diagnoses of schizophrenia or bipolar disorder were more likely to agree that monitoring is the role of psychiatrists only if patients did not have primary care providers. ${ }^{32,33}$ Haupt et al. found that a higher number of primary care visits increased the rate of monitoring, indicat- ing the benefit of assigning a clinician to follow up on general physical health in individuals being treated with SGAs. ${ }^{14}$

From a managed care perspective, we have observed that there is limited information sharing across treatment settings, which may make it difficult to track metabolic monitoring across all levels of care. Primary care providers may not have access to the laboratory studies obtained in psychiatric inpatient settings; residential, drug, and alcohol treatment settings; or in outpatient services. While electronic health records can provide such information, access to these records may be limited to a particular level of care or a specific provider or health system. Efforts to further integrate behavioral health and physical health care could include mechanisms for sharing data that would facilitate the coordination and accountability of tracking metabolic monitoring for those treated with antipsychotic medication. A reluctance to expose youth and adults to repeated testing and concerns about the cost of obtaining testing could be mitigated to some extent if there were full access for all parties to laboratory testing results obtained in different settings.

\section{Limitations}

There are some limitations to this study. First, claims data do not capture pharmacy or laboratory data on individuals receiving inpatient psychiatric services, and this study was limited to individuals in the majority of counties in a Mid-Atlantic state that were enrolled in Medicaid. Second, the monitoring rates presented here may be overestimated in reference to ADA/APA guidelines, which specify that fasting plasma glucose and lipid measures be taken at baseline and thereafter quarterly for the first year, with glucose measurements repeated annually after the first year and lipid measurements repeated every 5 years. ${ }^{6}$ Our tests were obtained within 18-19 months rather than the 12 months recommended by the ADA/APA guidelines. We chose this time frame because it is likely that, in our population, many individuals were continuing on antipsychotics and were not new starts, in which case the 12-month monitoring interval recommended by the ADA/APA would not reflect the time frame of psychopharmacologic interventions for our population. In addition, we were aware of the barriers to obtaining blood work in a timely manner and so examined the rates of obtaining metabolic measurements within a more generous time window. Third, our focus was on laboratory testing and not on other components of metabolic monitoring, such as physical measures (i.e., body mass index, waist circumference, and blood pressure) or personal or family histories. As discussed earlier, we did not have access to physical health diagnoses claims data, so we could not examine the impact of comorbidities on the rate of laboratory testing. Finally, our use of cohorts in 3 time periods limited the possibility of generalizing the finding of an effect over time. 


\section{Conclusions}

The current findings suggest that, for adults and children receiving FGAs and SGAs, interventions are needed to encourage monitoring of glucose and lipid levels. This is particularly important in the pediatric population, where monitoring was lowest in our study and in the literature. As in other studies, our study found that African Americans were more likely to be monitored. However, as mentioned previously, our result may be due to a population of African Americans in a specific county where health care resources were more available than elsewhere. Managed care organizations do have a role to play in promoting metabolic monitoring. Interventions could address (a) ways in which members and their providers could work more closely to ensure that monitoring lab work is obtained; (b) enhancing access to information for patients receiving antipsychotic medications; (c) increasing integration of behavioral and physical health care; and (d) educating providers about the need for metabolic monitoring in any patient receiving antipsychotic medication. Our findings also support the need for future longitudinal investigations of laboratory monitoring.

\section{Authors}

GAIL A. EDELSOHN, MD, MSPH, is Senior Medical Director; MEGHNA PARTHASARATHY, MS, is Systems Analyst; IRINA O. KARPOV, MS, is Clinical Outcomes Analyst; and JAMES SCHUSTER, MD, MBA, is Chief Medical Officer, Community Care Behavioral Health Organization, Pittsburgh, Pennsylvania. LAUREN TERHORST, PhD, is Associate Professor, Department of Occupational Therapy and School of Nursing, Health, and Community Systems, School of Health and Rehabilitation Sciences, Pittsburgh, Pennsylvania.

AUTHOR CORRESPONDENCE: Gail A. Edelsohn, MD, MSPH, Community Care Behavioral Health Organization, 1 E. Uwchlan Ave., Ste. 311, Exton, PA 19341. Tel.: 215.601.6266; Fax: 888.589.6559; E-mail: edelsohnga@ccbh.com.

\section{REFERENCES}

1. Olfson M, Blanco C, Liu SM, Wang S, Correll CU. National trends in the office-based treatment of children, adolescents, and adults with antipsychotics. Arch Gen Psychiatry. 2012;69(12):1247-56. Available at: http://archpsyc.jamanetwork.com/article.aspx?articleid=1263977. Accessed July 30, 2015.

2. Olfson M, Blanco C, Wang S, Laje G, Correll CU. National trends in the mental health care of children, adolescents, and adults by office-based physicians. JAMA Psychiatry. 2014;71(1):81-90. Available at: http://archpsyc jamanetwork.com/article. aspx?articleid=1784344. Accessed July 30, 2015

3. Medicaid Medical Directors Learning Network and Rutgers Center for Education and Research on Mental Health Therapeutics. Antipsychotic medication use in Medicaid children and adolescents: report and resource guide from a 16-state study. Medicaid Medical Directors Learning Network/ Rutgers CERTs Publication No. 1. June 2010. Available at: http://rci.rutgers. edu/ cseap/MMDLNAPKIDS.html. Accessed July 30, 2015.

4. De Hert M, Dekker JM, Wood D, Kahl KG, Holt RI, Möller HJ. Cardiovascular disease and diabetes in people with severe mental illness position statement from the European Psychiatric Association (EPA), supported by the European Association for the Study of Diabetes (EASD) and the European Society of Cardiology (ESC). Eur Psychiatry. 2009;24(6):412-24 Available at: http://www.europsy-journal.com/article/S0924-9338\%2809\% 2900017-0/fulltext. Accessed July 30, 2015.

5. Rosack J. FDA to require diabetes warning on antipsychotics. Psychiatric News. October 17, 2003. Available at: http://psychnews.psychiatryonline.org/ doi/full/10.1176/pn.38.20.0001a. Accessed July 30, 2015.

6. American Diabetes Association; American Psychiatric Association; American Association of Clinical Endocrinologists; North American Association for the Study of Obesity. Consensus development conference on antipsychotic drugs and obesity and diabetes. J Clin Psychiatry. 2004;65(2):267-72

7. Newcomer JW, Hennekens $\mathrm{CH}$. Severe mental illness and risk of cardiovascular disease. JAMA. 2007;298(15):1794-96.

8. Correll CU, Manu P, Olshanskiy V, Napolitano B, Kane JM, Malhotra AK. Cardiometabolic risk of second-generation antipsychotic medications during first-time use in children and adolescents. JAMA. 2009;302(16):1765-73. Available at: http://jama.jamanetwork.com/article.aspx?articleid=184782. Accessed July 30, 2015

9. Chadda RK, Ramshankar P, Deb KS, Sood M. Metabolic syndrome in schizophrenia: differences between antipsychotic-naïve and treated patients. J Pharmacol Pharmacother. 2013 Jul;4(3):176-86. Available at: http://www. jpharmacol. .com/article. asp?issn=0976-500X; year $=2013$; volume $=4$; issue $=3 ;$ sp age $=176$; epage $=186$; aulast $=$ Chadda. Accessed July 30, 2015 .

10. Colton CW, Manderscheid RW. Congruencies in increased mortality rates years of potential life lost, and causes of death among public mental health clients in eight states. Prev Chronic Dis. 2006;3(2):A42. Available at: http:// www.cdc.gov/pcd/issues/2006/apr/05_0180.htm. Accessed July 30, 2015.

11. Mitchell AJ, Delaffon V, Vancampfort D, Correll CU, De Hert M. Guideline concordant monitoring of metabolic risk in people treated with antipsychotic medication: systematic review and meta-analysis of screening practices. Psychol Med. 2012;42(1):125-47.

12. Morrato EH, Druss B, Hartung DM, et al. Metabolic testing rates in 3 state Medicaid programs after FDA warnings and ADA/APA recommendations for second-generation antipsychotic drugs. Arch Gen Psychiatry. 2010;67(1):17-24. Available at: http://archpsyc.jamanetwork.com/article. aspx?articleid=210512. Accessed July 30, 2015.

13. Morrato EH, Nicol GE, Maahs D, et al. Metabolic screening in children receiving antipsychotic drug treatment. Arch Pediatr Adolesc Med. 2010;164(4):344-51. Available at: http://archpedi.jamanetwork.com/article. aspx?articleid=383055. Accessed July 30, 2015. 
14. Haupt DW, Rosenblatt LC, Kim E, Baker RA, Whitehead R, Newcomer JW. Prevalence and predictors of lipid and glucose monitoring in commercially insured patients treated with second-generation antipsychotic agents. Am J Psychiatry. 2009;166(3):345-53. Available at: http://ajp.psychiatryonline.org/ doi/full/10.1176/appi.ajp.2008.08030383. Accessed July 30, 2015.

15. Morrato EH, Newcomer JW, Kamat S, Baser O, Harnett J, Cuffel B. Metabolic screening after the American Diabetes Association's consensus statement on antipsychotic drugs and diabetes. Diabetes Care. 2009;32(6):1037-42. Available at: http://care.diabetesjournals.org/content/32/6/1037.long. Accessed July 30, 2015.

16. Raebel MA, Penfold R, McMahon AW, et al. Adherence to guidelines for glucose assessment in starting second-generation antipsychotics. Pediatrics. 2014;134(5):e1308-14

17. Falissard B, Mauri M, Shaw K, et al. The METEOR study: frequency of metabolic disorders in patients with schizophrenia. Focus on first and second generation and level of risk of antipsychotic drugs. Int Clin Psychopharmacol. 2011;26(6):291-302.

18. De Hert M, Schreeurs V, Sweers K et al. Typical and atypical antipsychotics differentially affect long-term incidence rates of the metabolic syndrome in first-episode patients with schizophrenia: a retrospective chart review. Schizophr Res. 2008;101(1-3):295-303.

19. Lieberman JA, Stroup TS, McEvoy JP, et al.; Clinical Antipsychotic Trials of Intervention Effectiveness (CATIE) Investigators. Effectiveness of antipsychotic drugs in patients with chronic schizophrenia. N Engl J Med. 2005;353(12):1209-23. Available at: http://www.nejm.org/doi/full/10.1056/ NEJMoa051688. Accessed July 30, 2015

20. Dhamane AD, Martin BC, Brixner DI, Hudson TJ, Said Q. Metabolic monitoring of patients prescribed second-generation antipsychotics. J Psychiatr Pract. 2013;19(5):360-74.

21. Misra R, Lager J. Ethnic and gender differences in psychosocial factors, glycemic control, and quality of life among adult type 2 diabetic patients. J Diabetes Complications. 2009;23(1):54-64.

22. Thompson A, Hetrick SE, Alvarez-Jimenez M, et al. Targeted intervention to improve monitoring of antipsychotic-induced weight gain and metabolic disturbance in first episode psychosis. Aust NZ Psychiatry. 2011;45(9):740-48

23. Morrato EH, Druss BG, Hartung DM, et al. Small area variation and geographic and patient-specific determinants of metabolic testing in antipsychotic users. Pharmacoepidemiol Drug Saf. 2011;20(1):66-75.
24. Pringsheim T, Panagiotopoulos C, Davidson J, Ho J.; The Canadian Alliance for Monitoring Effectiveness and Safety of Antipsychotics in Children (CAMESA) guideline group. Evidence-based recommendations for monitoring safety of second-generation antipsychotics in children and youth. Paediatr Child Health. 2011;16(9):581-89. Available at: http://www. ncbi.nlm.nih.gov/pmc/articles/PMC3223902/. Accessed July 30, 2015.

25. Rifas-Shiman SL, Forman JP, Lane K, Caspard H, Gillman MW. Diabetes and lipid screening among patients in primary care: a cohort study. BMC Health Serv Res. 2008;30;8-25. Available at: http://www.biomedcentral. com/1472-6963/8/25. Accessed July 30, 2015.

26. Shi L, Ascher-Svanum H, Chiang YJ, Zhao Y, Fonseca V, Winstead D. Predictors of metabolic monitoring among schizophrenia patients with a new episode of second-generation antipsychotic use in the Veterans Health Administration. BMC Psychiatry. 2009;9:80-88. Available at: http://www. biomedcentral.com/1471-244X/9/80. Accessed July 30, 2015.

27. Copeland L, Parchman M, Zeber J, Lawrence V, Downs J, Miller A. Prediabetes assessment and follow-up in older veterans with schizophrenia. Am J Geriatr Psychiatry. 2010;18(10):887-96.

28. Moeller KE, Rigler SK, Mayorga A, Nazir N, Shireman TI. Quality of monitoring for metabolic effects associated with second generation antipsychotics in patients with schizophrenia on public insurance. Schizophr Res. 2011;126(1-3):117-23.

29. Stein BD, Leckman-Westin E, Okeke E, et al. The effects of prior authorization policies on medicaid-enrolled children's use of antipsychotic medications: evidence from two mid-Atlantic states. J Child Adolesc Psychopharmacol. 2014;24(7):374-81.

30. DeHert M, Cohen D, Bobes J, et al. Physical illness in patients with severe mental disorders. II. Barriers to care, monitoring and treatment guidelines, plus recommendations at the system and individual level. World Psychiatry. 2011;10(2):138-5l. Available at: http://www.ncbi.nlm.nih.gov/ pmc/articles/PMC3104888/. Accessed July 30, 2015.

31. Organ B, Nicholson E, Castle E. Implementing a physical health strategy in a mental health service. Australas Psychiatry. 2010;18(5):456-59.

32. Mangurian C, Giwa F, Shumway M, et al. Primary care providers' views on metabolic monitoring of outpatients taking antipsychotic medication. Psychiatr Serv. 2013;64(6):597-99. Available at: http://ps.psychiatryonline. org/doi/abs/10.1176/appi.ps.002542012?url_ver=Z39.88-2003\&rfr_ id=ori\%3Arid\%3Acrossref.org\&rfr_dat=cr_pub\%3Dpubmed. Accessed July 30, 2015.

33. Parameswaran SG, Chang C, Swenson AK, Shumway M, Olfson M, Mangurian CV. Roles in and barriers to metabolic screening for people taking antipsychotic medications: a survey of psychiatrists. Schizophr Res. 2013;143(2-3):395-96. Available at: http://www.ncbi.nlm.nih.gov/pmc/ articles/PMC3823536/. Accessed July 30, 2015. 
APPENDIX Crosswalk of Diagnostic Categories Between State Categories (DPW) to DSM-IV-TR to Research-IA Categories Used by Community Care Behavioral Health Organization

\begin{tabular}{|c|c|c|c|c|c|c|c|c|}
\hline diagn1 & Description & Subclass & MH SA & DPW_Diagcat & DSM_IV_TR & $\begin{array}{c}\text { Abuse } \\
\text { Dependency }\end{array}$ & $\begin{array}{l}\text { Alcohol } \\
\text { Drug }\end{array}$ & Research_1A \\
\hline 290 & $\begin{array}{l}\text { Senile And Presenile Organic } \\
\text { Psychotic Conditions }\end{array}$ & Organic psychotic conditions & $\mathrm{MH}$ & Organic Mental Disorders & & & & Dementia \\
\hline 290.0 & Senile Dementia Uncomp & Organic psychotic conditions & $\mathrm{MH}$ & Organic Mental Disorders & & & & Dementia \\
\hline 290.10 & Presenile Dementia & Organic psychotic conditions & $\mathrm{MH}$ & Organic Mental Disorders & & & & Dementia \\
\hline 290.11 & Presenile Delirium & Organic psychotic conditions & $\mathrm{MH}$ & Organic Mental Disorders & & & & Dementia \\
\hline 290.12 & Presenile Delusion & Organic psychotic conditions & $\mathrm{MH}$ & Organic Mental Disorders & & & & Dementia \\
\hline 290.13 & Presenile Depression & Organic psychotic conditions & MH & Organic Mental Disorders & & & & Dementia \\
\hline 290.2 & $\begin{array}{l}\text { Senile Dementia With Delusional Or } \\
\text { Depressive Features }\end{array}$ & Organic psychotic conditions & $\mathrm{MH}$ & Organic Mental Disorders & & & & Dementia \\
\hline 290.20 & Senile Delusion & Organic psychotic conditions & MH & Organic Mental Disorders & & & & Dementia \\
\hline 290.21 & Senile Depressive & Organic psychotic conditions & $\mathrm{MH}$ & Organic Mental Disorders & & & & Dementia \\
\hline 290.3 & Senile Delirium & Organic psychotic conditions & $\mathrm{MH}$ & Organic Mental Disorders & & & & Dementia \\
\hline 290.40 & Arterioscler Dement Nos & Organic psychotic conditions & $\mathrm{MH}$ & Organic Mental Disorders & $\begin{array}{l}\text { Vascular Dementia, } \\
\text { Uncomplicated }\end{array}$ & & & Dementia \\
\hline 290.41 & Arterioscler Delirium & Organic psychotic conditions & $\mathrm{MH}$ & Organic Mental Disorders & $\begin{array}{l}\text { Vascular Dementia, with } \\
\text { Delirium }\end{array}$ & & & Dementia \\
\hline 290.42 & Arterioscler Delusion & Organic psychotic conditions & $\mathrm{MH}$ & Organic Mental Disorders & $\begin{array}{l}\text { Vascular Dementia, with } \\
\text { Delusions }\end{array}$ & & & Dementia \\
\hline 290.43 & Arterioscler Depressive & Organic psychotic conditions & $\mathrm{MH}$ & Organic Mental Disorders & $\begin{array}{l}\text { Vascular Dementia, with } \\
\text { Depressed Mood }\end{array}$ & & & Dementia \\
\hline 290.8 & Senile Psychosis Nec & Organic psychotic conditions & $\mathrm{MH}$ & Organic Mental Disorders & & & & Dementia \\
\hline 290.9 & Senile Psychot Cond Nos & Organic psychotic conditions & $\mathrm{MH}$ & Organic Mental Disorders & & & & Dementia \\
\hline 291 & Alcoholic Psychoses & Organic psychotic conditions & SA & $\begin{array}{l}\text { Other Substance Related } \\
\text { Disorders }\end{array}$ & & & Alcohol & Alcohol Related Disorders \\
\hline 291.0 & Delirium Tremens & Organic psychotic conditions & SA & $\begin{array}{l}\text { Other Substance Related } \\
\text { Disorders }\end{array}$ & $\begin{array}{l}\text { Alcohol } \\
\text { [Intoxication/Withdrawal] } \\
\text { Delirium }\end{array}$ & & Alcohol & Alcohol Related Disorders \\
\hline 291.1 & Alcohol Amnestic Synd & Organic psychotic conditions & SA & $\begin{array}{l}\text { Other Substance Related } \\
\text { Disorders }\end{array}$ & $\begin{array}{l}\text { Alcohol-Induced } \\
\text { Persisting Amnestic } \\
\text { Disorder }\end{array}$ & & Alcohol & Alcohol Related Disorders \\
\hline 291.2 & Alcoholic Dementia Nec & Organic psychotic conditions & SA & $\begin{array}{l}\text { Other Substance Related } \\
\text { Disorders }\end{array}$ & $\begin{array}{l}\text { Alcohol-Induced } \\
\text { Persisting Dementia }\end{array}$ & & Alcohol & Alcohol Related Disorders \\
\hline 291.3 & Alcohol Hallucinosis & Organic psychotic conditions & SA & $\begin{array}{l}\text { Other Substance Related } \\
\text { Disorders }\end{array}$ & $\begin{array}{l}\text { Alcohol-Induced } \\
\text { Persisting Disorder, with } \\
\text { Hallucinations }\end{array}$ & & Alcohol & Alcohol Related Disorders \\
\hline 291.4 & Pathologic Alcohol Intox & Organic psychotic conditions & SA & $\begin{array}{l}\text { Other Substance Related } \\
\text { Disorders }\end{array}$ & & & Alcohol & Alcohol Related Disorders \\
\hline 291.5 & Alcoholic Jealousy & Organic psychotic conditions & SA & $\begin{array}{l}\text { Other Substance Related } \\
\text { Disorders }\end{array}$ & $\begin{array}{l}\text { Alcohol-Induced } \\
\text { Persisting Disorder, with } \\
\text { Delusions }\end{array}$ & & Alcohol & Alcohol Related Disorders \\
\hline 291.8 & Other Specified Alcoholic Psychosis & Organic psychotic conditions & SA & $\begin{array}{l}\text { Other Substance Related } \\
\text { Disorders }\end{array}$ & & & Alcohol & Alcohol Related Disorders \\
\hline
\end{tabular}


Measurement of Metabolic Monitoring in Youth and Adult Medicaid Recipients Prescribed Antipsychotics

\begin{tabular}{|c|c|c|c|c|c|c|c|c|}
\hline \multicolumn{9}{|c|}{ APPENDIX } \\
\hline diagn1 & Description & Subclass & MH SA & DPW_Diagcat & DSM_IV_TR & $\begin{array}{c}\text { Abuse } \\
\text { Dependency }\end{array}$ & $\begin{array}{l}\text { Alcohol } \\
\text { Drug }\end{array}$ & Research_1A \\
\hline 291.80 & & & & & & & & Alcohol Related Disorders \\
\hline 291.81 & Alcohol Withdrawal & Organic psychotic conditions & SA & $\begin{array}{l}\text { Other Substance Related } \\
\text { Disorders }\end{array}$ & Alcohol Withdrawal & & Alcohol & Alcohol Related Disorders \\
\hline 291.89 & Alcoholic Psychosis Nec & Organic psychotic conditions & SA & $\begin{array}{l}\text { Other Substance Related } \\
\text { Disorders }\end{array}$ & $\begin{array}{l}\text { Alcohol-Induced [Anxiety } \\
\text { Disorder/Mood } \\
\text { Disorder/Sexual } \\
\text { Dysfunction/Sleep } \\
\text { Disorder] }\end{array}$ & & Alcohol & Alcohol Related Disorders \\
\hline 291.9 & Alcoholic Psychosis Nos & Organic psychotic conditions & SA & $\begin{array}{l}\text { Other Substance Related } \\
\text { Disorders }\end{array}$ & $\begin{array}{l}\text { Alcohol-Related Disorder } \\
\text { NOS }\end{array}$ & & Alcohol & Alcohol Related Disorders \\
\hline 292 & Drug Psychoses & Organic psychotic conditions & SA & Substance Induced Disorders & & & Drug & Drug Related Disorders \\
\hline 292.0 & Drug Withdrawal Syndrome & Organic psychotic conditions & SA & Substance Induced Disorders & $\begin{array}{l}\text { [Amphetamine/Cocain/Ni } \\
\text { cotine/Opioid/Other (or } \\
\text { Unknown) } \\
\text { Substance/Sedative, } \\
\text { Hypnotic, or Anxiolytic] } \\
\text { Withdrawal }\end{array}$ & & Drug & Drug Related Disorders \\
\hline 292.1 & $\begin{array}{l}\text { Paranoid And/Or Hallucinatory } \\
\text { States Induced By Drugs }\end{array}$ & Organic psychotic conditions & SA & Substance Induced Disorders & & & Drug & Drug Related Disorders \\
\hline 292.11 & Drug Paranoid State & Organic psychotic conditions & SA & Substance Induced Disorders & $\begin{array}{l}\text { [Amphetamine/Cannabis/ } \\
\text { Cocaine/Hallucinogen/Inh } \\
\text { alant/Opioid/Other (or } \\
\text { Unknown) } \\
\text { Substance/Phencyclidine } \\
\text { /Sedative, Hypnotic, or } \\
\text { Anxiolytic]-Induced } \\
\text { Psychotic Disorder, with } \\
\text { Delusions }\end{array}$ & & Drug & Drug Related Disorders \\
\hline 292.12 & Drug Hallucinosis & Organic psychotic conditions & SA & Substance Induced Disorders & $\begin{array}{l}\text { [Amphetamine/Cannabis/ } \\
\text { Cocaine/Hallucinogen/Inh } \\
\text { alant/Opioid/Other (or } \\
\text { Unknown) } \\
\text { Substance/Phencyclidine } \\
\text { /Sedative, Hypnotic, or } \\
\text { Anxiolytic]-Induced } \\
\text { Psychotic Disorder, with } \\
\text { Hallucinations }\end{array}$ & & Drug & Drug Related Disorders \\
\hline 292.2 & Pathologic Drug Intox & Organic psychotic conditions & SA & Substance Induced Disorders & & & Drug & Drug Related Disorders \\
\hline 292.81 & Drug-Induced Delirium & Organic psychotic conditions & SA & Substance Induced Disorders & $\begin{array}{l}\text { [Amphetamine/Cannabis/ } \\
\text { Cocaine/Hallucinogen/Inh } \\
\text { alant/Opioid/Other (or } \\
\text { Unknown) } \\
\text { Substance/Phencyclidine } \\
\text { /Sedative, Hypnotic, or } \\
\text { Anxiolytic] } \\
\text { Intoxication/Withdrawal } \\
\text { Delirium }\end{array}$ & & Drug & Drug Related Disorders \\
\hline
\end{tabular}




\begin{tabular}{|c|c|c|c|c|c|c|c|c|}
\hline \multicolumn{9}{|c|}{ APPENDIX } \\
\hline diagn1 & Description & Subclass & MH SA & DPW_Diagcat & DSM_IV_TR & $\begin{array}{c}\text { Abuse } \\
\text { Dependency }\end{array}$ & $\begin{array}{l}\text { Alcohol } \\
\text { Drug }\end{array}$ & Research_1A \\
\hline 292.82 & Drug-Induced Dementia & Organic psychotic conditions & SA & Substance Induced Disorders & $\begin{array}{l}\text { [Inhalant/Other (or } \\
\text { Unknown } \\
\text { Substance/Sedative, } \\
\text { Hypnotic, or Anxiolytic]- } \\
\text { Induced Persisting } \\
\text { Dementia }\end{array}$ & & Drug & Drug Related Disorders \\
\hline 292.83 & Drug Amnestic Syndrome & Organic psychotic conditions & SA & Substance Induced Disorders & $\begin{array}{l}\text { [Other (or Unknown } \\
\text { Substance/Sedative, } \\
\text { Hypnotic, or Anxiolytic]- } \\
\text { Induced Persisting } \\
\text { Amnestic Disorder }\end{array}$ & & Drug & Drug Related Disorders \\
\hline 292.84 & Drug Depressive Syndrome & Organic psychotic conditions & SA & Substance Induced Disorders & $\begin{array}{l}\text { [Amphetamine/Cocaine/H } \\
\text { allucinogen/Inhalant/Opio } \\
\text { id/Other (or Unknown) } \\
\text { Substance/Phencyclidine } \\
\text { /Sedative, Hypnotic, or } \\
\text { Anxiolytic]-Induced Mood } \\
\text { Disorder }\end{array}$ & & Drug & Drug Related Disorders \\
\hline 292.89 & Drug Mental Disorder Nec & Organic psychotic conditions & SA & Substance Induced Disorders & $\begin{array}{l}\text { [Amphetamine/Caffeine/C } \\
\text { annabis/Cocaine/Hallucin } \\
\text { ogen/Inhalant/Opioid/Oth } \\
\text { er (or Unknown) } \\
\text { Substance/Phencyclidine } \\
\text { /Sedative, Hypnotic, or } \\
\text { Anxiolytic] [Anxiety or } \\
\text { Sleep Disorder/Sexual } \\
\text { Dysfunction/Intoxication }\end{array}$ & & Drug & Drug Related Disorders \\
\hline 292.9 & Drug Mental Disorder Nos & Organic psychotic conditions & SA & Substance Induced Disorders & $\begin{array}{l}\text { [Amphetamine/Caffeine/C } \\
\text { annabis/Cocaine/Hallucin } \\
\text { ogen/Inhalant/Nicotine/O } \\
\text { pioid/Other (or Unknown) } \\
\text { Substance/Phencyclidine } \\
\text { /Sedative, Hypnotic, or } \\
\text { Anxiolytic]-Related } \\
\text { Disorder NOS }\end{array}$ & & Drug & Drug Related Disorders \\
\hline 293 & $\begin{array}{l}\text { Transient Organic Psychotic } \\
\text { Conditions }\end{array}$ & Organic psychotic conditions & $\mathrm{MH}$ & Organic Mental Disorders & & & & Organic Mental Disorders \\
\hline 293.0 & Acute Delirium & Organic psychotic conditions & $\mathrm{MH}$ & Organic Mental Disorders & $\begin{array}{l}\text { Delirium Due to ... } \\
\text { [Indicate the General } \\
\text { Medical Condition] }\end{array}$ & & & Organic Mental Disorders \\
\hline 293.1 & Subacute Delirium & Organic psychotic conditions & $\mathrm{MH}$ & Organic Mental Disorders & & & & Organic Mental Disorders \\
\hline 293.8 & $\begin{array}{l}\text { Other Specified Transient Organic } \\
\text { Mental Disorders }\end{array}$ & Organic psychotic conditions & $\mathrm{MH}$ & Organic Mental Disorders & & & & Organic Mental Disorders \\
\hline
\end{tabular}




\begin{tabular}{|c|c|c|c|c|c|c|c|c|}
\hline \multicolumn{9}{|c|}{ APPENDIX } \\
\hline diagn1 & Description & Subclass & MH SA & DPW_Diagcat & DSM_IV_TR & $\begin{array}{c}\text { Abuse } \\
\text { Dependency }\end{array}$ & $\begin{array}{l}\text { Alcohol } \\
\text { Drug }\end{array}$ & Research $1 \mathrm{~A}$ \\
\hline 293.81 & Organic Delusional Synd & Organic psychotic conditions & MH & Organic Mental Disorders & $\begin{array}{l}\text { Psychotic Disorder Due } \\
\text { to ... [Indicate the } \\
\text { General Medical } \\
\text { Condition], With } \\
\text { Delusions }\end{array}$ & & & Organic Mental Disorders \\
\hline 293.82 & Organic Hallucinosis Syn & Organic psychotic conditions & MH & Organic Mental Disorders & $\begin{array}{l}\text { Psychotic Disorder Due } \\
\text { to ... [Indicate the } \\
\text { General Medical } \\
\text { Condition], With } \\
\text { Hallucinations }\end{array}$ & & & Organic Mental Disorders \\
\hline 293.83 & Organic Affective Synd & Organic psychotic conditions & MH & Organic Mental Disorders & $\begin{array}{l}\text { Mood Disorder Due to ... } \\
\text { [Indicate the General } \\
\text { Medical Condition] }\end{array}$ & & & Organic Mental Disorders \\
\hline 293.84 & Organic Anxiety Syndrome & Organic psychotic conditions & $\mathrm{MH}$ & Organic Mental Disorders & $\begin{array}{l}\text { Anxiety Disorder Due to } \\
\ldots \text {. Indicate the General } \\
\text { Medical Condition] }\end{array}$ & & & Organic Mental Disorders \\
\hline 293.89 & Transient Org Mental Nec & Organic psychotic conditions & MH & Organic Mental Disorders & $\begin{array}{l}\text { Catatonic Disorder Due } \\
\text { to ... [Indicate the } \\
\text { General Medical } \\
\text { Condition] }\end{array}$ & & & Organic Mental Disorders \\
\hline 293.9 & Transient Org Mental Nos & Organic psychotic conditions & $\mathrm{MH}$ & Organic Mental Disorders & $\begin{array}{l}\text { Mental Disorder NOS } \\
\text { Due to ... [Indicate the } \\
\text { General Medical } \\
\text { Condition] }\end{array}$ & & & Organic Mental Disorders \\
\hline 294 & $\begin{array}{l}\text { Other Organic Psychotic Conditions } \\
\text { (Chronic) }\end{array}$ & Organic psychotic conditions & MH & Organic Mental Disorders & & & & Dementia \\
\hline 294.0 & Amnestic Syndrome & Organic psychotic conditions & $\mathrm{MH}$ & Organic Mental Disorders & $\begin{array}{l}\text { Amnestic Disorder Due to } \\
\text {... [Indicate the General } \\
\text { Medical Condition] }\end{array}$ & & & Dementia \\
\hline 294.1 & $\begin{array}{l}\text { Dementia In Conditions Classified } \\
\text { Elsewhere }\end{array}$ & Organic psychotic conditions & MH & Organic Mental Disorders & & & & Dementia \\
\hline 294.10 & Dementia W O Behav Dist & Organic psychotic conditions & $\mathrm{MH}$ & Organic Mental Disorders & $\begin{array}{l}\text { [Dementia Due to ... } \\
\text { [Indicate the General } \\
\text { Medical Condition], } \\
\text { Without Behavioral } \\
\text { Disturbance]/[Dementia } \\
\text { of the Alzheimer's Type, } \\
\text { With [Early/Late] Onset, } \\
\text { Without Behavioral } \\
\text { Disturbance] }\end{array}$ & & & Dementia \\
\hline 294.11 & Dementia W Behavior Dist & Organic psychotic conditions & $\mathrm{MH}$ & Organic Mental Disorders & $\begin{array}{l}\text { [Dementia Due to ... } \\
\text { [Indicate the General } \\
\text { Medical Condition], With } \\
\text { Behavioral } \\
\text { Disturbance]/[Dementia } \\
\text { of the Alzheimer's Type, } \\
\text { With [Early/Late] Onset, } \\
\text { With Behavioral } \\
\text { Disturbance] }\end{array}$ & & & Dementia \\
\hline
\end{tabular}


Measurement of Metabolic Monitoring in Youth and Adult Medicaid Recipients Prescribed Antipsychotics

APPENDIX Crosswalk of Diagnostic Categories Between State Categories (DPW) to DSM-IV-TR to Research-IA Categories Used by Community Care Behavioral Health Organization (continued)

\begin{tabular}{|c|c|c|c|c|c|c|c|c|}
\hline diagn1 & Description & Subclass & MH SA & DPW_Diagcat & DSM_IV_TR & $\begin{array}{c}\text { Abuse } \\
\text { Dependency }\end{array}$ & $\begin{array}{c}\text { Alcohol } \\
\text { Drug }\end{array}$ & Research_1A \\
\hline 294.8 & Organic Brain Synd Nec & Organic psychotic conditions & $\mathrm{MH}$ & Organic Mental Disorders & $\begin{array}{l}\text { [Amnestic } \\
\text { Disorder/Dementia] NOS }\end{array}$ & & & Dementia \\
\hline 294.9 & Organic Brain Synd Nos & Organic psychotic conditions & $\mathrm{MH}$ & Organic Mental Disorders & Cognitive Disorder NOS & & & Dementia \\
\hline 295 & Schizophrenic Disorders & Other psychoses & $\mathrm{MH}$ & Schizophrenia & & & & Schizophrenia \\
\hline 295.0 & Simple Type Schizophrenia Simplex & Other psychoses & MH & Schizophrenia & & & & Schizophrenia \\
\hline 295.00 & Simpl Schizophren-Unspec & Other psychoses & $\mathrm{MH}$ & Schizophrenia & & & & Schizophrenia \\
\hline 295.01 & Simpl Schizophren-Subchr & Other psychoses & $\mathrm{MH}$ & Schizophrenia & & & & Schizophrenia \\
\hline 295.02 & Simple Schizophren-Chr & Other psychoses & $\mathrm{MH}$ & Schizophrenia & & & & Schizophrenia \\
\hline 295.03 & Simp Schiz-Subchr Exacer & Other psychoses & $\mathrm{MH}$ & Schizophrenia & & & & Schizophrenia \\
\hline 295.04 & Simpl Schizo-Chr Exacerb & Other psychoses & $\mathrm{MH}$ & Schizophrenia & & & & Schizophrenia \\
\hline 295.05 & Simpl Schizophren-Remiss & Other psychoses & $\mathrm{MH}$ & Schizophrenia & & & & Schizophrenia \\
\hline 295.1 & Disorganized Type Hebephrenia & Other psychoses & $\mathrm{MH}$ & Schizophrenia & & & & Schizophrenia \\
\hline 295.10 & Hebephrenia-Unspec & Other psychoses & MH & Schizophrenia & $\begin{array}{l}\text { Schizophrenia, } \\
\text { Disorganized Type }\end{array}$ & & & Schizophrenia \\
\hline 295.11 & Hebephrenia-Subchronic & Other psychoses & $\mathrm{MH}$ & Schizophrenia & & & & Schizophrenia \\
\hline 295.12 & Hebephrenia-Chronic & Other psychoses & $\mathrm{MH}$ & Schizophrenia & & & & Schizophrenia \\
\hline 295.13 & Hebephren-Subchr Exacerb & Other psychoses & $\mathrm{MH}$ & Schizophrenia & & & & Schizophrenia \\
\hline 295.14 & Hebephrenia-Chr Exacerb & Other psychoses & $\mathrm{MH}$ & Schizophrenia & & & & Schizophrenia \\
\hline 295.15 & Hebephrenia-Remission & Other psychoses & $\mathrm{MH}$ & Schizophrenia & & & & Schizophrenia \\
\hline 295.2 & Catatonic (Schizophrenia) & Other psychoses & $\mathrm{MH}$ & Schizophrenia & & & & Schizophrenia \\
\hline 295.20 & Catatonia-Unspec & Other psychoses & $\mathrm{MH}$ & Schizophrenia & $\begin{array}{l}\text { Schizophrenia, Catatonic } \\
\text { Type }\end{array}$ & & & Schizophrenia \\
\hline 295.21 & Catatonia-Subchronic & Other psychoses & $\mathrm{MH}$ & Schizophrenia & & & & Schizophrenia \\
\hline 295.22 & Catatonia-Chronic & Other psychoses & $\mathrm{MH}$ & Schizophrenia & & & & Schizophrenia \\
\hline 295.23 & Catatonia-Subchr Exacerb & Other psychoses & $\mathrm{MH}$ & Schizophrenia & & & & Schizophrenia \\
\hline 295.24 & Catatonia-Chr Exacerb & Other psychoses & $\mathrm{MH}$ & Schizophrenia & & & & Schizophrenia \\
\hline 295.25 & Catatonia-Remission & Other psychoses & $\mathrm{MH}$ & Schizophrenia & & & & Schizophrenia \\
\hline 295.3 & $\begin{array}{l}\text { Paranoid Type Paraphrenic } \\
\text { Schizophrenia }\end{array}$ & Other psychoses & $\mathrm{MH}$ & Schizophrenia & & & & Schizophrenia \\
\hline 295.30 & Paranoid Schizo-Unspec & Other psychoses & $\mathrm{MH}$ & Schizophrenia & $\begin{array}{l}\text { Schizophrenia, Paranoid } \\
\text { Type }\end{array}$ & & & Schizophrenia \\
\hline 295.31 & Paranoid Schizo-Subchr & Other psychoses & $\mathrm{MH}$ & Schizophrenia & & & & Schizophrenia \\
\hline 295.32 & Paranoid Schizo-Chronic & Other psychoses & $\mathrm{MH}$ & Schizophrenia & & & & Schizophrenia \\
\hline 295.33 & Paran Schizo-Subchr Exac & Other psychoses & $\mathrm{MH}$ & Schizophrenia & & & & Schizophrenia \\
\hline 295.34 & Paran Schizo-Chr Exacerb & Other psychoses & $\mathrm{MH}$ & Schizophrenia & & & & Schizophrenia \\
\hline 295.35 & Paranoid Schizo-Remiss & Other psychoses & $\mathrm{MH}$ & Schizophrenia & & & & Schizophrenia \\
\hline 295.4 & $\begin{array}{l}\text { Acute Schizophrenic Episode } \\
\text { Oneirophrenia Schizophreniform }\end{array}$ & Other psychoses & $\mathrm{MH}$ & Schizophrenia & & & & Schizophrenia \\
\hline 295.40 & Ac Schizophrenia-Unspec & Other psychoses & $\mathrm{MH}$ & Schizophrenia & $\begin{array}{l}\text { Schizophreniform } \\
\text { Disorder }\end{array}$ & & & Schizophrenia \\
\hline 295.41 & Ac Schizophrenia-Subchr & Other psychoses & $\mathrm{MH}$ & Schizophrenia & & & & Schizophrenia \\
\hline 295.42 & Ac Schizophrenia-Chr & Other psychoses & $\mathrm{MH}$ & Schizophrenia & & & & Schizophrenia \\
\hline 295.43 & Ac Schizo-Subchr Exacerb & Other psychoses & $\mathrm{MH}$ & Schizophrenia & & & & Schizophrenia \\
\hline 295.44 & Ac Schizophr-Chr Exacerb & Other psychoses & $\mathrm{MH}$ & Schizophrenia & & & & Schizophrenia \\
\hline 295.45 & Ac Schizophrenia-Remiss & Other psychoses & $\mathrm{MH}$ & Schizophrenia & & & & Schizophrenia \\
\hline 295.5 & Latent Schizophrenia & Other psychoses & $\mathrm{MH}$ & Schizophrenia & & & & Schizophrenia \\
\hline 295.50 & Latent Schizophren-Unsp & Other psychoses & $\mathrm{MH}$ & Schizophrenia & & & & Schizophrenia \\
\hline 295.51 & Lat Schizophren-Subchr & Other psychoses & $\mathrm{MH}$ & Schizophrenia & & & & Schizophrenia \\
\hline
\end{tabular}


Measurement of Metabolic Monitoring in Youth and Adult Medicaid Recipients Prescribed Antipsychotics

APPENDIX Crosswalk of Diagnostic Categories Between State Categories (DPW) to DSM-IV-TR to Research-IA Categories Used by Community Care Behavioral Health Organization (continued)

\begin{tabular}{|c|c|c|c|c|c|c|c|c|}
\hline diagn1 & Description & Subclass & MH SA & DPW_Diagcat & DSM_IV_TR & $\begin{array}{c}\text { Abuse } \\
\text { Dependency }\end{array}$ & $\begin{array}{l}\text { Alcohol } \\
\text { Drug }\end{array}$ & Research_1A \\
\hline 295.52 & Latent Schizophren-Chr & Other psychoses & $\mathrm{MH}$ & Schizophrenia & & & & Schizophrenia \\
\hline 295.53 & Lat Schizo-Subchr Exacer & Other psychoses & $\mathrm{MH}$ & Schizophrenia & & & & Schizophrenia \\
\hline 295.54 & Latent Schizo-Chr Exacer & Other psychoses & $\mathrm{MH}$ & Schizophrenia & & & & Schizophrenia \\
\hline 295.55 & Lat Schizophren-Remiss & Other psychoses & $\mathrm{MH}$ & Schizophrenia & & & & Schizophrenia \\
\hline 295.6 & Residual Schizophrenia & Other psychoses & $\mathrm{MH}$ & Schizophrenia & & & & Schizophrenia \\
\hline 295.60 & Resid Schizophren-Unsp & Other psychoses & $\mathrm{MH}$ & Schizophrenia & $\begin{array}{l}\text { Schizophrenia, Residual } \\
\text { Type }\end{array}$ & & & Schizophrenia \\
\hline 295.61 & Resid Schizophren-Subchr & Other psychoses & $\mathrm{MH}$ & Schizophrenia & & & & Schizophrenia \\
\hline 295.62 & Residual Schizophren-Chr & Other psychoses & $\mathrm{MH}$ & Schizophrenia & & & & Schizophrenia \\
\hline 295.63 & Resid Schizo-Subchr Exac & Other psychoses & $\mathrm{MH}$ & Schizophrenia & & & & Schizophrenia \\
\hline 295.64 & Resid Schizo-Chr Exacerb & Other psychoses & $\mathrm{MH}$ & Schizophrenia & & & & Schizophrenia \\
\hline 295.65 & Resid Schizophren-Remiss & Other psychoses & $\mathrm{MH}$ & Schizophrenia & & & & Schizophrenia \\
\hline 295.7 & Schizo-Affective Type & Other psychoses & $\mathrm{MH}$ & Schizophrenia & & & & Schizoaffective \\
\hline 295.70 & Schizoaffective-Unspec & Other psychoses & $\mathrm{MH}$ & Schizophrenia & Schizoaffective Disorder & & & Schizoaffective \\
\hline 295.71 & Schizoaffective-Subchr & Other psychoses & $\mathrm{MH}$ & Schizophrenia & & & & Schizoaffective \\
\hline 295.72 & Schizoaffective-Chronic & Other psychoses & $\mathrm{MH}$ & Schizophrenia & & & & Schizoaffective \\
\hline 295.73 & Schizoaff-Subchr Exacer & Other psychoses & $\mathrm{MH}$ & Schizophrenia & & & & Schizoaffective \\
\hline 295.74 & Schizoaffect-Chr Exacer & Other psychoses & $\mathrm{MH}$ & Schizophrenia & & & & Schizoaffective \\
\hline 295.75 & Schizoaffective-Remiss & Other psychoses & $\mathrm{MH}$ & Schizophrenia & & & & Schizoaffective \\
\hline 295.8 & $\begin{array}{l}\text { Other Specified Types Of } \\
\text { Schizophrenia Acute }\end{array}$ & Other psychoses & $\mathrm{MH}$ & Schizophrenia & & & & Schizophrenia \\
\hline 295.80 & Schizophrenia Nec-Unspec & Other psychoses & $\mathrm{MH}$ & Schizophrenia & & & & Schizophrenia \\
\hline 295.81 & Schizophrenia Nec-Subchr & Other psychoses & $\mathrm{MH}$ & Schizophrenia & & & & Schizophrenia \\
\hline 295.82 & Schizophrenia Nec-Chr & Other psychoses & $\mathrm{MH}$ & Schizophrenia & & & & Schizophrenia \\
\hline 295.83 & Schizo Nec-Subchr Exacer & Other psychoses & $\mathrm{MH}$ & Schizophrenia & & & & Schizophrenia \\
\hline 295.84 & Schizo Nec-Chr Exacerb & Other psychoses & $\mathrm{MH}$ & Schizophrenia & & & & Schizophrenia \\
\hline 295.85 & Schizophrenia Nec-Remiss & Other psychoses & $\mathrm{MH}$ & Schizophrenia & & & & Schizophrenia \\
\hline 295.9 & Unspecified Schizophrenia & Other psychoses & $\mathrm{MH}$ & Schizophrenia & & & & Schizophrenia \\
\hline 295.90 & Schizophrenia Nos-Unspec & Other psychoses & $\mathrm{MH}$ & Schizophrenia & $\begin{array}{l}\text { Schizophrenia, } \\
\text { Undifferentiated Type }\end{array}$ & & & Schizophrenia \\
\hline 295.91 & Schizophrenia Nos-Subchr & Other psychoses & $\mathrm{MH}$ & Schizophrenia & & & & Schizophrenia \\
\hline 295.92 & Schizophrenia Nos-Chr & Other psychoses & $\mathrm{MH}$ & Schizophrenia & & & & Schizophrenia \\
\hline 295.93 & Schizo Nos-Subchr Exacer & Other psychoses & $\mathrm{MH}$ & Schizophrenia & & & & Schizophrenia \\
\hline 295.94 & Schizo Nos-Chr Exacerb & Other psychoses & $\mathrm{MH}$ & Schizophrenia & & & & Schizophrenia \\
\hline 295.95 & Schizophrenia Nos-Remiss & Other psychoses & $\mathrm{MH}$ & Schizophrenia & & & & Schizophrenia \\
\hline 296 & Affective Psychoses & Other psychoses & $\mathrm{MH}$ & Bipolar Disorder & & & & Bipolar \\
\hline 296.0 & $\begin{array}{l}\text { Manic Disorder, Single Episode } \\
\text { Hypomania }\end{array}$ & Other psychoses & $\mathrm{MH}$ & Bipolar Disorder & & & & Bipolar \\
\hline 296.00 & Manic Disorder-Unspec & Other psychoses & $\mathrm{MH}$ & Bipolar Disorder & $\begin{array}{l}\text { Bipolar I Disorder, Single } \\
\text { Manic Episode, } \\
\text { Unspecified }\end{array}$ & & & Bipolar \\
\hline 296.01 & Manic Disorder-Mild & Other psychoses & $\mathrm{MH}$ & Bipolar Disorder & $\begin{array}{l}\text { Bipolar I Disorder, Single } \\
\text { Manic Episode, Mild }\end{array}$ & & & Bipolar \\
\hline 296.02 & Manic Disorder-Mod & Other psychoses & $\mathrm{MH}$ & Bipolar Disorder & $\begin{array}{l}\text { Bipolar I Disorder, Single } \\
\text { Manic Episode, Moderate }\end{array}$ & & & Bipolar \\
\hline
\end{tabular}




\begin{tabular}{|c|c|c|c|c|c|c|c|c|}
\hline \multicolumn{9}{|c|}{ APPENDIX } \\
\hline diagn1 & Description & Subclass & MH SA & DPW_Diagcat & DSM_IV_TR & $\begin{array}{c}\text { Abuse } \\
\text { Dependency }\end{array}$ & $\begin{array}{c}\text { Alcohol } \\
\text { Drug }\end{array}$ & Research_1A \\
\hline 296.03 & Manic Disorder-Severe & Other psychoses & $\mathrm{MH}$ & Bipolar Disorder & $\begin{array}{l}\text { Bipolar I Disorder, Single } \\
\text { Manic Episode, Severe } \\
\text { Without Psychotic } \\
\text { Features }\end{array}$ & & & Bipolar \\
\hline 296.04 & Manic Dis-Severe W Psych & Other psychoses & $\mathrm{MH}$ & Bipolar Disorder & $\begin{array}{l}\text { Bipolar I Disorder, Single } \\
\text { Manic Episode, Severe } \\
\text { With Psychotic Features }\end{array}$ & & & Bipolar \\
\hline 296.05 & Manic Dis-Partial Remiss & Other psychoses & $\mathrm{MH}$ & Bipolar Disorder & $\begin{array}{l}\text { Bipolar I Disorder, Single } \\
\text { Manic Episode, In Partial } \\
\text { Remission }\end{array}$ & & & Bipolar \\
\hline 296.06 & Manic Dis-Full Remission & Other psychoses & $\mathrm{MH}$ & Bipolar Disorder & $\begin{array}{l}\text { Bipolar I Disorder, Single } \\
\text { Manic Episode, In Full } \\
\text { Remission }\end{array}$ & & & Bipolar \\
\hline 296.1 & Manic Disorder, Recurrent Episode & Other psychoses & $\mathrm{MH}$ & Bipolar Disorder & & & & Bipolar \\
\hline 296.10 & Recur Manic Dis-Unspec & Other psychoses & $\mathrm{MH}$ & Bipolar Disorder & & & & Bipolar \\
\hline 296.11 & Recur Manic Dis-Mild & Other psychoses & $\mathrm{MH}$ & Bipolar Disorder & & & & Bipolar \\
\hline 296.12 & Recur Manic Dis-Mod & Other psychoses & $\mathrm{MH}$ & Bipolar Disorder & & & & Bipolar \\
\hline 296.13 & Recur Manic Dis-Severe & Other psychoses & $\mathrm{MH}$ & Bipolar Disorder & & & & Bipolar \\
\hline 296.14 & Recur Manic-Sev W Psycho & Other psychoses & $\mathrm{MH}$ & Bipolar Disorder & & & & Bipolar \\
\hline 296.15 & Recur Manic-Part Remiss & Other psychoses & $\mathrm{MH}$ & Bipolar Disorder & & & & Bipolar \\
\hline 296.16 & Recur Manic-Full Remiss & Other psychoses & $\mathrm{MH}$ & Bipolar Disorder & & & & Bipolar \\
\hline 296.2 & $\begin{array}{l}\text { Major Depressive Disorder, Single } \\
\text { Episode }\end{array}$ & Other psychoses & $\mathrm{MH}$ & Major Depression & & & & Major Depression \\
\hline 296.20 & Depress Psychosis-Unspec & Other psychoses & $\mathrm{MH}$ & Major Depression & $\begin{array}{l}\text { Major Depressive } \\
\text { Disorder, Single Episode, } \\
\text { Unspecified }\end{array}$ & & & Major Depression \\
\hline 296.21 & Depress Psychosis-Mild & Other psychoses & $\mathrm{MH}$ & Major Depression & $\begin{array}{l}\text { Major Depressive } \\
\text { Disorder, Single Episode, } \\
\text { Mild }\end{array}$ & & & Major Depression \\
\hline 296.22 & Depressive Psychosis-Mod & Other psychoses & $\mathrm{MH}$ & Major Depression & $\begin{array}{l}\text { Major Depressive } \\
\text { Disorder, Single Episode, } \\
\text { Moderate }\end{array}$ & & & Major Depression \\
\hline 296.23 & Depress Psychosis-Severe & Other psychoses & $\mathrm{MH}$ & Major Depression & $\begin{array}{l}\text { Major Depressive } \\
\text { Disorder, Single Episode, } \\
\text { Severe Without Psychotic } \\
\text { Features }\end{array}$ & & & Major Depression \\
\hline 296.24 & Depr Psychos-Sev W Psych & Other psychoses & $\mathrm{MH}$ & Major Depression & $\begin{array}{l}\text { Major Depressive } \\
\text { Disorder, Single Episode, } \\
\text { Severe With Psychotic } \\
\text { Features }\end{array}$ & & & Major Depression \\
\hline 296.25 & Depr Psychos-Part Remiss & Other psychoses & $\mathrm{MH}$ & Major Depression & $\begin{array}{l}\text { Major Depressive } \\
\text { Disorder, Single Episode, } \\
\text { In Partial Remission }\end{array}$ & & & Major Depression \\
\hline 296.26 & Depr Psychos-Full Remiss & Other psychoses & $\mathrm{MH}$ & Major Depression & $\begin{array}{l}\text { Major Depressive } \\
\text { Disorder, Single Episode, } \\
\text { In Full Remission }\end{array}$ & & & Major Depression \\
\hline
\end{tabular}




\begin{tabular}{|c|c|c|c|c|c|c|c|c|}
\hline \multicolumn{9}{|c|}{ APPENDIX } \\
\hline diagn1 & Description & Subclass & MH SA & DPW_Diagcat & DSM_IV_TR & $\begin{array}{c}\text { Abuse } \\
\text { Dependency }\end{array}$ & $\begin{array}{l}\text { Alcohol } \\
\text { Drug }\end{array}$ & Research_1A \\
\hline 296.3 & $\begin{array}{l}\text { Major Depressive Disorder, } \\
\text { Recurrent Episode }\end{array}$ & Other psychoses & $\mathrm{MH}$ & Major Depression & & & & Major Depression \\
\hline 296.30 & Recurr Depr Psychos-Unsp & Other psychoses & $\mathrm{MH}$ & Major Depression & $\begin{array}{l}\text { Major Depressive } \\
\text { Disorder, Recurrent, } \\
\text { Unspecified }\end{array}$ & & & Major Depression \\
\hline 296.31 & Recurr Depr Psychos-Mild & Other psychoses & $\mathrm{MH}$ & Major Depression & $\begin{array}{l}\text { Major Depressive } \\
\text { Disorder, Recurrent, Mild }\end{array}$ & & & Major Depression \\
\hline 296.32 & Recurr Depr Psychos-Mod & Other psychoses & MH & Major Depression & $\begin{array}{l}\text { Major Depressive } \\
\text { Disorder, Recurrent, } \\
\text { Moderate }\end{array}$ & & & Major Depression \\
\hline 296.33 & Recur Depr Psych-Severe & Other psychoses & $\mathrm{MH}$ & Major Depression & $\begin{array}{l}\text { Major Depressive } \\
\text { Disorder, Recurrent, } \\
\text { Severe Without Psychotic } \\
\text { Features }\end{array}$ & & & Major Depression \\
\hline 296.34 & Rec Depr Psych-Psychotic & Other psychoses & MH & Major Depression & $\begin{array}{l}\text { Major Depressive } \\
\text { Disorder, Recurrent, } \\
\text { Severe With Psychotic } \\
\text { Features }\end{array}$ & & & Major Depression \\
\hline 296.35 & Recur Depr Psyc-Part Rem & Other psychoses & MH & Major Depression & \begin{tabular}{l|} 
Major Depressive \\
Disorder, Recurrent, In \\
Partial Remission
\end{tabular} & & & Major Depression \\
\hline 296.36 & Recur Depr Psyc-Full Rem & Other psychoses & $\mathrm{MH}$ & Major Depression & $\begin{array}{l}\text { Major Depressive } \\
\text { Disorder, Recurrent, In } \\
\text { Full Remission }\end{array}$ & & & Major Depression \\
\hline 296.4 & $\begin{array}{l}\text { Bipolar Affective Disorder, Manic } \\
\text { Bipolar Disorder, Now Manic Manic- } \\
\text { Depressive Psychosis, Circular T }\end{array}$ & Other psychoses & MH & Bipolar Disorder & & & & Bipolar \\
\hline 296.40 & Bipol Aff, Manic-Unspec & Other psychoses & MH & Bipolar Disorder & $\begin{array}{l}\text { Bipolar I Disorder, Most } \\
\text { Recent Episode } \\
\text { (Hypomanic/Manic, } \\
\text { Unspecified] }\end{array}$ & & & Bipolar \\
\hline 296.41 & Bipolar Aff, Manic-Mild & Other psychoses & MH & Bipolar Disorder & $\begin{array}{l}\text { Bipolar I Disorder, Most } \\
\text { Recent Episode Manic, } \\
\text { Mild }\end{array}$ & & & Bipolar \\
\hline 296.42 & Bipolar Affec, Manic-Mod & Other psychoses & MH & Bipolar Disorder & $\begin{array}{l}\text { Bipolar I Disorder, Most } \\
\text { Recent Episode Manic, } \\
\text { Moderate }\end{array}$ & & & Bipolar \\
\hline 296.43 & Bipol Aff, Manic-Severe & Other psychoses & MH & Bipolar Disorder & $\begin{array}{l}\text { Bipolar I Disorder, Most } \\
\text { Recent Episode Manic, } \\
\text { Severe Without Psychotic } \\
\text { Features }\end{array}$ & & & Bipolar \\
\hline 296.44 & Bipol Manic-Sev W Psych & Other psychoses & MH & Bipolar Disorder & $\begin{array}{l}\text { Bipolar I Disorder, Most } \\
\text { Recent Episode Manic, } \\
\text { Severe With Psychotic } \\
\text { Features }\end{array}$ & & & Bipolar \\
\hline 296.45 & Bipol Aff Manic-Part Rem & Other psychoses & MH & Bipolar Disorder & $\begin{array}{l}\text { Bipolar I Disorder, Most } \\
\text { Recent Episode Manic, In } \\
\text { Partial Remission }\end{array}$ & & & Bipolar \\
\hline
\end{tabular}




\begin{tabular}{|c|c|c|c|c|c|c|c|c|}
\hline APF & $\begin{array}{r}\text { Crosswalk of D } \\
\text { Categories Use }\end{array}$ & $\begin{array}{l}\text { gnostic Categorie } \\
\text { by Community C }\end{array}$ & $\begin{array}{l}\text { State } \mathrm{C} \\
\text { ral He }\end{array}$ & $\begin{array}{l}\text { ategories (DPW) t } \\
\text { ath Organization }\end{array}$ & $\begin{array}{l}\text { M-IV-TR to Research-I } \\
\text { inued) }\end{array}$ & & & \\
\hline diagn1 & Description & Subclass & MH SA & DPW_Diagcat & DSM_IV_TR & $\begin{array}{c}\text { Abuse } \\
\text { Dependency }\end{array}$ & $\begin{array}{c}\text { Alcohol } \\
\text { Drug }\end{array}$ & Research_1A \\
\hline 296.46 & Bipol Aff Manic-Full Rem & Other psychoses & $\mathrm{MH}$ & Bipolar Disorder & $\begin{array}{l}\text { Bipolar I Disorder, Most } \\
\text { Recent Episode Manic, In } \\
\text { Full Remission }\end{array}$ & & & Bipolar \\
\hline 296.5 & $\begin{array}{l}\text { Bipolar Affective Disorder, } \\
\text { Depressed Bipolar Disorder, Now } \\
\text { Depressed Manic-Depressive } \\
\text { Psychosis, Ci }\end{array}$ & Other psychoses & $\mathrm{MH}$ & Bipolar Disorder & & & & Bipolar \\
\hline 296.50 & Bipolar Aff, Depr-Unspec & Other psychoses & $\mathrm{MH}$ & Bipolar Disorder & $\begin{array}{l}\text { Bipolar I Disorder, Most } \\
\text { Recent Episode } \\
\text { Depressed, Unspecified }\end{array}$ & & & Bipolar \\
\hline 296.51 & Bipolar Affec, Depr-Mild & Other psychoses & $\mathrm{MH}$ & Bipolar Disorder & $\begin{array}{l}\text { Bipolar I Disorder, Most } \\
\text { Recent Episode } \\
\text { Depressed, Mild }\end{array}$ & & & Bipolar \\
\hline 296.52 & Bipolar Affec, Depr-Mod & Other psychoses & $\mathrm{MH}$ & Bipolar Disorder & $\begin{array}{l}\text { Bipolar I Disorder, Most } \\
\text { Recent Episode } \\
\text { Depressed, Moderate }\end{array}$ & & & Bipolar \\
\hline 296.53 & Bipol Aff, Depr-Severe & Other psychoses & $\mathrm{MH}$ & Bipolar Disorder & $\begin{array}{l}\text { Bipolar I Disorder, Most } \\
\text { Recent Episode } \\
\text { Depressed, Severe } \\
\text { Without Psychotic } \\
\text { Features }\end{array}$ & & & Bipolar \\
\hline 296.54 & Bipol Depr-Sev W Psych & Other psychoses & $\mathrm{MH}$ & Bipolar Disorder & $\begin{array}{l}\text { Bipolar I Disorder, Most } \\
\text { Recent Episode } \\
\text { Depressed, Severe With } \\
\text { Psychotic Features }\end{array}$ & & & Bipolar \\
\hline 296.55 & Bipol Aff Depr-Part Rem & Other psychoses & $\mathrm{MH}$ & Bipolar Disorder & $\begin{array}{l}\text { Bipolar I Disorder, Most } \\
\text { Recent Episode } \\
\text { Depressed, In Partial } \\
\text { Remission }\end{array}$ & & & Bipolar \\
\hline 296.56 & Bipol Aff Depr-Full Rem & Other psychoses & $\mathrm{MH}$ & Bipolar Disorder & $\begin{array}{l}\text { Bipolar I Disorder, Most } \\
\text { Recent Episode } \\
\text { Depressed, In Full } \\
\text { Remission }\end{array}$ & & & Bipolar \\
\hline 296.6 & $\begin{array}{l}\text { Bipolar Affective Disorder, Mixed } \\
\text { Manic-Depressive Psychosis, } \\
\text { Circular Type, Mixed }\end{array}$ & Other psychoses & $\mathrm{MH}$ & Bipolar Disorder & & & & Bipolar \\
\hline 296.60 & Bipol Aff, Mixed-Unspec & Other psychoses & $\mathrm{MH}$ & Bipolar Disorder & $\begin{array}{l}\text { Bipolar I Disorder, Most } \\
\text { Recent Episode Mixed, } \\
\text { Unspecified }\end{array}$ & & & Bipolar \\
\hline 296.61 & Bipolar Aff, Mixed-Mild & Other psychoses & MH & Bipolar Disorder & $\begin{array}{l}\text { Bipolar I Disorder, Most } \\
\text { Recent Episode Mixed, } \\
\text { Mild }\end{array}$ & & & Bipolar \\
\hline 296.62 & Bipolar Affec, Mixed-Mod & Other psychoses & $\mathrm{MH}$ & Bipolar Disorder & $\begin{array}{l}\text { Bipolar I Disorder, Most } \\
\text { Recent Episode Mixed, } \\
\text { Moderate }\end{array}$ & & & Bipolar \\
\hline 296.63 & Bipol Aff, Mixed-Severe & Other psychoses & $\mathrm{MH}$ & Bipolar Disorder & $\begin{array}{l}\text { Bipolar I Disorder, Most } \\
\text { Recent Episode Mixed, } \\
\text { Severe Without Psychotic } \\
\text { Features }\end{array}$ & & & Bipolar \\
\hline
\end{tabular}




\begin{tabular}{|c|c|c|c|c|c|c|c|c|}
\hline \multicolumn{9}{|c|}{ APPENDIX } \\
\hline diagn1 & Description & Subclass & MH SA & DPW_Diagcat & DSM_IV_TR & \begin{tabular}{|c|} 
Abuse \\
Dependency \\
\end{tabular} & $\begin{array}{l}\text { Alcohol } \\
\text { Drug }\end{array}$ & Research_1A \\
\hline 296.64 & Bipol Mixed-Sev W Psych & Other psychoses & $\mathrm{MH}$ & Bipolar Disorder & $\begin{array}{l}\text { Bipolar I Disorder, Most } \\
\text { Recent Episode Mixed, } \\
\text { Severe With Psychotic } \\
\text { Features }\end{array}$ & & & Bipolar \\
\hline 296.65 & Bipol Aff, Mix-Part Rem & Other psychoses & $\mathrm{MH}$ & Bipolar Disorder & $\begin{array}{l}\text { Bipolar I Disorder, Most } \\
\text { Recent Episode Mixed, In } \\
\text { Partial Remission }\end{array}$ & & & Bipolar \\
\hline 296.66 & Bipol Aff, Mix-Full Rem & Other psychoses & $\mathrm{MH}$ & Bipolar Disorder & $\begin{array}{l}\text { Bipolar I Disorder, Most } \\
\text { Recent Episode Mixed, In } \\
\text { Full Remission }\end{array}$ & & & Bipolar \\
\hline 296.7 & Bipolar Affective Nos & Other psychoses & $\mathrm{MH}$ & Bipolar Disorder & $\begin{array}{l}\text { Bipolar I Disorder, Most } \\
\text { Recent Episode } \\
\text { Unspecified }\end{array}$ & & & Bipolar \\
\hline 296.70 & $\begin{array}{l}\text { Invalid Code - } 296.7=\text { Bipolar } \\
\text { Affective Nos }\end{array}$ & Other psychoses & $\mathrm{MH}$ & Bipolar Disorder & & & & Bipolar \\
\hline 296.8 & $\begin{array}{l}\text { Manic-Depressive Psychosis, Other } \\
\text { And Unspecified }\end{array}$ & Other psychoses & MH & Bipolar Disorder & & & & Bipolar \\
\hline 296.80 & Manic-Depressive Nos & Other psychoses & $\mathrm{MH}$ & Bipolar Disorder & Bipolar Disorder NOS & & & Bipolar \\
\hline 296.81 & Atypical Manic Disorder & Other psychoses & $\mathrm{MH}$ & Bipolar Disorder & & & & Bipolar \\
\hline 296.82 & Atypical Depressive Dis & Other psychoses & $\mathrm{MH}$ & Bipolar Disorder & & & & Bipolar \\
\hline 296.89 & Manic-Depressive Nec & Other psychoses & $\mathrm{MH}$ & Bipolar Disorder & Bipolar II Disorder & & & Bipolar \\
\hline 296.9 & $\begin{array}{l}\text { Other And Unspecified Affective } \\
\text { Psychoses }\end{array}$ & Other psychoses & $\mathrm{MH}$ & Major Depression & Diputar in Lisutuer & & & $\begin{array}{l}\text { Dysthimia/Depression } \\
\text { NOS }\end{array}$ \\
\hline 296.90 & Affective Psychosis Nos & Other psychoses & $\mathrm{MH}$ & Major Depression & Mood Disorder NOS & & & $\begin{array}{l}\text { Dysthimia/Depression } \\
\text { NOS }\end{array}$ \\
\hline 296.99 & Affective Psychoses Nec & Other psychoses & MH & Major Depression & & & & $\begin{array}{l}\text { Dysthimia/Depression } \\
\text { NOS }\end{array}$ \\
\hline 297 & Paranoid States & Other psychoses & $\mathrm{MH}$ & Paranoid States & & & & Paranoid States \\
\hline 297.0 & Paranoid State, Simple & Other psychoses & $\mathrm{MH}$ & Paranoid States & & & & Paranoid States \\
\hline 297.1 & Paranoia & Other psychoses & $\mathrm{MH}$ & Paranoid States & Delusional Disorder & & & Paranoid States \\
\hline 297.2 & Paraphrenia & Other psychoses & $\mathrm{MH}$ & Paranoid States & & & & Paranoid States \\
\hline 297.3 & Shared Paranoid Disorder & Other psychoses & MH & Paranoid States & $\begin{array}{l}\text { Shared Psychotic } \\
\text { Disorder }\end{array}$ & & & Paranoid States \\
\hline 297.8 & Paranoid States Nec & Other psychoses & $\mathrm{MH}$ & Paranoid States & & & & Paranoid States \\
\hline 297.9 & Paranoid State Nos & Other psychoses & $\mathrm{MH}$ & Paranoid States & & & & Paranoid States \\
\hline 298 & Other Nonorganic Psychoses & Other psychoses & MH & Other Nonorganic Psychoses & & & & $\begin{array}{l}\text { Other Nonorganic } \\
\text { Psychoses }\end{array}$ \\
\hline 298.0 & React Depress Psychosis & Other psychoses & $\mathrm{MH}$ & Other Nonorganic Psychoses & & & & $\begin{array}{l}\text { Other Nonorganic } \\
\text { Psychoses }\end{array}$ \\
\hline 298.1 & Excitativ Type Psychosis & Other psychoses & MH & Other Nonorganic Psychoses & & & & $\begin{array}{l}\text { Other Nonorganic } \\
\text { Psychoses }\end{array}$ \\
\hline 298.2 & Reactive Confusion & Other psychoses & $\mathrm{MH}$ & Other Nonorganic Psychoses & & & & $\begin{array}{l}\text { Other Nonorganic } \\
\text { Psychoses }\end{array}$ \\
\hline 298.3 & Acute Paranoid Reaction & Other psychoses & MH & Other Nonorganic Psychoses & & & & $\begin{array}{l}\text { Other Nonorganic } \\
\text { Psychoses }\end{array}$ \\
\hline 298.4 & Psychogen Paranoid Psych & Other psychoses & $\mathrm{MH}$ & Other Nonorganic Psychoses & & & & $\begin{array}{l}\text { Other Nonorganic } \\
\text { Psychoses }\end{array}$ \\
\hline 298.8 & React Psychosis Nec Nos & Other psychoses & $\mathrm{MH}$ & Other Nonorganic Psychoses & Brief Psychotic Disorder & & & $\begin{array}{l}\text { Other Nonorganic } \\
\text { Psychoses }\end{array}$ \\
\hline
\end{tabular}




\begin{tabular}{|c|c|c|c|c|c|c|c|c|}
\hline \multicolumn{9}{|c|}{ APPENDIX } \\
\hline diagn1 & Description & Subclass & MH SA & DPW_Diagcat & DSM_IV_TR & $\begin{array}{c}\text { Abuse } \\
\text { Dependency }\end{array}$ & $\begin{array}{c}\text { Alcohol } \\
\text { Drug }\end{array}$ & Research_1A \\
\hline 298.80 & $\begin{array}{l}\text { Invalid Code - } 298.8=\text { React } \\
\text { Psychosis Nec Nos }\end{array}$ & Other psychoses & $\mathrm{MH}$ & Other Nonorganic Psychoses & & & & $\begin{array}{l}\text { Other Nonorganic } \\
\text { Psychoses }\end{array}$ \\
\hline 298.9 & Psychosis Nos & Other psychoses & $\mathrm{MH}$ & Unspecified Psychoses & Psychotic Disorder NOS & & & $\begin{array}{l}\text { Other Nonorganic } \\
\text { Psychoses }\end{array}$ \\
\hline 298.90 & $\begin{array}{l}\text { Invalid Code - } 298.9 \text { = Psychosis } \\
\text { Nos }\end{array}$ & Other psychoses & $\mathrm{MH}$ & Unspecified Psychoses & & & & $\begin{array}{l}\text { Other Nonorganic } \\
\text { Psychoses }\end{array}$ \\
\hline 299 & $\begin{array}{l}\text { Psychoses With Origin Specific To } \\
\text { Childhood }\end{array}$ & Other psychoses & $\mathrm{MH}$ & Psychosis w/Childhood Origin & & & & Autism \\
\hline 299.0 & Infantile Autism & Other psychoses & $\mathrm{MH}$ & Psychosis w/Childhood Origin & & & & Autism \\
\hline 299.00 & Infantile Autism-Active & Other psychoses & $\mathrm{MH}$ & Psychosis w/Childhood Origin & Autistic Disorder & & & Autism \\
\hline 299.01 & Infantile Autism-Resid & Other psychoses & $\mathrm{MH}$ & Psychosis w/Childhood Origin & & & & Autism \\
\hline 299.1 & $\begin{array}{l}\text { Disintegrative Psychosis (Heller'S } \\
\text { Syndrome) }\end{array}$ & Other psychoses & $\mathrm{MH}$ & Psychosis w/Childhood Origin & & & & Autism \\
\hline 299.10 & Disintegr Psych-Active & Other psychoses & $\mathrm{MH}$ & Psychosis w/Childhood Origin & $\begin{array}{l}\text { Childhood Disintegrative } \\
\text { Disorder }\end{array}$ & & & Autism \\
\hline 299.11 & Disintegr Psych-Residual & Other psychoses & $\mathrm{MH}$ & Psychosis w/Childhood Origin & & & & Autism \\
\hline 299.8 & $\begin{array}{l}\text { Other Specified Early Childhood } \\
\text { Psychoses }\end{array}$ & Other psychoses & $\mathrm{MH}$ & Psychosis w/Childhood Origin & & & & Autism \\
\hline 299.80 & Child Psychos Nec-Active & Other psychoses & $\mathrm{MH}$ & Psychosis w/Childhood Origin & $\begin{array}{l}\text { [Asperger's } \\
\text { Disorder/Pervasive } \\
\text { Developmental Disorder } \\
\text { NOS/Rett's Disorder] }\end{array}$ & & & Autism \\
\hline 299.81 & Child Psychos Nec-Resid & Other psychoses & $\mathrm{MH}$ & Psychosis w/Childhood Origin & & & & Autism \\
\hline 299.9 & $\begin{array}{l}\text { Schizophrenic Syndrome Of } \\
\text { Childhood Nos }\end{array}$ & Other psychoses & $\mathrm{MH}$ & Psychosis w/Childhood Origin & & & & Autism \\
\hline 299.90 & Child Psychos Nos-Active & Other psychoses & $\mathrm{MH}$ & Psychosis w/Childhood Origin & & & & Autism \\
\hline 299.91 & Child Psychos Nos-Resid & Other psychoses & $\mathrm{MH}$ & Psychosis w/Childhood Origin & & & & Autism \\
\hline 300 & Neurotic Disorders & $\begin{array}{l}\text { Neurotic disorders, personality } \\
\text { disorders, and oth }\end{array}$ & $\mathrm{MH}$ & Neurotic Disorders & & & & Anxiety \\
\hline 300.0 & Anxiety States & $\begin{array}{l}\text { Neurotic disorders, personality } \\
\text { disorders, and oth }\end{array}$ & $\mathrm{MH}$ & Neurotic Disorders & & & & Anxiety \\
\hline 300.00 & Anxiety State Nos & $\begin{array}{l}\text { Neurotic disorders, personality } \\
\text { disorders, and oth }\end{array}$ & $\mathrm{MH}$ & Neurotic Disorders & Anxiety Disorder NOS & & & Anxiety \\
\hline 300.01 & Panic Disorder & $\begin{array}{l}\text { Neurotic disorders, personality } \\
\text { disorders, and oth }\end{array}$ & $\mathrm{MH}$ & Neurotic Disorders & $\begin{array}{l}\text { Panic Disorder without } \\
\text { Agoraphobia }\end{array}$ & & & Anxiety \\
\hline 300.02 & Generalized Anxiety Dis & $\begin{array}{l}\text { Neurotic disorders, personality } \\
\text { disorders, and oth }\end{array}$ & MH & Neurotic Disorders & $\begin{array}{l}\text { Generalized Anxiety } \\
\text { Disorder }\end{array}$ & & & Anxiety \\
\hline 300.09 & Anxiety State $\mathrm{Nec}$ & $\begin{array}{l}\text { Neurotic disorders, personality } \\
\text { disorders, and oth }\end{array}$ & $\mathrm{MH}$ & Neurotic Disorders & & & & Anxiety \\
\hline 300.1 & Hysteria & $\begin{array}{l}\text { Neurotic disorders, personality } \\
\text { disorders, and oth }\end{array}$ & $\mathrm{MH}$ & Neurotic Disorders & & & & Somatoform \\
\hline 300.10 & Hysteria Nos & $\begin{array}{l}\text { Neurotic disorders, personality } \\
\text { disorders, and oth }\end{array}$ & MH & Neurotic Disorders & & & & Somatoform \\
\hline
\end{tabular}


Measurement of Metabolic Monitoring in Youth and Adult Medicaid Recipients Prescribed Antipsychotics

\begin{tabular}{|c|c|c|c|c|c|c|c|c|}
\hline \multicolumn{9}{|c|}{ APPENDIX } \\
\hline diagn1 & Description & Subclass & MH SA & DPW_Diagcat & DSM_IV_TR & \begin{tabular}{|c|} 
Abuse \\
Dependency \\
\end{tabular} & $\begin{array}{l}\text { Alcohol } \\
\text { Drug }\end{array}$ & Research_1A \\
\hline 300.11 & Conversion Disorder & $\begin{array}{l}\text { Neurotic disorders, personality } \\
\text { disorders, and oth }\end{array}$ & $\mathrm{MH}$ & Neurotic Disorders & Conversion Disorder & & & Somatoform \\
\hline 300.12 & Psychogenic Amnesia & $\begin{array}{l}\text { Neurotic disorders, personality } \\
\text { disorders, and oth }\end{array}$ & $\mathrm{MH}$ & Neurotic Disorders & Dissociative Amnesia & & & Dissociative Disorders \\
\hline 300.13 & Psychogenic Fugue & $\begin{array}{l}\text { Neurotic disorders, personality } \\
\text { disorders, and oth }\end{array}$ & $\mathrm{MH}$ & Neurotic Disorders & Dissociative Fugue & & & Dissociative Disorders \\
\hline 300.14 & Multiple Personality & $\begin{array}{l}\text { Neurotic disorders, personality } \\
\text { disorders, and oth }\end{array}$ & $\mathrm{MH}$ & Neurotic Disorders & $\begin{array}{l}\text { Dissociative Identity } \\
\text { Disorder }\end{array}$ & & & Dissociative Disorders \\
\hline 300.15 & Dissociative React Nos & $\begin{array}{l}\text { Neurotic disorders, personality } \\
\text { disorders, and oth }\end{array}$ & $\mathrm{MH}$ & Neurotic Disorders & $\begin{array}{l}\text { Dissociative Disorder } \\
\text { NOS }\end{array}$ & & & Dissociative Disorders \\
\hline 300.16 & Factitious III W Symptom & $\begin{array}{l}\text { Neurotic disorders, personality } \\
\text { disorders, and oth }\end{array}$ & $\mathrm{MH}$ & Neurotic Disorders & $\begin{array}{l}\text { Factitious Disorder with } \\
\text { Predominantly } \\
\text { Psychological Signs and } \\
\text { Symptoms }\end{array}$ & & & Factitious \\
\hline 300.19 & Factitious III Nec Nos & $\begin{array}{l}\text { Neurotic disorders, personality } \\
\text { disorders, and oth }\end{array}$ & MH & Neurotic Disorders & $\begin{array}{l}\text { Factitious Disorder } \\
\text { [NOS/with Combined } \\
\text { Psychological and } \\
\text { Physical Signs and } \\
\text { Symptoms/with } \\
\text { Predominantly Physical } \\
\text { Signs and Symptoms] }\end{array}$ & & & Factitious \\
\hline 300.2 & Phobic Disorders & $\begin{array}{l}\text { Neurotic disorders, personality } \\
\text { disorders, and oth }\end{array}$ & $\mathrm{MH}$ & Neurotic Disorders & & & & Anxiety \\
\hline 300.20 & Phobia Nos & $\begin{array}{l}\text { Neurotic disorders, personality } \\
\text { disorders, and oth }\end{array}$ & MH & Neurotic Disorders & & & & Anxiety \\
\hline 300.21 & Agoraphobia With Panic & $\begin{array}{l}\text { Neurotic disorders, personality } \\
\text { disorders, and oth }\end{array}$ & MH & Neurotic Disorders & $\begin{array}{l}\text { Panic Disorder with } \\
\text { Agoraphobia }\end{array}$ & & & Anxiety \\
\hline 300.22 & Agoraphobia W O Panic & $\begin{array}{l}\text { Neurotic disorders, personality } \\
\text { disorders, and oth }\end{array}$ & $\mathrm{MH}$ & Neurotic Disorders & $\begin{array}{l}\text { Agoraphobia without } \\
\text { History of Panic Disorder }\end{array}$ & & & Anxiety \\
\hline 300.23 & Social Phobia & $\begin{array}{l}\text { Neurotic disorders, personality } \\
\text { disorders, and oth }\end{array}$ & $\mathrm{MH}$ & Neurotic Disorders & Social Phobia & & & Anxiety \\
\hline 300.29 & Isolated Phobias Nec & $\begin{array}{l}\text { Neurotic disorders, personality } \\
\text { disorders, and oth }\end{array}$ & $\mathrm{MH}$ & Neurotic Disorders & Specific Phobia & & & Anxiety \\
\hline 300.3 & Obsessive-Compulsive Dis & $\begin{array}{l}\text { Neurotic disorders, personality } \\
\text { disorders, and oth }\end{array}$ & $\mathrm{MH}$ & Neurotic Disorders & $\begin{array}{l}\text { Obsessive-Compulsive } \\
\text { Disorder }\end{array}$ & & & Anxiety \\
\hline 300.4 & $\begin{array}{l}\text { Neurotic Depression-Dysthymic } \\
\text { Disorder }\end{array}$ & $\begin{array}{l}\text { Neurotic disorders, personality } \\
\text { disorders, and oth }\end{array}$ & $\mathrm{MH}$ & Neurotic Disorders & Dysthymic Disorder & & & $\begin{array}{l}\text { Dysthimia/Depression } \\
\text { NOS }\end{array}$ \\
\hline 300.40 & $\begin{array}{l}\text { Invalid code - } 300.4=\text { Neurotic } \\
\text { Depression-Dysthymic Disorder }\end{array}$ & $\begin{array}{l}\text { Neurotic disorders, personality } \\
\text { disorders, and oth }\end{array}$ & $\mathrm{MH}$ & Neurotic Disorders & & & & $\begin{array}{l}\text { Dysthimia/Depression } \\
\text { NOS }\end{array}$ \\
\hline 300.5 & Neurasthenia & $\begin{array}{l}\text { Neurotic disorders, personality } \\
\text { disorders, and oth }\end{array}$ & MH & Neurotic Disorders & & & & $\begin{array}{l}\text { Dysthimia/Depression } \\
\text { NOS }\end{array}$ \\
\hline 300.6 & Depersonalization Synd & $\begin{array}{l}\text { Neurotic disorders, personality } \\
\text { disorders, and oth }\end{array}$ & $\mathrm{MH}$ & Neurotic Disorders & $\begin{array}{l}\text { Depersonalization } \\
\text { Disorder }\end{array}$ & & & Dissociative Disorders \\
\hline 300.7 & Hypochondriasis & $\begin{array}{l}\text { Neurotic disorders, personality } \\
\text { disorders, and oth }\end{array}$ & MH & Neurotic Disorders & $\begin{array}{l}\text { [Body Dysmorphic } \\
\text { Disorder/Hypochondriasi } \\
\text { s] }\end{array}$ & & & Somatoform \\
\hline 300.8 & Other Neurotic Disorders & $\begin{array}{l}\text { Neurotic disorders, personality } \\
\text { disorders, and oth }\end{array}$ & $\mathrm{MH}$ & Neurotic Disorders & & & & Anxiety \\
\hline
\end{tabular}




\begin{tabular}{|c|c|c|c|c|c|c|c|c|}
\hline \begin{tabular}{|l} 
diagn1 \\
\end{tabular} & Description & Subclass & MH SA & DPW_Diagcat & DSM_IV_TR & $\begin{array}{c}\text { Abuse } \\
\text { Dependency }\end{array}$ & $\begin{array}{c}\text { Alcohol } \\
\text { Drug }\end{array}$ & Research_1A \\
\hline 300.81 & Somatization Disorder & $\begin{array}{l}\text { Neurotic disorders, personality } \\
\text { disorders, and oth }\end{array}$ & $\mathrm{MH}$ & Neurotic Disorders & Somatization Disorder & & & Somatoform \\
\hline 300.82 & Undiff Somatoform Disrdr & $\begin{array}{l}\text { Neurotic disorders, personality } \\
\text { disorders, and oth }\end{array}$ & $\mathrm{MH}$ & Neurotic Disorders & $\begin{array}{l}\text { [Somatoform Disorder } \\
\text { NOS/Undifferentiated } \\
\text { Somatoform Disorder] }\end{array}$ & & & Somatoform \\
\hline 300.89 & Neurotic Disorders Nec & $\begin{array}{l}\text { Neurotic disorders, personality } \\
\text { disorders, and oth }\end{array}$ & $\mathrm{MH}$ & Neurotic Disorders & & & & Anxiety \\
\hline 300.9 & Neurotic Disorder Nos & $\begin{array}{l}\text { Neurotic disorders, personality } \\
\text { disorders, and oth }\end{array}$ & $\mathrm{MH}$ & Neurotic Disorders & $\begin{array}{l}\text { Unspecified Mental } \\
\text { Disorder (nonpsychotic) }\end{array}$ & & & Anxiety \\
\hline 300.99 & UNKNOWN & UNKNOWN & $\mathrm{MH}$ & Neurotic Disorders & & & & Anxiety \\
\hline 301 & Personality Disorders & $\begin{array}{l}\text { Neurotic disorders, personality } \\
\text { disorders, and oth }\end{array}$ & $\mathrm{MH}$ & $\begin{array}{l}\text { Other Neurotic and } \\
\text { Nonpsychotic Mental } \\
\text { Disorders }\end{array}$ & & & & Personality \\
\hline 301.0 & Paranoid Personality & $\begin{array}{l}\text { Neurotic disorders, personality } \\
\text { disorders, and oth }\end{array}$ & $\mathrm{MH}$ & $\begin{array}{l}\text { Other Neurotic and } \\
\text { Nonpsychotic Mental } \\
\text { Disorders }\end{array}$ & $\begin{array}{l}\text { Paranoid Personality } \\
\text { Disorder }\end{array}$ & & & Personality \\
\hline 301.1 & Affective Personality Disorder & $\begin{array}{l}\text { Neurotic disorders, personality } \\
\text { disorders, and oth }\end{array}$ & $\mathrm{MH}$ & $\begin{array}{l}\text { Other Neurotic and } \\
\text { Nonpsychotic Mental } \\
\text { Disorders }\end{array}$ & & & & Personality \\
\hline 301.10 & Affectiv Personality Nos & $\begin{array}{l}\text { Neurotic disorders, personality } \\
\text { disorders, and oth }\end{array}$ & $\mathrm{MH}$ & $\begin{array}{l}\text { Other Neurotic and } \\
\text { Nonpsychotic Mental } \\
\text { Disorders }\end{array}$ & & & & Personality \\
\hline 301.11 & Chronic Hypomanic Person & $\begin{array}{l}\text { Neurotic disorders, personality } \\
\text { disorders, and oth }\end{array}$ & $\mathrm{MH}$ & $\begin{array}{l}\text { Other Neurotic and } \\
\text { Nonpsychotic Mental } \\
\text { Disorders }\end{array}$ & & & & Personality \\
\hline 301.12 & Chr Depressive Person & $\begin{array}{l}\text { Neurotic disorders, personality } \\
\text { disorders, and oth }\end{array}$ & $\mathrm{MH}$ & $\begin{array}{l}\text { Other Neurotic and } \\
\text { Nonpsychotic Mental } \\
\text { Disorders }\end{array}$ & & & & Personality \\
\hline 301.13 & Cyclothymic Disorder & $\begin{array}{l}\text { Neurotic disorders, personality } \\
\text { disorders, and oth }\end{array}$ & $\mathrm{MH}$ & $\begin{array}{l}\text { Other Neurotic and } \\
\text { Nonpsychotic Mental } \\
\text { Disorders }\end{array}$ & Cyclothymic Disorder & & & Bipolar \\
\hline 301.2 & Schizoid Personality Disorder & $\begin{array}{l}\text { Neurotic disorders, personality } \\
\text { disorders, and oth }\end{array}$ & $\mathrm{MH}$ & $\begin{array}{l}\text { Other Neurotic and } \\
\text { Nonpsychotic Mental } \\
\text { Disorders }\end{array}$ & & & & Personality \\
\hline 301.20 & Schizoid Personality Nos & $\begin{array}{l}\text { Neurotic disorders, personality } \\
\text { disorders, and oth }\end{array}$ & $\mathrm{MH}$ & $\begin{array}{l}\text { Other Neurotic and } \\
\text { Nonpsychotic Mental } \\
\text { Disorders }\end{array}$ & $\begin{array}{l}\text { Schizoid Personality } \\
\text { Disorder }\end{array}$ & & & Personality \\
\hline 301.21 & Introverted Personality & $\begin{array}{l}\text { Neurotic disorders, personality } \\
\text { disorders, and oth }\end{array}$ & $\mathrm{MH}$ & $\begin{array}{l}\text { Other Neurotic and } \\
\text { Nonpsychotic Mental } \\
\text { Disorders }\end{array}$ & & & & Personality \\
\hline 301.22 & Schizotypal Personality & $\begin{array}{l}\text { Neurotic disorders, personality } \\
\text { disorders, and oth }\end{array}$ & $\mathrm{MH}$ & $\begin{array}{l}\text { Other Neurotic and } \\
\text { Nonpsychotic Mental } \\
\text { Disorders }\end{array}$ & $\begin{array}{l}\text { Schizotypal Personality } \\
\text { Disorder }\end{array}$ & & & Personality \\
\hline 301.3 & Explosive Personality & $\begin{array}{l}\text { Neurotic disorders, personality } \\
\text { disorders, and oth }\end{array}$ & $\mathrm{MH}$ & $\begin{array}{l}\text { Other Neurotic and } \\
\text { Nonpsychotic Mental } \\
\text { Disorders }\end{array}$ & & & & Personality \\
\hline 301.4 & Compulsive Personality & $\begin{array}{l}\text { Neurotic disorders, personality } \\
\text { disorders, and oth }\end{array}$ & $\mathrm{MH}$ & $\begin{array}{l}\text { Other Neurotic and } \\
\text { Nonpsychotic Mental } \\
\text { Disorders }\end{array}$ & $\begin{array}{l}\text { Obsessive-Compulsive } \\
\text { Personality Disorder }\end{array}$ & & & Personality \\
\hline
\end{tabular}




\begin{tabular}{|c|c|c|c|c|c|c|c|c|}
\hline \multicolumn{9}{|c|}{ APPENDIX } \\
\hline diagn1 & Description & Subclass & MH SA & DPW_Diagcat & DSM_IV_TR & \begin{tabular}{c|} 
Abuse \\
Dependency
\end{tabular} & $\begin{array}{l}\text { Alcohol } \\
\text { Drug }\end{array}$ & Research_1A \\
\hline 301.5 & Histrionic Personality Disorder & $\begin{array}{l}\text { Neurotic disorders, personality } \\
\text { disorders, and oth }\end{array}$ & MH & $\begin{array}{l}\text { Other Neurotic and } \\
\text { Nonpsychotic Mental } \\
\text { Disorders }\end{array}$ & & & & Personality \\
\hline 301.50 & Histrionic Person Nos & $\begin{array}{l}\text { Neurotic disorders, personality } \\
\text { disorders, and oth }\end{array}$ & $\mathrm{MH}$ & $\begin{array}{l}\text { Other Neurotic and } \\
\text { Nonpsychotic Mental } \\
\text { Disorders }\end{array}$ & $\begin{array}{l}\text { Histrionic Personality } \\
\text { Disorder }\end{array}$ & & & Personality \\
\hline 301.51 & Chr Factitious Illness & $\begin{array}{l}\text { Neurotic disorders, personality } \\
\text { disorders, and oth }\end{array}$ & $\mathrm{MH}$ & $\begin{array}{l}\text { Other Neurotic and } \\
\text { Nonpsychotic Mental } \\
\text { Disorders }\end{array}$ & & & & Factitious \\
\hline 301.59 & Histrionic Person Nec & $\begin{array}{l}\text { Neurotic disorders, personality } \\
\text { disorders, and oth }\end{array}$ & MH & $\begin{array}{l}\text { Other Neurotic and } \\
\text { Nonpsychotic Mental } \\
\text { Disorders }\end{array}$ & & & & Personality \\
\hline 301.6 & Dependent Personality & $\begin{array}{l}\text { Neurotic disorders, personality } \\
\text { disorders, and oth }\end{array}$ & MH & $\begin{array}{l}\text { Other Neurotic and } \\
\text { Nonpsychotic Mental } \\
\text { Disorders }\end{array}$ & $\begin{array}{l}\text { Dependent Personality } \\
\text { Disorder }\end{array}$ & & & Personality \\
\hline 301.7 & Antisocial Personality & $\begin{array}{l}\text { Neurotic disorders, personality } \\
\text { disorders, and oth }\end{array}$ & MH & $\begin{array}{l}\text { Other Neurotic and } \\
\text { Nonpsychotic Mental } \\
\text { Disorders }\end{array}$ & $\begin{array}{l}\text { Antisocial Personality } \\
\text { Disorder }\end{array}$ & & & Personality \\
\hline 301.8 & Other Personality Disorders & $\begin{array}{l}\text { Neurotic disorders, personality } \\
\text { disorders, and oth }\end{array}$ & MH & $\begin{array}{l}\text { Other Neurotic and } \\
\text { Nonpsychotic Mental } \\
\text { Disorders }\end{array}$ & & & & Personality \\
\hline 301.81 & Narcissistic Personality & $\begin{array}{l}\text { Neurotic disorders, personality } \\
\text { disorders, and oth }\end{array}$ & $\mathrm{MH}$ & $\begin{array}{l}\text { Other Neurotic and } \\
\text { Nonpsychotic Mental } \\
\text { Disorders }\end{array}$ & $\begin{array}{l}\text { Narcissistic Personality } \\
\text { Disorder }\end{array}$ & & & Personality \\
\hline 301.82 & Avoidant Personality & $\begin{array}{l}\text { Neurotic disorders, personality } \\
\text { disorders, and oth }\end{array}$ & MH & $\begin{array}{l}\text { Other Neurotic and } \\
\text { Nonpsychotic Mental } \\
\text { Disorders }\end{array}$ & $\begin{array}{l}\text { Avoidant Personality } \\
\text { Disorder }\end{array}$ & & & Personality \\
\hline 301.83 & Borderline Personality & $\begin{array}{l}\text { Neurotic disorders, personality } \\
\text { disorders, and oth }\end{array}$ & $\mathrm{MH}$ & $\begin{array}{l}\text { Borderline Personality } \\
\text { Disorder }\end{array}$ & $\begin{array}{l}\text { Borderline Personality } \\
\text { Disorder }\end{array}$ & & & Personality \\
\hline 301.84 & Passive-Aggressiv Person & $\begin{array}{l}\text { Neurotic disorders, personality } \\
\text { disorders, and oth }\end{array}$ & MH & $\begin{array}{l}\text { Other Neurotic and } \\
\text { Nonpsychotic Mental } \\
\text { Disorders }\end{array}$ & & & & Personality \\
\hline 301.89 & Personality Disorder $\mathrm{Nec}$ & $\begin{array}{l}\text { Neurotic disorders, personality } \\
\text { disorders, and oth }\end{array}$ & MH & $\begin{array}{l}\text { Other Neurotic and } \\
\text { Nonpsychotic Mental } \\
\text { Disorders }\end{array}$ & & & & Personality \\
\hline 301.9 & Personality Disorder Nos & $\begin{array}{l}\text { Neurotic disorders, personality } \\
\text { disorders, and oth }\end{array}$ & $\mathrm{MH}$ & $\begin{array}{l}\text { Other Neurotic and } \\
\text { Nonpsychotic Mental } \\
\text { Disorders }\end{array}$ & $\begin{array}{l}\text { Personality Disorder } \\
\text { NOS }\end{array}$ & & & Personality \\
\hline 302 & Sexual Deviations And Disorders & $\begin{array}{l}\text { Neurotic disorders, personality } \\
\text { disorders, and oth }\end{array}$ & $\mathrm{MH}$ & $\begin{array}{l}\text { Other Neurotic and } \\
\text { Nonpsychotic Mental } \\
\text { Disorders }\end{array}$ & & & & Sexual \\
\hline 302.0 & Ego-Dystonic Homosexlty & $\begin{array}{l}\text { Neurotic disorders, personality } \\
\text { disorders, and oth }\end{array}$ & MH & $\begin{array}{l}\text { Other Neurotic and } \\
\text { Nonpsychotic Mental } \\
\text { Disorders }\end{array}$ & & & & Sexual \\
\hline 302.1 & Zoophilia & $\begin{array}{l}\text { Neurotic disorders, personality } \\
\text { disorders, and oth }\end{array}$ & $\mathrm{MH}$ & $\begin{array}{l}\text { Other Neurotic and } \\
\text { Nonpsychotic Mental } \\
\text { Disorders }\end{array}$ & & & & Sexual \\
\hline 302.2 & Pedophilia & $\begin{array}{l}\text { Neurotic disorders, personality } \\
\text { disorders, and oth }\end{array}$ & MH & $\begin{array}{l}\text { Other Neurotic and } \\
\text { Nonpsychotic Mental } \\
\text { Disorders }\end{array}$ & Pedophilia & & & Sexual \\
\hline
\end{tabular}




\begin{tabular}{|c|c|c|c|c|c|c|c|c|}
\hline \multicolumn{9}{|c|}{ APPENDIX } \\
\hline diagn1 & Description & Subclass & MH SA & DPW_Diagcat & DSM_IV_TR & $\begin{array}{c}\text { Abuse } \\
\text { Dependency }\end{array}$ & $\begin{array}{l}\text { Alcohol } \\
\text { Drug }\end{array}$ & Research_1A \\
\hline 302.20 & Invalid Code $-302.2=$ Pedophilia & $\begin{array}{l}\text { Neurotic disorders, personality } \\
\text { disorders, and oth }\end{array}$ & $\mathrm{MH}$ & $\begin{array}{l}\text { Other Neurotic and } \\
\text { Nonpsychotic Mental } \\
\text { Disorders }\end{array}$ & & & & Sexual \\
\hline 302.3 & Transvestism & $\begin{array}{l}\text { Neurotic disorders, personality } \\
\text { disorders, and oth }\end{array}$ & $\mathrm{MH}$ & $\begin{array}{l}\text { Other Neurotic and } \\
\text { Nonpsychotic Mental } \\
\text { Disorders }\end{array}$ & Transvestic Fetishism & & & Sexual \\
\hline 302.4 & Exhibitionism & $\begin{array}{l}\text { Neurotic disorders, personality } \\
\text { disorders, and oth }\end{array}$ & $\mathrm{MH}$ & $\begin{array}{l}\text { Other Neurotic and } \\
\text { Nonpsychotic Mental } \\
\text { Disorders }\end{array}$ & Exhibitionism & & & Sexual \\
\hline 302.50 & Trans-Sexualism Nos & $\begin{array}{l}\text { Neurotic disorders, personality } \\
\text { disorders, and oth }\end{array}$ & MH & $\begin{array}{l}\text { Other Neurotic and } \\
\text { Nonpsychotic Mental } \\
\text { Disorders }\end{array}$ & & & & Sexual \\
\hline 302.51 & Trans-Sexualism, Asexual & $\begin{array}{l}\text { Neurotic disorders, personality } \\
\text { disorders, and oth }\end{array}$ & $\mathrm{MH}$ & $\begin{array}{l}\text { Other Neurotic and } \\
\text { Nonpsychotic Mental } \\
\text { Disorders }\end{array}$ & & & & Sexual \\
\hline 302.52 & Trans-Sexual, Homosexual & $\begin{array}{l}\text { Neurotic disorders, personality } \\
\text { disorders, and oth }\end{array}$ & $\mathrm{MH}$ & $\begin{array}{l}\text { Other Neurotic and } \\
\text { Nonpsychotic Mental } \\
\text { Disorders }\end{array}$ & & & & Sexual \\
\hline 302.53 & Trans-Sex, Heterosexual & $\begin{array}{l}\text { Neurotic disorders, personality } \\
\text { disorders, and oth }\end{array}$ & MH & $\begin{array}{l}\text { Other Neurotic and } \\
\text { Nonpsychotic Mental } \\
\text { Disorders }\end{array}$ & & & & Sexual \\
\hline 302.6 & Psychosex Identity Dis & $\begin{array}{l}\text { Neurotic disorders, personality } \\
\text { disorders, and oth }\end{array}$ & $\mathrm{MH}$ & $\begin{array}{l}\text { Other Neurotic and } \\
\text { Nonpsychotic Mental } \\
\text { Disorders }\end{array}$ & $\begin{array}{l}\text { Gender Identity Disorder } \\
\text { [in Children/NOS] }\end{array}$ & & & Sexual \\
\hline 302.7 & Psychosexual Dysfunction & $\begin{array}{l}\text { Neurotic disorders, personality } \\
\text { disorders, and oth }\end{array}$ & $\mathrm{MH}$ & $\begin{array}{l}\text { Other Neurotic and } \\
\text { Nonpsychotic Mental } \\
\text { Disorders }\end{array}$ & & & & Sexual \\
\hline 302.70 & Psychosexual Dysfunc Nos & $\begin{array}{l}\text { Neurotic disorders, personality } \\
\text { disorders, and oth }\end{array}$ & $\mathrm{MH}$ & $\begin{array}{l}\text { Other Neurotic and } \\
\text { Nonpsychotic Mental } \\
\text { Disorders }\end{array}$ & Sexual Dysfunction NOS & & & Sexual \\
\hline 302.71 & Inhibited Sexual Desire & $\begin{array}{l}\text { Neurotic disorders, personality } \\
\text { disorders, and oth }\end{array}$ & $\mathrm{MH}$ & $\begin{array}{l}\text { Other Neurotic and } \\
\text { Nonpsychotic Mental } \\
\text { Disorders }\end{array}$ & $\begin{array}{l}\text { Hypoactive Sexual } \\
\text { Desire Disorder }\end{array}$ & & & Sexual \\
\hline 302.72 & Inhibited Sex Excitement & $\begin{array}{l}\text { Neurotic disorders, personality } \\
\text { disorders, and oth }\end{array}$ & $\mathrm{MH}$ & $\begin{array}{l}\text { Other Neurotic and } \\
\text { Nonpsychotic Mental } \\
\text { Disorders }\end{array}$ & $\begin{array}{l}\text { [Female Sexual } \\
\text { Arousal/Male Erectile] } \\
\text { Disorder }\end{array}$ & & & Sexual \\
\hline 302.73 & Inhibited Female Orgasm & $\begin{array}{l}\text { Neurotic disorders, personality } \\
\text { disorders, and oth }\end{array}$ & $\mathrm{MH}$ & $\begin{array}{l}\text { Other Neurotic and } \\
\text { Nonpsychotic Mental } \\
\text { Disorders }\end{array}$ & $\begin{array}{l}\text { Female Orgasmic } \\
\text { Disorder }\end{array}$ & & & Sexual \\
\hline 302.74 & Inhibited Male Orgasm & $\begin{array}{l}\text { Neurotic disorders, personality } \\
\text { disorders, and oth }\end{array}$ & MH & $\begin{array}{l}\text { Other Neurotic and } \\
\text { Nonpsychotic Mental } \\
\text { Disorders }\end{array}$ & Male Orgasmic Disorder & & & Sexual \\
\hline 302.75 & Premature Ejaculation & $\begin{array}{l}\text { Neurotic disorders, personality } \\
\text { disorders, and oth }\end{array}$ & MH & $\begin{array}{l}\text { Other Neurotic and } \\
\text { Nonpsychotic Mental } \\
\text { Disorders }\end{array}$ & Premature Ejaculation & & & Sexual \\
\hline 302.76 & Functional Dyspareunia & $\begin{array}{l}\text { Neurotic disorders, personality } \\
\text { disorders, and oth }\end{array}$ & $\mathrm{MH}$ & $\begin{array}{l}\text { Other Neurotic and } \\
\text { Nonpsychotic Mental } \\
\text { Disorders }\end{array}$ & $\begin{array}{l}\text { Dyspareunia (Not due to } \\
\text { a general medical } \\
\text { condition) }\end{array}$ & & & Sexual \\
\hline
\end{tabular}




\begin{tabular}{|c|c|c|c|c|c|c|c|c|}
\hline \multicolumn{9}{|c|}{ APPENDIX } \\
\hline diagn1 & Description & Subclass & MH SA & DPW_Diagcat & DSM_IV_TR & $\begin{array}{c}\text { Abuse } \\
\text { Dependency }\end{array}$ & $\begin{array}{l}\text { Alcohol } \\
\text { Drug }\end{array}$ & Research_1A \\
\hline 302.79 & Psychosexual Dysfunc Nec & $\begin{array}{l}\text { Neurotic disorders, personality } \\
\text { disorders, and oth }\end{array}$ & $\mathrm{MH}$ & $\begin{array}{l}\text { Other Neurotic and } \\
\text { Nonpsychotic Mental } \\
\text { Disorders }\end{array}$ & Sexual Aversion Disorder & & & Sexual \\
\hline 302.8 & $\begin{array}{l}\text { Other Specified Psychosexual } \\
\text { Disorders }\end{array}$ & $\begin{array}{l}\text { Neurotic disorders, personality } \\
\text { disorders, and oth }\end{array}$ & MH & $\begin{array}{l}\text { Other Neurotic and } \\
\text { Nonpsychotic Mental } \\
\text { Disorders }\end{array}$ & & & & Sexual \\
\hline 302.81 & Fetishism & $\begin{array}{l}\text { Neurotic disorders, personality } \\
\text { disorders, and oth }\end{array}$ & MH & $\begin{array}{l}\text { Other Neurotic and } \\
\text { Nonpsychotic Mental } \\
\text { Disorders }\end{array}$ & Fetishism & & & Sexual \\
\hline 302.82 & Voyeurism & $\begin{array}{l}\text { Neurotic disorders, personality } \\
\text { disorders, and oth }\end{array}$ & $\mathrm{MH}$ & $\begin{array}{l}\text { Other Neurotic and } \\
\text { Nonpsychotic Mental } \\
\text { Disorders } \\
\end{array}$ & Voyeurism & & & Sexual \\
\hline 302.83 & Sexual Masochism & $\begin{array}{l}\text { Neurotic disorders, personality } \\
\text { disorders, and oth }\end{array}$ & MH & $\begin{array}{l}\text { Other Neurotic and } \\
\text { Nonpsychotic Mental } \\
\text { Disorders }\end{array}$ & Sexual Masochism & & & Sexual \\
\hline 302.84 & Sexual Sadism & $\begin{array}{l}\text { Neurotic disorders, personality } \\
\text { disorders, and oth }\end{array}$ & $\mathrm{MH}$ & $\begin{array}{l}\text { Other Neurotic and } \\
\text { Nonpsychotic Mental } \\
\text { Disorders }\end{array}$ & Sexual Sadism & & & Sexual \\
\hline 302.85 & Gend Iden Dis,Adol Adult & $\begin{array}{l}\text { Neurotic disorders, personality } \\
\text { disorders, and oth }\end{array}$ & MH & $\begin{array}{l}\text { Other Neurotic and } \\
\text { Nonpsychotic Mental } \\
\text { Disorders }\end{array}$ & $\begin{array}{l}\text { Gender Identity Disorder } \\
\text { in Adolescents or Adults }\end{array}$ & & & Sexual \\
\hline 302.89 & Psychosexual Dis Nec & $\begin{array}{l}\text { Neurotic disorders, personality } \\
\text { disorders, and oth }\end{array}$ & MH & $\begin{array}{l}\text { Other Neurotic and } \\
\text { Nonpsychotic Mental } \\
\text { Disorders }\end{array}$ & Frotteurism & & & Sexual \\
\hline 302.9 & Psychosexual Dis Nos & $\begin{array}{l}\text { Neurotic disorders, personality } \\
\text { disorders, and oth }\end{array}$ & $\mathrm{MH}$ & $\begin{array}{l}\text { Other Neurotic and } \\
\text { Nonpsychotic Mental } \\
\text { Disorders }\end{array}$ & $\begin{array}{l}\text { [Paraphilia/Sexual } \\
\text { Disorder] NOS }\end{array}$ & & & Sexual \\
\hline 302.90 & $\begin{array}{l}\text { Invalid Code }-302.9= \\
\text { Psychosexual Dis Nos }\end{array}$ & $\begin{array}{l}\text { Neurotic disorders, personality } \\
\text { disorders, and oth }\end{array}$ & MH & $\begin{array}{l}\text { Other Neurotic and } \\
\text { Nonpsychotic Mental } \\
\text { Disorders }\end{array}$ & & & & Sexual \\
\hline 303 & Alcohol Dependence Syndrome & $\begin{array}{l}\text { Neurotic disorders, personality } \\
\text { disorders, and oth }\end{array}$ & SA & $\begin{array}{l}\text { Other Substance Related } \\
\text { Disorders }\end{array}$ & & & Alcohol & Alcohol Related Disorders \\
\hline 303.0 & Acute Alcoholic Intoxication & $\begin{array}{l}\text { Neurotic disorders, personality } \\
\text { disorders, and oth }\end{array}$ & SA & $\begin{array}{l}\text { Other Substance Related } \\
\text { Disorders }\end{array}$ & & & Alcohol & Alcohol Related Disorders \\
\hline 303.00 & Ac Alcohol Intox-Unspec & $\begin{array}{l}\text { Neurotic disorders, personality } \\
\text { disorders, and oth }\end{array}$ & SA & $\begin{array}{l}\text { Other Substance Related } \\
\text { Disorders }\end{array}$ & Alcohol Intoxication & & Alcohol & Alcohol Related Disorders \\
\hline 303.01 & Ac Alcohol Intox-Contin & $\begin{array}{l}\text { Neurotic disorders, personality } \\
\text { disorders, and oth }\end{array}$ & SA & $\begin{array}{l}\text { Other Substance Related } \\
\text { Disorders }\end{array}$ & & & Alcohol & Alcohol Related Disorders \\
\hline 303.02 & Ac Alcohol Intox-Episod & $\begin{array}{l}\text { Neurotic disorders, personality } \\
\text { disorders, and oth }\end{array}$ & SA & $\begin{array}{l}\text { Other Substance Related } \\
\text { Disorders }\end{array}$ & & & Alcohol & Alcohol Related Disorders \\
\hline 303.03 & Ac Alcohol Intox-Remiss & $\begin{array}{l}\text { Neurotic disorders, personality } \\
\text { disorders, and oth }\end{array}$ & SA & $\begin{array}{l}\text { Other Substance Related } \\
\text { Disorders }\end{array}$ & & & Alcohol & Alcohol Related Disorders \\
\hline 303.9 & $\begin{array}{l}\text { Other And Unspecified Alcohol } \\
\text { Dependence Chronic Alcoholism } \\
\text { Dipsomania }\end{array}$ & $\begin{array}{l}\text { Neurotic disorders, personality } \\
\text { disorders, and oth }\end{array}$ & SA & Alcohol Dependency & & Dependence & Alcohol & Alcohol Related Disorders \\
\hline 303.90 & Alcoh Dep Nec Nos-Unspec & $\begin{array}{l}\text { Neurotic disorders, personality } \\
\text { disorders, and oth }\end{array}$ & SA & Alcohol Dependency & Alcohol Dependence & Dependence & Alcohol & Alcohol Related Disorders \\
\hline 303.91 & Alcoh Dep Nec Nos-Contin & $\begin{array}{l}\text { Neurotic disorders, personality } \\
\text { disorders, and oth }\end{array}$ & SA & Alcohol Dependency & & Dependence & Alcohol & Alcohol Related Disorders \\
\hline
\end{tabular}


APPENDIX Crosswalk of Diagnostic Categories Between State Categories (DPW) to DSM-IV-TR to Research-IA Categories Used by Community Care Behavioral Health Organization (continued)

\begin{tabular}{|c|c|c|c|c|c|c|c|c|}
\hline diagn1 & Description & Subclass & MH SA & DPW_Diagcat & DSM_IV_TR & $\begin{array}{c}\text { Abuse } \\
\text { Dependency }\end{array}$ & $\begin{array}{l}\text { Alcohol } \\
\text { Drug }\end{array}$ & Research_1A \\
\hline 303.92 & Alcoh Dep Nec Nos-Episod & $\begin{array}{l}\text { Neurotic disorders, personality } \\
\text { disorders, and oth }\end{array}$ & SA & Alcohol Dependency & & Dependence & Alcohol & Alcohol Related Disorders \\
\hline 303.93 & Alcoh Dep Nec Nos-Remiss & $\begin{array}{l}\text { Neurotic disorders, personality } \\
\text { disorders, and oth }\end{array}$ & SA & Alcohol Dependency & & Dependence & Alcohol & Alcohol Related Disorders \\
\hline 304 & Drug Dependence & $\begin{array}{l}\text { Neurotic disorders, personality } \\
\text { disorders, and oth }\end{array}$ & SA & $\begin{array}{l}\text { Other Substance Related } \\
\text { Disorders }\end{array}$ & & Dependence & Drug & Drug Related Disorders \\
\hline 304.0 & Opioid Type Dependence & $\begin{array}{l}\text { Neurotic disorders, personality } \\
\text { disorders, and oth }\end{array}$ & SA & Opioid, Dependency & & Dependence & Drug & Opiod Related Disorders \\
\hline 304.00 & Opioid Dependence-Unspec & $\begin{array}{l}\text { Neurotic disorders, personality } \\
\text { disorders, and oth }\end{array}$ & SA & Opioid, Dependency & Opioid Dependence & Dependence & Drug & Opiod Related Disorders \\
\hline 304.01 & Opioid Dependence-Contin & $\begin{array}{l}\text { Neurotic disorders, personality } \\
\text { disorders, and oth }\end{array}$ & SA & Opioid, Dependency & & Dependence & Drug & Opiod Related Disorders \\
\hline 304.02 & Opioid Dependence-Episod & $\begin{array}{l}\text { Neurotic disorders, personality } \\
\text { disorders, and oth }\end{array}$ & SA & Opioid, Dependency & & Dependence & Drug & Opiod Related Disorders \\
\hline 304.03 & Opioid Dependence-Remiss & $\begin{array}{l}\text { Neurotic disorders, personality } \\
\text { disorders, and oth }\end{array}$ & SA & Opioid, Dependency & & Dependence & Drug & Opiod Related Disorders \\
\hline 304.1 & $\begin{array}{l}\text { Barbiturate And Similarly Acting } \\
\text { Sedative Or Hypnotic Dependence }\end{array}$ & $\begin{array}{l}\text { Neurotic disorders, personality } \\
\text { disorders, and oth }\end{array}$ & SA & $\begin{array}{l}\text { Sedat., Hypnotic, Anxiolytic, } \\
\text { Dependency }\end{array}$ & & Dependence & Drug & $\begin{array}{l}\text { Hypnotic Related } \\
\text { Disorders }\end{array}$ \\
\hline 304.10 & Barbiturat Depend-Unspec & $\begin{array}{l}\text { Neurotic disorders, personality } \\
\text { disorders, and oth }\end{array}$ & SA & $\begin{array}{l}\text { Sedat., Hypnotic, Anxiolytic, } \\
\text { Dependency }\end{array}$ & $\begin{array}{l}\text { Sedative, Hypnotic, or } \\
\text { Anxiolytic Dependence }\end{array}$ & Dependence & Drug & $\begin{array}{l}\text { Hypnotic Related } \\
\text { Disorders }\end{array}$ \\
\hline 304.11 & Barbiturat Depend-Contin & $\begin{array}{l}\text { Neurotic disorders, personality } \\
\text { disorders, and oth }\end{array}$ & SA & $\begin{array}{l}\text { Sedat., Hypnotic, Anxiolytic, } \\
\text { Dependency }\end{array}$ & & Dependence & Drug & $\begin{array}{l}\text { Hypnotic Related } \\
\text { Disorders }\end{array}$ \\
\hline 304.12 & Barbiturat Depend-Episod & $\begin{array}{l}\text { Neurotic disorders, personality } \\
\text { disorders, and oth }\end{array}$ & SA & $\begin{array}{l}\text { Sedat., Hypnotic, Anxiolytic, } \\
\text { Dependency }\end{array}$ & & Dependence & Drug & $\begin{array}{l}\text { Hypnotic Related } \\
\text { Disorders }\end{array}$ \\
\hline 304.13 & Barbiturat Depend-Remiss & $\begin{array}{l}\text { Neurotic disorders, personality } \\
\text { disorders, and oth }\end{array}$ & SA & $\begin{array}{l}\text { Sedat., Hypnotic, Anxiolytic, } \\
\text { Dependency }\end{array}$ & & Dependence & Drug & $\begin{array}{l}\text { Hypnotic Related } \\
\text { Disorders }\end{array}$ \\
\hline 304.2 & Cocaine Dependence & $\begin{array}{l}\text { Neurotic disorders, personality } \\
\text { disorders, and oth }\end{array}$ & SA & Cocaine/Crack, Dependency & & Dependence & Drug & $\begin{array}{l}\text { Cocaine Related } \\
\text { Disorders }\end{array}$ \\
\hline 304.20 & Cocaine Depend-Unspec & $\begin{array}{l}\text { Neurotic disorders, personality } \\
\text { disorders, and oth }\end{array}$ & SA & Cocaine/Crack, Dependency & Cocaine Dependence & Dependence & Drug & $\begin{array}{l}\text { Cocaine Related } \\
\text { Disorders }\end{array}$ \\
\hline 304.21 & Cocaine Depend-Contin & $\begin{array}{l}\text { Neurotic disorders, personality } \\
\text { disorders, and oth }\end{array}$ & SA & Cocaine/Crack, Dependency & & Dependence & Drug & $\begin{array}{l}\text { Cocaine Related } \\
\text { Disorders }\end{array}$ \\
\hline 304.22 & Cocaine Depend-Episodic & $\begin{array}{l}\text { Neurotic disorders, personality } \\
\text { disorders, and oth }\end{array}$ & SA & Cocaine/Crack, Dependency & & Dependence & Drug & $\begin{array}{l}\text { Cocaine Related } \\
\text { Disorders }\end{array}$ \\
\hline 304.23 & Cocaine Depend-Remiss & $\begin{array}{l}\text { Neurotic disorders, personality } \\
\text { disorders, and oth }\end{array}$ & SA & Cocaine/Crack, Dependency & & Dependence & Drug & $\begin{array}{l}\text { Cocaine Related } \\
\text { Disorders }\end{array}$ \\
\hline 304.3 & Cannabis Dependence & $\begin{array}{l}\text { Neurotic disorders, personality } \\
\text { disorders, and oth }\end{array}$ & SA & Cannabis, Dependency & & Dependence & Drug & $\begin{array}{l}\text { Cannabis Related } \\
\text { Disorders }\end{array}$ \\
\hline 304.30 & Cannabis Depend-Unspec & $\begin{array}{l}\text { Neurotic disorders, personality } \\
\text { disorders, and oth }\end{array}$ & SA & Cannabis, Dependency & Cannabis Dependence & Dependence & Drug & $\begin{array}{l}\text { Cannabis Related } \\
\text { Disorders }\end{array}$ \\
\hline 304.31 & Cannabis Depend-Contin & $\begin{array}{l}\text { Neurotic disorders, personality } \\
\text { disorders, and oth }\end{array}$ & SA & Cannabis, Dependency & & Dependence & Drug & $\begin{array}{l}\text { Cannabis Related } \\
\text { Disorders }\end{array}$ \\
\hline 304.32 & Cannabis Depend-Episodic & $\begin{array}{l}\text { Neurotic disorders, personality } \\
\text { disorders, and oth }\end{array}$ & SA & Cannabis, Dependency & & Dependence & Drug & $\begin{array}{l}\text { Cannabis Related } \\
\text { Disorders }\end{array}$ \\
\hline 304.33 & Cannabis Depend-Remiss & $\begin{array}{l}\text { Neurotic disorders, personality } \\
\text { disorders, and oth }\end{array}$ & SA & Cannabis, Dependency & & Dependence & Drug & $\begin{array}{l}\text { Cannabis Related } \\
\text { Disorders }\end{array}$ \\
\hline 304.4 & $\begin{array}{l}\text { Amphetamine And Other } \\
\text { Psychostimulant Dependence }\end{array}$ & $\begin{array}{l}\text { Neurotic disorders, personality } \\
\text { disorders, and oth }\end{array}$ & SA & Amphetamine, Dependency & & Dependence & Drug & $\begin{array}{l}\text { Amphetamine Related } \\
\text { Disorders }\end{array}$ \\
\hline 304.40 & Amphetamin Depend-Unspec & $\begin{array}{l}\text { Neurotic disorders, personality } \\
\text { disorders, and oth }\end{array}$ & SA & Amphetamine, Dependency & $\begin{array}{l}\text { Amphetamine } \\
\text { Dependence }\end{array}$ & Dependence & Drug & $\begin{array}{l}\text { Amphetamine Related } \\
\text { Disorders }\end{array}$ \\
\hline
\end{tabular}


APPENDIX Crosswalk of Diagnostic Categories Between State Categories (DPW) to DSM-IV-TR to Research-IA Categories Used by Community Care Behavioral Health Organization (continued)

\begin{tabular}{|c|c|c|c|c|c|c|c|c|}
\hline diagn1 & Description & Subclass & MH SA & DPW_Diagcat & DSM_IV_TR & $\begin{array}{c}\text { Abuse } \\
\text { Dependency }\end{array}$ & $\begin{array}{l}\text { Alcohol } \\
\text { Drug }\end{array}$ & Research_1A \\
\hline 304.41 & Amphetamin Depend-Contin & $\begin{array}{l}\text { Neurotic disorders, personality } \\
\text { disorders, and oth }\end{array}$ & SA & Amphetamine, Dependency & & Dependence & Drug & $\begin{array}{l}\text { Amphetamine Related } \\
\text { Disorders }\end{array}$ \\
\hline 304.42 & Amphetamin Depend-Episod & $\begin{array}{l}\text { Neurotic disorders, personality } \\
\text { disorders, and oth }\end{array}$ & SA & Amphetamine, Dependency & & Dependence & Drug & $\begin{array}{l}\text { Amphetamine Related } \\
\text { Disorders }\end{array}$ \\
\hline 304.43 & Amphetamin Depend-Remiss & $\begin{array}{l}\text { Neurotic disorders, personality } \\
\text { disorders, and oth }\end{array}$ & SA & Amphetamine, Dependency & & Dependence & Drug & $\begin{array}{l}\text { Amphetamine Related } \\
\text { Disorders }\end{array}$ \\
\hline 304.5 & Hallucinogen Dependence & $\begin{array}{l}\text { Neurotic disorders, personality } \\
\text { disorders, and oth }\end{array}$ & SA & Hallucinogen, Dependency & & Dependence & Drug & $\begin{array}{l}\text { Hallucinogen Related } \\
\text { Disorders }\end{array}$ \\
\hline 304.50 & Hallucinogen Dep-Unspec & $\begin{array}{l}\text { Neurotic disorders, personality } \\
\text { disorders, and oth }\end{array}$ & SA & Hallucinogen, Dependency & $\begin{array}{l}\text { Hallucinogen } \\
\text { Dependence }\end{array}$ & Dependence & Drug & $\begin{array}{l}\text { Hallucinogen Related } \\
\text { Disorders }\end{array}$ \\
\hline 304.51 & Hallucinogen Dep-Contin & $\begin{array}{l}\text { Neurotic disorders, personality } \\
\text { disorders, and oth }\end{array}$ & SA & Hallucinogen, Dependency & & Dependence & Drug & $\begin{array}{l}\text { Hallucinogen Related } \\
\text { Disorders }\end{array}$ \\
\hline 304.52 & Hallucinogen Dep-Episod & $\begin{array}{l}\text { Neurotic disorders, personality } \\
\text { disorders, and oth }\end{array}$ & SA & Hallucinogen, Dependency & & Dependence & Drug & $\begin{array}{l}\text { Hallucinogen Related } \\
\text { Disorders }\end{array}$ \\
\hline 304.53 & Hallucinogen Dep-Remiss & $\begin{array}{l}\text { Neurotic disorders, personality } \\
\text { disorders, and oth }\end{array}$ & SA & Hallucinogen, Dependency & & Dependence & Drug & $\begin{array}{l}\text { Hallucinogen Related } \\
\text { Disorders }\end{array}$ \\
\hline 304.6 & Other Specified Drug Dependence & $\begin{array}{l}\text { Neurotic disorders, personality } \\
\text { disorders, and oth }\end{array}$ & SA & Inhalant, Dependency & & Dependence & Drug & $\begin{array}{l}\text { Inhalant Related } \\
\text { Disorders }\end{array}$ \\
\hline 304.60 & Drug Depend Nec-Unspec & $\begin{array}{l}\text { Neurotic disorders, personality } \\
\text { disorders, and oth }\end{array}$ & SA & Inhalant, Dependency & $\begin{array}{l}\text { [Inhalant/Phencyclidine] } \\
\text { Dependence }\end{array}$ & Dependence & Drug & $\begin{array}{l}\text { Inhalant Related } \\
\text { Disorders }\end{array}$ \\
\hline 304.61 & Drug Depend Nec-Contin & $\begin{array}{l}\text { Neurotic disorders, personality } \\
\text { disorders, and oth }\end{array}$ & SA & Inhalant, Dependency & & Dependence & Drug & $\begin{array}{l}\text { Inhalant Related } \\
\text { Disorders }\end{array}$ \\
\hline 304.62 & Drug Depend Nec-Episodic & $\begin{array}{l}\text { Neurotic disorders, personality } \\
\text { disorders, and oth }\end{array}$ & SA & Inhalant, Dependency & & Dependence & Drug & $\begin{array}{l}\text { Inhalant Related } \\
\text { Disorders }\end{array}$ \\
\hline 304.63 & Drug Depend Nec-In Rem & $\begin{array}{l}\text { Neurotic disorders, personality } \\
\text { disorders, and oth }\end{array}$ & SA & Inhalant, Dependency & & Dependence & Drug & $\begin{array}{l}\text { Inhalant Related } \\
\text { Disorders }\end{array}$ \\
\hline 304.7 & $\begin{array}{l}\text { Combinations Of Opioid Type Drug } \\
\text { With Any Other }\end{array}$ & $\begin{array}{l}\text { Neurotic disorders, personality } \\
\text { disorders, and oth }\end{array}$ & SA & $\begin{array}{l}\text { Other Substance Related } \\
\text { Disorders }\end{array}$ & & Dependence & Drug & Opiod Related Disorders \\
\hline 304.70 & Opioid Other Dep-Unspec & $\begin{array}{l}\text { Neurotic disorders, personality } \\
\text { disorders, and oth }\end{array}$ & SA & $\begin{array}{l}\text { Other Substance Related } \\
\text { Disorders }\end{array}$ & & Dependence & Drug & Opiod Related Disorders \\
\hline 304.71 & Opioid Other Dep-Contin & $\begin{array}{l}\text { Neurotic disorders, personality } \\
\text { disorders, and oth }\end{array}$ & SA & $\begin{array}{l}\text { Other Substance Related } \\
\text { Disorders }\end{array}$ & & Dependence & Drug & Opiod Related Disorders \\
\hline 304.72 & Opioid Other Dep-Episod & $\begin{array}{l}\text { Neurotic disorders, personality } \\
\text { disorders, and oth }\end{array}$ & SA & $\begin{array}{l}\text { Other Substance Related } \\
\text { Disorders }\end{array}$ & & Dependence & Drug & Opiod Related Disorders \\
\hline 304.73 & Opioid Other Dep-Remiss & $\begin{array}{l}\text { Neurotic disorders, personality } \\
\text { disorders, and oth }\end{array}$ & SA & $\begin{array}{l}\text { Other Substance Related } \\
\text { Disorders }\end{array}$ & & Dependence & Drug & Opiod Related Disorders \\
\hline 304.8 & $\begin{array}{l}\text { Combinations Of Drug Dependence } \\
\text { Excluding Opioid Type Drug }\end{array}$ & $\begin{array}{l}\text { Neurotic disorders, personality } \\
\text { disorders, and oth }\end{array}$ & SA & $\begin{array}{l}\text { Other Substance Related } \\
\text { Disorders }\end{array}$ & & Dependence & Drug & Drug Related Disorders \\
\hline 304.80 & Comb Drug Dep Nec-Unspec & $\begin{array}{l}\text { Neurotic disorders, personality } \\
\text { disorders, and oth }\end{array}$ & SA & $\begin{array}{l}\text { Other Substance Related } \\
\text { Disorders }\end{array}$ & $\begin{array}{l}\text { Polysubstance } \\
\text { Dependence }\end{array}$ & Dependence & Drug & Drug Related Disorders \\
\hline 304.81 & Comb Drug Dep Nec-Contin & $\begin{array}{l}\text { Neurotic disorders, personality } \\
\text { disorders, and oth }\end{array}$ & SA & $\begin{array}{l}\text { Other Substance Related } \\
\text { Disorders }\end{array}$ & & Dependence & Drug & Drug Related Disorders \\
\hline 304.82 & Comb Drug Dep Nec-Episod & $\begin{array}{l}\text { Neurotic disorders, personality } \\
\text { disorders, and oth }\end{array}$ & SA & $\begin{array}{l}\text { Other Substance Related } \\
\text { Disorders }\end{array}$ & & Dependence & Drug & Drug Related Disorders \\
\hline 304.83 & Comb Drug Dep Nec-Remiss & $\begin{array}{l}\text { Neurotic disorders, personality } \\
\text { disorders, and oth }\end{array}$ & SA & $\begin{array}{l}\text { Other Substance Related } \\
\text { Disorders }\end{array}$ & & Dependence & Drug & Drug Related Disorders \\
\hline 304.9 & Drug Addiction Nos & $\begin{array}{l}\text { Neurotic disorders, personality } \\
\text { disorders, and oth }\end{array}$ & SA & $\begin{array}{l}\text { PCP or Polysubstance, } \\
\text { Dependency }\end{array}$ & & Dependence & Drug & Drug Related Disorders \\
\hline
\end{tabular}


APPENDIX Crosswalk of Diagnostic Categories Between State Categories (DPW) to DSM-IV-TR to Research-IA Categories Used by Community Care Behavioral Health Organization (continued)

\begin{tabular}{|c|c|c|c|c|c|c|c|c|}
\hline diagn1 & Description & Subclass & MH SA & DPW_Diagcat & DSM_IV_TR & $\begin{array}{c}\text { Abuse } \\
\text { Dependency }\end{array}$ & $\begin{array}{c}\text { Alcohol } \\
\text { Drug }\end{array}$ & Research_1A \\
\hline 304.90 & Drug Depend Nos-Unspec & $\begin{array}{l}\text { Neurotic disorders, personality } \\
\text { disorders, and oth }\end{array}$ & SA & $\begin{array}{l}\text { PCP or Polysubstance, } \\
\text { Dependency }\end{array}$ & $\begin{array}{l}\text { Other (or Unknown) } \\
\text { Substance Dependence }\end{array}$ & Dependence & Drug & Drug Related Disorders \\
\hline 304.91 & Drug Depend Nos-Contin & $\begin{array}{l}\text { Neurotic disorders, personality } \\
\text { disorders, and oth }\end{array}$ & SA & $\begin{array}{l}\text { PCP or Polysubstance, } \\
\text { Dependency }\end{array}$ & & Dependence & Drug & Drug Related Disorders \\
\hline 304.92 & Drug Depend Nos-Episodic & $\begin{array}{l}\text { Neurotic disorders, personality } \\
\text { disorders, and oth }\end{array}$ & SA & $\begin{array}{l}\text { PCP or Polysubstance, } \\
\text { Dependency }\end{array}$ & & Dependence & Drug & Drug Related Disorders \\
\hline 304.93 & Drug Depend Nos-Remiss & $\begin{array}{l}\text { Neurotic disorders, personality } \\
\text { disorders, and oth }\end{array}$ & SA & $\begin{array}{l}\text { PCP or Polysubstance, } \\
\text { Dependency }\end{array}$ & & Dependence & Drug & Drug Related Disorders \\
\hline 305 & Nondependent Abuse Of Drugs & $\begin{array}{l}\text { Neurotic disorders, personality } \\
\text { disorders, and oth }\end{array}$ & SA & $\begin{array}{l}\text { Other Substance Related } \\
\text { Disorders }\end{array}$ & & Abuse & Drug & Drug Related Disorders \\
\hline 305.0 & Alcohol Abuse & $\begin{array}{l}\text { Neurotic disorders, personality } \\
\text { disorders, and oth }\end{array}$ & SA & Alcohol Abuse & & Abuse & Alcohol & Alcohol Related Disorders \\
\hline 305.00 & Alcohol Abuse-Unspec & $\begin{array}{l}\text { Neurotic disorders, personality } \\
\text { disorders, and oth }\end{array}$ & SA & Alcohol Abuse & Alcohol Abuse & Abuse & Alcohol & Alcohol Related Disorders \\
\hline 305.01 & Alcohol Abuse-Continuous & $\begin{array}{l}\text { Neurotic disorders, personality } \\
\text { disorders, and oth }\end{array}$ & SA & Alcohol Abuse & & Abuse & Alcohol & Alcohol Related Disorders \\
\hline 305.02 & Alcohol Abuse-Episodic & $\begin{array}{l}\text { Neurotic disorders, personality } \\
\text { disorders, and oth }\end{array}$ & SA & Alcohol Abuse & & Abuse & Alcohol & Alcohol Related Disorders \\
\hline 305.03 & Alcohol Abuse-In Remiss & $\begin{array}{l}\text { Neurotic disorders, personality } \\
\text { disorders, and oth }\end{array}$ & SA & Alcohol Abuse & & Abuse & Alcohol & Alcohol Related Disorders \\
\hline 305.1 & Tobacco Use Disorder & $\begin{array}{l}\text { Neurotic disorders, personality } \\
\text { disorders, and oth }\end{array}$ & SA & $\begin{array}{l}\text { Other Substance Related } \\
\text { Disorders }\end{array}$ & Nicotine Dependence & & & $\begin{array}{l}\text { Tobacco Related } \\
\text { Disorders }\end{array}$ \\
\hline 305.10 & $\begin{array}{l}\text { Invalid Code - } 305.1=\text { Tobacco Use } \\
\text { Disorder }\end{array}$ & $\begin{array}{l}\text { Neurotic disorders, personality } \\
\text { disorders, and oth }\end{array}$ & SA & $\begin{array}{l}\text { Other Substance Related } \\
\text { Disorders }\end{array}$ & & & & $\begin{array}{l}\text { Tobacco Related } \\
\text { Disorders }\end{array}$ \\
\hline 305.12 & $\begin{array}{l}\text { Invalid Code - } 305.1 \text { = Tobacco Use } \\
\text { Disorder }\end{array}$ & $\begin{array}{l}\text { Neurotic disorders, personality } \\
\text { disorders, and oth }\end{array}$ & SA & $\begin{array}{l}\text { Other Substance Related } \\
\text { Disorders }\end{array}$ & & & & $\begin{array}{l}\text { Tobacco Related } \\
\text { Disorders }\end{array}$ \\
\hline 305.13 & $\begin{array}{l}\text { Invalid Code - } 305.1=\text { Tobacco Use } \\
\text { Disorder }\end{array}$ & Mental Disorders & SA & $\begin{array}{l}\text { Other Substance Related } \\
\text { Disorders }\end{array}$ & & & & $\begin{array}{l}\text { Tobacco Related } \\
\text { Disorders }\end{array}$ \\
\hline 305.2 & Cannabis Abuse & $\begin{array}{l}\text { Neurotic disorders, personality } \\
\text { disorders, and oth }\end{array}$ & SA & Cannabis, Abuse & & Abuse & Drug & $\begin{array}{l}\text { Cannabis Related } \\
\text { Disorders }\end{array}$ \\
\hline 305.20 & Cannabis Abuse-Unspec & $\begin{array}{l}\text { Neurotic disorders, personality } \\
\text { disorders, and oth }\end{array}$ & SA & Cannabis, Abuse & Cannabis Abuse & Abuse & Drug & $\begin{array}{l}\text { Cannabis Related } \\
\text { Disorders }\end{array}$ \\
\hline 305.21 & Cannabis Abuse-Contin & $\begin{array}{l}\text { Neurotic disorders, personality } \\
\text { disorders, and oth }\end{array}$ & SA & Cannabis, Abuse & & Abuse & Drug & $\begin{array}{l}\text { Cannabis Related } \\
\text { Disorders }\end{array}$ \\
\hline 305.22 & Cannabis Abuse-Episodic & $\begin{array}{l}\text { Neurotic disorders, personality } \\
\text { disorders, and oth }\end{array}$ & SA & Cannabis, Abuse & & Abuse & Drug & $\begin{array}{l}\text { Cannabis Related } \\
\text { Disorders }\end{array}$ \\
\hline 305.23 & Cannabis Abuse-In Remiss & $\begin{array}{l}\text { Neurotic disorders, personality } \\
\text { disorders, and oth }\end{array}$ & SA & Cannabis, Abuse & & Abuse & Drug & $\begin{array}{l}\text { Cannabis Related } \\
\text { Disorders }\end{array}$ \\
\hline 305.3 & Hallucinogen Abuse & $\begin{array}{l}\text { Neurotic disorders, personality } \\
\text { disorders, and oth }\end{array}$ & SA & Hallucinogen, Abuse & & Abuse & Drug & $\begin{array}{l}\text { Hullucinogen Related } \\
\text { Disorders }\end{array}$ \\
\hline 305.30 & Hallucinog Abuse-Unspec & $\begin{array}{l}\text { Neurotic disorders, personality } \\
\text { disorders, and oth }\end{array}$ & SA & Hallucinogen, Abuse & Hallucinogen Abuse & Abuse & Drug & $\begin{array}{l}\text { Hullucinogen Related } \\
\text { Disorders }\end{array}$ \\
\hline 305.31 & Hallucinog Abuse-Contin & $\begin{array}{l}\text { Neurotic disorders, personality } \\
\text { disorders, and oth }\end{array}$ & SA & Hallucinogen, Abuse & & Abuse & Drug & $\begin{array}{l}\text { Hullucinogen Related } \\
\text { Disorders }\end{array}$ \\
\hline 305.32 & Hallucinog Abuse-Episod & $\begin{array}{l}\text { Neurotic disorders, personality } \\
\text { disorders, and oth }\end{array}$ & SA & Hallucinogen, Abuse & & Abuse & Drug & $\begin{array}{l}\text { Hullucinogen Related } \\
\text { Disorders }\end{array}$ \\
\hline 305.33 & Hallucinog Abuse-Remiss & $\begin{array}{l}\text { Neurotic disorders, personality } \\
\text { disorders, and oth }\end{array}$ & SA & Hallucinogen, Abuse & & Abuse & Drug & $\begin{array}{l}\text { Hullucinogen Related } \\
\text { Disorders }\end{array}$ \\
\hline 305.4 & $\begin{array}{l}\text { Barbiturate And Similarly Acting } \\
\text { Sedative Or Hypnotic Abuse }\end{array}$ & $\begin{array}{l}\text { Neurotic disorders, personality } \\
\text { disorders, and oth }\end{array}$ & SA & $\begin{array}{l}\text { Sedat., Hypnotic, Anxiolytic, } \\
\text { Abuse }\end{array}$ & & Abuse & Drug & Hypnotic Related Disorde \\
\hline
\end{tabular}


APPENDIX Crosswalk of Diagnostic Categories Between State Categories (DPW) to DSM-IV-TR to Research-IA Categories Used by Community Care Behavioral Health Organization (continued)

\begin{tabular}{|c|c|c|c|c|c|c|c|c|}
\hline diagn1 & Description & Subclass & MH SA & DPW_Diagcat & DSM_IV_TR & $\begin{array}{c}\text { Abuse } \\
\text { Dependency }\end{array}$ & $\begin{array}{l}\text { Alcohol } \\
\text { Drug }\end{array}$ & Research_1A \\
\hline 305.40 & Barbiturate Abuse-Unspec & $\begin{array}{l}\text { Neurotic disorders, personality } \\
\text { disorders, and oth }\end{array}$ & SA & $\begin{array}{l}\text { Sedat., Hypnotic, Anxiolytic, } \\
\text { Abuse }\end{array}$ & $\begin{array}{l}\text { Sedative, Hypnotic, or } \\
\text { Anxiolytic Abuse }\end{array}$ & Abuse & Drug & Hypnotic Related Disorder \\
\hline 305.41 & Barbiturate Abuse-Contin & $\begin{array}{l}\text { Neurotic disorders, personality } \\
\text { disorders, and oth }\end{array}$ & SA & $\begin{array}{l}\text { Sedat., Hypnotic, Anxiolytic, } \\
\text { Abuse }\end{array}$ & & Abuse & Drug & Hypnotic Related Disorder \\
\hline 305.42 & Barbiturate Abuse-Episod & $\begin{array}{l}\text { Neurotic disorders, personality } \\
\text { disorders, and oth }\end{array}$ & SA & $\begin{array}{l}\text { Sedat., Hypnotic, Anxiolytic, } \\
\text { Abuse }\end{array}$ & & Abuse & Drug & Hypnotic Related Disorder \\
\hline 305.43 & Barbiturate Abuse-Remiss & $\begin{array}{l}\text { Neurotic disorders, personality } \\
\text { disorders, and oth }\end{array}$ & SA & $\begin{array}{l}\text { Sedat., Hypnotic, Anxiolytic, } \\
\text { Abuse }\end{array}$ & & Abuse & Drug & Hypnotic Related Disorder \\
\hline 305.5 & Opioid Abuse & $\begin{array}{l}\text { Neurotic disorders, personality } \\
\text { disorders, and oth }\end{array}$ & SA & Opioid, Abuse & & Abuse & Drug & Opiod Related Disorders \\
\hline 305.50 & Opioid Abuse-Unspec & $\begin{array}{l}\text { Neurotic disorders, personality } \\
\text { disorders, and oth }\end{array}$ & SA & Opioid, Abuse & Opioid Abuse & Abuse & Drug & Opiod Related Disorders \\
\hline 305.51 & Opioid Abuse-Continuous & $\begin{array}{l}\text { Neurotic disorders, personality } \\
\text { disorders, and oth }\end{array}$ & SA & Opioid, Abuse & & Abuse & Drug & Opiod Related Disorders \\
\hline 305.52 & Opioid Abuse-Episodic & $\begin{array}{l}\text { Neurotic disorders, personality } \\
\text { disorders, and oth }\end{array}$ & SA & Opioid, Abuse & & Abuse & Drug & Opiod Related Disorders \\
\hline 305.53 & Opioid Abuse-In Remiss & $\begin{array}{l}\text { Neurotic disorders, personality } \\
\text { disorders, and oth }\end{array}$ & SA & Opioid, Abuse & & Abuse & Drug & Opiod Related Disorders \\
\hline 305.6 & Cocaine Abuse & $\begin{array}{l}\text { Neurotic disorders, personality } \\
\text { disorders, and oth }\end{array}$ & SA & Cocaine/Crack, Abuse & & Abuse & Drug & $\begin{array}{l}\text { Cocaine Related } \\
\text { Disorders }\end{array}$ \\
\hline 305.60 & Cocaine Abuse-Unspec & $\begin{array}{l}\text { Neurotic disorders, personality } \\
\text { disorders, and oth }\end{array}$ & SA & Cocaine/Crack, Abuse & Cocaine Abuse & Abuse & Drug & $\begin{array}{l}\text { Cocaine Related } \\
\text { Disorders }\end{array}$ \\
\hline 305.61 & Cocaine Abuse-Continuous & $\begin{array}{l}\text { Neurotic disorders, personality } \\
\text { disorders, and oth }\end{array}$ & SA & Cocaine/Crack, Abuse & & Abuse & Drug & $\begin{array}{l}\text { Cocaine Related } \\
\text { Disorders }\end{array}$ \\
\hline 305.62 & Cocaine Abuse-Episodic & $\begin{array}{l}\text { Neurotic disorders, personality } \\
\text { disorders, and oth }\end{array}$ & SA & Cocaine/Crack, Abuse & & Abuse & Drug & $\begin{array}{l}\text { Cocaine Related } \\
\text { Disorders }\end{array}$ \\
\hline 305.63 & Cocaine Abuse-In Remiss & $\begin{array}{l}\text { Neurotic disorders, personality } \\
\text { disorders, and oth }\end{array}$ & SA & Cocaine/Crack, Abuse & & Abuse & Drug & $\begin{array}{l}\text { Cocaine Related } \\
\text { Disorders }\end{array}$ \\
\hline 305.7 & $\begin{array}{l}\text { Invalid Code }-305.70= \\
\text { Amphetamine Abuse-Unspec }\end{array}$ & $\begin{array}{l}\text { Neurotic disorders, personality } \\
\text { disorders, and oth }\end{array}$ & SA & Amphetamine, Abuse & & Abuse & Drug & $\begin{array}{l}\text { Amphetamine Related } \\
\text { Disorders }\end{array}$ \\
\hline 305.70 & Amphetamine Abuse-Unspec & $\begin{array}{l}\text { Neurotic disorders, personality } \\
\text { disorders, and oth }\end{array}$ & SA & Amphetamine, Abuse & Amphetamine Abuse & Abuse & Drug & $\begin{array}{l}\text { Amphetamine Related } \\
\text { Disorders }\end{array}$ \\
\hline 305.71 & Amphetamine Abuse-Contin & $\begin{array}{l}\text { Neurotic disorders, personality } \\
\text { disorders, and oth }\end{array}$ & SA & Amphetamine, Abuse & & Abuse & Drug & $\begin{array}{l}\text { Amphetamine Related } \\
\text { Disorders }\end{array}$ \\
\hline 305.72 & Amphetamine Abuse-Episod & $\begin{array}{l}\text { Neurotic disorders, personality } \\
\text { disorders, and oth }\end{array}$ & SA & Amphetamine, Abuse & & Abuse & Drug & $\begin{array}{l}\text { Amphetamine Related } \\
\text { Disorders }\end{array}$ \\
\hline 305.73 & Amphetamine Abuse-Remiss & $\begin{array}{l}\text { Neurotic disorders, personality } \\
\text { disorders, and oth }\end{array}$ & SA & Amphetamine, Abuse & & Abuse & Drug & $\begin{array}{l}\text { Amphetamine Related } \\
\text { Disorders }\end{array}$ \\
\hline 305.8 & Antidepressant Type Abuse & $\begin{array}{l}\text { Neurotic disorders, personality } \\
\text { disorders, and oth }\end{array}$ & SA & $\begin{array}{l}\text { Other Substance Related } \\
\text { Disorders }\end{array}$ & & Abuse & Drug & Antidepressant Abuse \\
\hline 305.80 & Antidepress Abuse-Unspec & $\begin{array}{l}\text { Neurotic disorders, personality } \\
\text { disorders, and oth }\end{array}$ & SA & $\begin{array}{l}\text { Other Substance Related } \\
\text { Disorders }\end{array}$ & & Abuse & Drug & Drug Related Disorders \\
\hline 305.81 & Antidepress Abuse-Contin & $\begin{array}{l}\text { Neurotic disorders, personality } \\
\text { disorders, and oth }\end{array}$ & SA & $\begin{array}{l}\text { Other Substance Related } \\
\text { Disorders }\end{array}$ & & Abuse & Drug & Drug Related Disorders \\
\hline 305.82 & Antidepress Abuse-Episod & $\begin{array}{l}\text { Neurotic disorders, personality } \\
\text { disorders, and oth }\end{array}$ & SA & $\begin{array}{l}\text { Other Substance Related } \\
\text { Disorders }\end{array}$ & & Abuse & Drug & Drug Related Disorders \\
\hline 305.83 & Antidepress Abuse-Remiss & $\begin{array}{l}\text { Neurotic disorders, personality } \\
\text { disorders, and oth }\end{array}$ & SA & $\begin{array}{l}\text { Other Substance Related } \\
\text { Disorders }\end{array}$ & & Abuse & Drug & Drug Related Disorders \\
\hline 305.9 & $\begin{array}{l}\text { Other, Mixed, Or Unspecified Drug } \\
\text { Abuse }\end{array}$ & $\begin{array}{l}\text { Neurotic disorders, personality } \\
\text { disorders, and oth }\end{array}$ & SA & Inhalant, Abuse & & Abuse & Drug & Drug Related Disorders \\
\hline
\end{tabular}




\begin{tabular}{|c|c|c|c|c|c|c|c|c|}
\hline \multicolumn{9}{|c|}{ APPENDIX } \\
\hline diagn1 & Description & Subclass & MH SA & DPW_Diagcat & DSM_IV_TR & \begin{tabular}{|c|} 
Abuse \\
Dependency
\end{tabular} & $\begin{array}{l}\text { Alcohol } \\
\text { Drug }\end{array}$ & Research_1A \\
\hline 305.90 & Drug Abuse Nec-Unspec & $\begin{array}{l}\text { Neurotic disorders, personality } \\
\text { disorders, and oth }\end{array}$ & SA & Inhalant, Abuse & $\begin{array}{l}\text { [Caffeine } \\
\text { Intoxication/Inhalant } \\
\text { Abuse/Other (or } \\
\text { Unknown) Substance } \\
\text { Abuse/Phencyclidine } \\
\text { Abuse] }\end{array}$ & Abuse & Drug & Drug Related Disorders \\
\hline 305.91 & Drug Abuse Nec-Contin & $\begin{array}{l}\text { Neurotic disorders, personality } \\
\text { disorders, and oth }\end{array}$ & SA & Inhalant, Abuse & & Abuse & Drug & Drug Related Disorders \\
\hline 305.92 & Drug Abuse Nec-Episodic & $\begin{array}{l}\text { Neurotic disorders, personality } \\
\text { disorders, and oth }\end{array}$ & SA & Inhalant, Abuse & & Abuse & Drug & Drug Related Disorders \\
\hline 305.93 & Drug Abuse Nec-In Remiss & $\begin{array}{l}\text { Neurotic disorders, personality } \\
\text { disorders, and oth }\end{array}$ & SA & Inhalant, Abuse & & Abuse & Drug & Drug Related Disorders \\
\hline 306 & $\begin{array}{l}\text { Physiological Malfunction Arising } \\
\text { From Mental Factors }\end{array}$ & $\begin{array}{l}\text { Neurotic disorders, personality } \\
\text { disorders, and oth }\end{array}$ & $\mathrm{MH}$ & $\begin{array}{l}\text { Other Neurotic and } \\
\text { Nonpsychotic Mental } \\
\text { Disorders }\end{array}$ & & & & Somatoform \\
\hline 306.0 & Psychogen Musculskel Dis & $\begin{array}{l}\text { Neurotic disorders, personality } \\
\text { disorders, and oth }\end{array}$ & $\mathrm{MH}$ & $\begin{array}{l}\text { Other Neurotic and } \\
\text { Nonpsychotic Mental } \\
\text { Disorders }\end{array}$ & & & & Somatoform \\
\hline 306.1 & Psychogenic Respir Dis & $\begin{array}{l}\text { Neurotic disorders, personality } \\
\text { disorders, and oth }\end{array}$ & $\mathrm{MH}$ & $\begin{array}{l}\text { Other Neurotic and } \\
\text { Nonpsychotic Mental } \\
\text { Disorders }\end{array}$ & & & & Somatoform \\
\hline 306.2 & Psychogen Cardiovasc Dis & $\begin{array}{l}\text { Neurotic disorders, personality } \\
\text { disorders, and oth }\end{array}$ & $\mathrm{MH}$ & $\begin{array}{l}\text { Other Neurotic and } \\
\text { Nonpsychotic Mental } \\
\text { Disorders }\end{array}$ & & & & Somatoform \\
\hline 306.3 & Psychogenic Skin Disease & $\begin{array}{l}\text { Neurotic disorders, personality } \\
\text { disorders, and oth }\end{array}$ & $\mathrm{MH}$ & $\begin{array}{l}\text { Other Neurotic and } \\
\text { Nonpsychotic Mental } \\
\text { Disorders }\end{array}$ & & & & Somatoform \\
\hline 306.4 & Psychogenic Gi Disease & $\begin{array}{l}\text { Neurotic disorders, personality } \\
\text { disorders, and oth }\end{array}$ & $\mathrm{MH}$ & $\begin{array}{l}\text { Other Neurotic and } \\
\text { Nonpsychotic Mental } \\
\text { Disorders }\end{array}$ & & & & Somatoform \\
\hline 306.50 & Psychogenic Gu Dis Nos & $\begin{array}{l}\text { Neurotic disorders, personality } \\
\text { disorders, and oth }\end{array}$ & $\mathrm{MH}$ & $\begin{array}{l}\text { Other Neurotic and } \\
\text { Nonpsychotic Mental } \\
\text { Disorders }\end{array}$ & & & & Somatoform \\
\hline 306.51 & Psychogenic Vaginismus & $\begin{array}{l}\text { Neurotic disorders, personality } \\
\text { disorders, and oth }\end{array}$ & $\mathrm{MH}$ & $\begin{array}{l}\text { Other Neurotic and } \\
\text { Nonpsychotic Mental } \\
\text { Disorders }\end{array}$ & $\begin{array}{l}\text { Vaginismus (Not Due to a } \\
\text { General Medical } \\
\text { Condition) }\end{array}$ & & & Somatoform \\
\hline 306.52 & Psychogenic Dysmenorrhea & $\begin{array}{l}\text { Neurotic disorders, personality } \\
\text { disorders, and oth }\end{array}$ & $\mathrm{MH}$ & $\begin{array}{l}\text { Other Neurotic and } \\
\text { Nonpsychotic Mental } \\
\text { Disorders }\end{array}$ & & & & Somatoform \\
\hline 306.53 & Psychogenic Dysuria & $\begin{array}{l}\text { Neurotic disorders, personality } \\
\text { disorders, and oth }\end{array}$ & $\mathrm{MH}$ & $\begin{array}{l}\text { Other Neurotic and } \\
\text { Nonpsychotic Mental } \\
\text { Disorders }\end{array}$ & & & & Somatoform \\
\hline 306.59 & Psychogenic Gu Dis Nec & $\begin{array}{l}\text { Neurotic disorders, personality } \\
\text { disorders, and oth }\end{array}$ & $\mathrm{MH}$ & $\begin{array}{l}\text { Other Neurotic and } \\
\text { Nonpsychotic Mental } \\
\text { Disorders }\end{array}$ & & & & Somatoform \\
\hline 306.6 & Psychogen Endocrine Dis & $\begin{array}{l}\text { Neurotic disorders, personality } \\
\text { disorders, and oth }\end{array}$ & $\mathrm{MH}$ & $\begin{array}{l}\text { Other Neurotic and } \\
\text { Nonpsychotic Mental } \\
\text { Disorders }\end{array}$ & & & & Somatoform \\
\hline
\end{tabular}




\begin{tabular}{|c|c|c|c|c|c|c|c|c|}
\hline \multicolumn{9}{|c|}{ APPENDIX } \\
\hline diagn1 & Description & Subclass & MH SA & DPW_Diagcat & DSM_IV_TR & \begin{tabular}{c|} 
Abuse \\
Dependency
\end{tabular} & $\begin{array}{l}\text { Alcohol } \\
\text { Drug }\end{array}$ & Research_1A \\
\hline 306.7 & Psychogenic Sensory Dis & $\begin{array}{l}\text { Neurotic disorders, personality } \\
\text { disorders, and oth }\end{array}$ & $\mathrm{MH}$ & $\begin{array}{l}\text { Other Neurotic and } \\
\text { Nonpsychotic Mental } \\
\text { Disorders }\end{array}$ & & & & Somatoform \\
\hline 306.8 & Psychogenic Disorder Nec & $\begin{array}{l}\text { Neurotic disorders, personality } \\
\text { disorders, and oth }\end{array}$ & $\mathrm{MH}$ & $\begin{array}{l}\text { Other Neurotic and } \\
\text { Nonpsychotic Mental } \\
\text { Disorders }\end{array}$ & & & & Somatoform \\
\hline 306.9 & Psychogenic Disorder Nos & $\begin{array}{l}\text { Neurotic disorders, personality } \\
\text { disorders, and oth }\end{array}$ & $\mathrm{MH}$ & $\begin{array}{l}\text { Other Neurotic and } \\
\text { Nonpsychotic Mental } \\
\text { Disorders }\end{array}$ & & & & Somatoform \\
\hline 307 & $\begin{array}{l}\text { Special Symptoms Or Syndromes, } \\
\text { Not Elsewhere Classified }\end{array}$ & $\begin{array}{l}\text { Neurotic disorders, personality } \\
\text { disorders, and oth }\end{array}$ & MH & $\begin{array}{l}\text { Special Symptoms or } \\
\text { Syndromes, not elsewhere } \\
\text { classified }\end{array}$ & & & & Neurologic \\
\hline 307.0 & Stammering Stuttering & $\begin{array}{l}\text { Neurotic disorders, personality } \\
\text { disorders, and oth }\end{array}$ & $\mathrm{MH}$ & $\begin{array}{l}\text { Special Symptoms or } \\
\text { Syndromes, not elsewhere } \\
\text { classified }\end{array}$ & Stuttering & & & Neurologic \\
\hline 307.1 & Anorexia Nervosa & $\begin{array}{l}\text { Neurotic disorders, personality } \\
\text { disorders, and oth }\end{array}$ & MH & $\begin{array}{l}\text { Special Symptoms or } \\
\text { Syndromes, not elsewhere } \\
\text { classified }\end{array}$ & Anorexia Nervosa & & & Eating Disorder \\
\hline 307.2 & Tics & $\begin{array}{l}\text { Neurotic disorders, personality } \\
\text { disorders, and oth }\end{array}$ & $\mathrm{MH}$ & $\begin{array}{l}\text { Special Symptoms or } \\
\text { Syndromes, not elsewhere } \\
\text { classified }\end{array}$ & & & & Neurologic \\
\hline 307.20 & Tic Disorder Nos & $\begin{array}{l}\text { Neurotic disorders, personality } \\
\text { disorders, and oth }\end{array}$ & $\mathrm{MH}$ & $\begin{array}{l}\text { Special Symptoms or } \\
\text { Syndromes, not elsewhere } \\
\text { classified }\end{array}$ & Tic Disorder NOS & & & Neurologic \\
\hline 307.21 & Transient Tic, Childhood & $\begin{array}{l}\text { Neurotic disorders, personality } \\
\text { disorders, and oth }\end{array}$ & MH & $\begin{array}{l}\text { Special Symptoms or } \\
\text { Syndromes, not elsewhere } \\
\text { classified }\end{array}$ & Transient Tic Disorder & & & Neurologic \\
\hline 307.22 & Chronic Motor Tic Dis & $\begin{array}{l}\text { Neurotic disorders, personality } \\
\text { disorders, and oth }\end{array}$ & $\mathrm{MH}$ & $\begin{array}{l}\text { Special Symptoms or } \\
\text { Syndromes, not elsewhere } \\
\text { classified }\end{array}$ & $\begin{array}{l}\text { Chronic Motor or Vocal } \\
\text { Tic Disorder }\end{array}$ & & & Neurologic \\
\hline 307.23 & Gilles Tourette Disorder & $\begin{array}{l}\text { Neurotic disorders, personality } \\
\text { disorders, and oth }\end{array}$ & $\mathrm{MH}$ & $\begin{array}{l}\text { Special Symptoms or } \\
\text { Syndromes, not elsewhere } \\
\text { classified }\end{array}$ & Tourette's Disorder & & & Neurologic \\
\hline 307.3 & Stereotyped Movements & $\begin{array}{l}\text { Neurotic disorders, personality } \\
\text { disorders, and oth }\end{array}$ & $\mathrm{MH}$ & $\begin{array}{l}\text { Special Symptoms or } \\
\text { Syndromes, not elsewhere } \\
\text { classified }\end{array}$ & $\begin{array}{l}\text { Sterotypic Movement } \\
\text { Disorder }\end{array}$ & & & Neurologic \\
\hline 307.4 & $\begin{array}{l}\text { Specific Disorders Of Sleep Of } \\
\text { Nonorganic Origin }\end{array}$ & $\begin{array}{l}\text { Neurotic disorders, personality } \\
\text { disorders, and oth }\end{array}$ & $\mathrm{MH}$ & $\begin{array}{l}\text { Special Symptoms or } \\
\text { Syndromes, not elsewhere } \\
\text { classified }\end{array}$ & & & & Sleep Disorders \\
\hline 307.40 & Nonorganic Sleep Dis Nos & $\begin{array}{l}\text { Neurotic disorders, personality } \\
\text { disorders, and oth }\end{array}$ & MH & $\begin{array}{l}\text { Special Symptoms or } \\
\text { Syndromes, not elsewhere } \\
\text { classified }\end{array}$ & & & & Sleep Disorders \\
\hline 307.41 & Transient Insomnia & $\begin{array}{l}\text { Neurotic disorders, personality } \\
\text { disorders, and oth }\end{array}$ & MH & $\begin{array}{l}\text { Special Symptoms or } \\
\text { Syndromes, not elsewhere } \\
\text { classified }\end{array}$ & & & & Sleep Disorders \\
\hline 307.42 & Persistent Insomnia & $\begin{array}{l}\text { Neurotic disorders, personality } \\
\text { disorders, and oth }\end{array}$ & $\mathrm{MH}$ & $\begin{array}{l}\text { Special Symptoms or } \\
\text { Syndromes, not elsewhere } \\
\text { classified }\end{array}$ & $\begin{array}{l}\text { [Insomnia Related } \\
\text { to...[Indicate the Axis I or } \\
\text { Axis II Disorder]/Primary } \\
\text { Insomnia] }\end{array}$ & & & Sleep Disorders \\
\hline
\end{tabular}




\begin{tabular}{|c|c|c|c|c|c|c|c|c|}
\hline \multicolumn{9}{|c|}{ APPENDIX } \\
\hline diagn1 & Description & Subclass & MHSA & DPW_Diagcat & DSM_IV_TR & $\begin{array}{c}\text { Abuse } \\
\text { Dependency }\end{array}$ & $\begin{array}{l}\text { Alcohol } \\
\text { Drug }\end{array}$ & Research_1A \\
\hline 307.43 & Transient Hypersomnia & $\begin{array}{l}\text { Neurotic disorders, personality } \\
\text { disorders, and oth }\end{array}$ & $\mathrm{MH}$ & $\begin{array}{l}\text { Special Symptoms or } \\
\text { Syndromes, not elsewhere } \\
\text { classified }\end{array}$ & & & & Sleep Disorders \\
\hline 307.44 & Persistent Hypersomnia & $\begin{array}{l}\text { Neurotic disorders, personality } \\
\text { disorders, and oth }\end{array}$ & MH & $\begin{array}{l}\text { Special Symptoms or } \\
\text { Syndromes, not elsewhere } \\
\text { classified }\end{array}$ & $\begin{array}{l}\text { [Hypersomnia Related } \\
\text { to... [Indicate the Axis I or } \\
\text { Axis II Disorder]/Primary } \\
\text { Hypersomnia] }\end{array}$ & & & Sleep Disorders \\
\hline 307.45 & Disrupt Sleep-Wake Cycle & $\begin{array}{l}\text { Neurotic disorders, personality } \\
\text { disorders, and oth }\end{array}$ & $\mathrm{MH}$ & $\begin{array}{l}\text { Special Symptoms or } \\
\text { Syndromes, not elsewhere } \\
\text { classified }\end{array}$ & $\begin{array}{l}\text { Circadian Rhythm Sleep } \\
\text { Disorder }\end{array}$ & & & Sleep Disorders \\
\hline 307.46 & Somnambulism Nght Terror & $\begin{array}{l}\text { Neurotic disorders, personality } \\
\text { disorders, and oth }\end{array}$ & MH & $\begin{array}{l}\text { Special Symptoms or } \\
\text { Syndromes, not elsewhere } \\
\text { classified }\end{array}$ & $\begin{array}{l}\text { [Sleep Terror } \\
\text { Disorder/Sleepwalking } \\
\text { Disorder] }\end{array}$ & & & Sleep Disorders \\
\hline 307.47 & Sleep Stage Dysfunc Nec & $\begin{array}{l}\text { Neurotic disorders, personality } \\
\text { disorders, and oth }\end{array}$ & $\mathrm{MH}$ & $\begin{array}{l}\text { Special Symptoms or } \\
\text { Syndromes, not elsewhere } \\
\text { classified }\end{array}$ & $\begin{array}{l}\text { [Dyssomnia } \\
\text { NOS/Nightmare } \\
\text { Disorder/Parasomnia } \\
\text { NOS] }\end{array}$ & & & Sleep Disorders \\
\hline 307.48 & Repetit Sleep Intrusion & $\begin{array}{l}\text { Neurotic disorders, personality } \\
\text { disorders, and oth }\end{array}$ & $\mathrm{MH}$ & $\begin{array}{l}\text { Special Symptoms or } \\
\text { Syndromes, not elsewhere } \\
\text { classified }\end{array}$ & & & & Sleep Disorders \\
\hline 307.49 & Nonorganic Sleep Dis Nec & $\begin{array}{l}\text { Neurotic disorders, personality } \\
\text { disorders, and oth }\end{array}$ & $\mathrm{MH}$ & $\begin{array}{l}\text { Special Symptoms or } \\
\text { Syndromes, not elsewhere } \\
\text { classified }\end{array}$ & & & & Sleep Disorders \\
\hline 307.5 & $\begin{array}{l}\text { Other And Unspecified Disorders Of } \\
\text { Eating }\end{array}$ & $\begin{array}{l}\text { Neurotic disorders, personality } \\
\text { disorders, and oth }\end{array}$ & $\mathrm{MH}$ & $\begin{array}{l}\text { Special Symptoms or } \\
\text { Syndromes, not elsewhere } \\
\text { classified }\end{array}$ & & & & Eating Disorder \\
\hline 307.50 & Eating Disorder Nos & $\begin{array}{l}\text { Neurotic disorders, personality } \\
\text { disorders, and oth }\end{array}$ & $\mathrm{MH}$ & $\begin{array}{l}\text { Special Symptoms or } \\
\text { Syndromes, not elsewhere } \\
\text { classified }\end{array}$ & Eating Disorder NOS & & & Eating Disorder \\
\hline 307.51 & Bulimia & $\begin{array}{l}\text { Neurotic disorders, personality } \\
\text { disorders, and oth }\end{array}$ & MH & $\begin{array}{l}\text { Special Symptoms or } \\
\text { Syndromes, not elsewhere } \\
\text { classified }\end{array}$ & Bulimia Nervosa & & & Eating Disorder \\
\hline 307.52 & Pica & $\begin{array}{l}\text { Neurotic disorders, personality } \\
\text { disorders, and oth }\end{array}$ & MH & $\begin{array}{l}\text { Special Symptoms or } \\
\text { Syndromes, not elsewhere } \\
\text { classified }\end{array}$ & Pica & & & Eating Disorder \\
\hline 307.53 & Psychogenic Rumination & $\begin{array}{l}\text { Neurotic disorders, personality } \\
\text { disorders, and oth }\end{array}$ & $\mathrm{MH}$ & $\begin{array}{l}\text { Special Symptoms or } \\
\text { Syndromes, not elsewhere } \\
\text { classified }\end{array}$ & Rumniation Disorder & & & Eating Disorder \\
\hline 307.54 & Psychogenic Vomiting & $\begin{array}{l}\text { Neurotic disorders, personality } \\
\text { disorders, and oth }\end{array}$ & MH & $\begin{array}{l}\text { Special Symptoms or } \\
\text { Syndromes, not elsewhere } \\
\text { classified }\end{array}$ & & & & Eating Disorder \\
\hline 307.59 & Eating Disorder Nec & $\begin{array}{l}\text { Neurotic disorders, personality } \\
\text { disorders, and oth }\end{array}$ & $\mathrm{MH}$ & $\begin{array}{l}\text { Special Symptoms or } \\
\text { Syndromes, not elsewhere } \\
\text { classified }\end{array}$ & $\begin{array}{l}\text { Feeding Disorder of } \\
\text { Infancy or Early } \\
\text { Childhood }\end{array}$ & & & Eating Disorder \\
\hline 307.6 & Enuresis & $\begin{array}{l}\text { Neurotic disorders, personality } \\
\text { disorders, and oth }\end{array}$ & $\mathrm{MH}$ & $\begin{array}{l}\text { Special Symptoms or } \\
\text { Syndromes, not elsewhere } \\
\text { classified }\end{array}$ & $\begin{array}{l}\text { Enuresis (Not Due to a } \\
\text { General Medical } \\
\text { Condition) }\end{array}$ & & & Childhood Disorder \\
\hline
\end{tabular}




\begin{tabular}{|c|c|c|c|c|c|c|c|c|}
\hline \multicolumn{9}{|c|}{ APPENDIX } \\
\hline diagn1 & Description & Subclass & MH SA & DPW_Diagcat & DSM_IV_TR & \begin{tabular}{|c|} 
Abuse \\
Dependency \\
\end{tabular} & $\begin{array}{l}\text { Alcohol } \\
\text { Drug }\end{array}$ & Research_1A \\
\hline 307.7 & Encopresis & $\begin{array}{l}\text { Neurotic disorders, personality } \\
\text { disorders, and oth }\end{array}$ & $\mathrm{MH}$ & $\begin{array}{l}\text { Special Symptoms or } \\
\text { Syndromes, not elsewhere } \\
\text { classified }\end{array}$ & $\begin{array}{l}\text { Encopresis, Without } \\
\text { Constipation and } \\
\text { Overflow Incontinence }\end{array}$ & & & Childhood Disorder \\
\hline 307.8 & Psychalgia & $\begin{array}{l}\text { Neurotic disorders, personality } \\
\text { disorders, and oth }\end{array}$ & $\mathrm{MH}$ & $\begin{array}{l}\text { Special Symptoms or } \\
\text { Syndromes, not elsewhere } \\
\text { classified }\end{array}$ & & & & Somatoform \\
\hline 307.80 & Psychogenic Pain Nos & $\begin{array}{l}\text { Neurotic disorders, personality } \\
\text { disorders, and oth }\end{array}$ & $\mathrm{MH}$ & $\begin{array}{l}\text { Special Symptoms or } \\
\text { Syndromes, not elsewhere } \\
\text { classified }\end{array}$ & $\begin{array}{l}\text { Pain Disorder Associated } \\
\text { with Psychological } \\
\text { Factors }\end{array}$ & & & Somatoform \\
\hline 307.81 & Tension Headache & $\begin{array}{l}\text { Neurotic disorders, personality } \\
\text { disorders, and oth }\end{array}$ & $\mathrm{MH}$ & $\begin{array}{l}\text { Special Symptoms or } \\
\text { Syndromes, not elsewhere } \\
\text { classified }\end{array}$ & & & & Somatoform \\
\hline 307.89 & Psychogenic Pain Nec & $\begin{array}{l}\text { Neurotic disorders, personality } \\
\text { disorders, and oth }\end{array}$ & $\mathrm{MH}$ & $\begin{array}{l}\text { Special Symptoms or } \\
\text { Syndromes, not elsewhere } \\
\text { classified }\end{array}$ & $\begin{array}{l}\text { Pain Disorder Associated } \\
\text { with Both Psychological } \\
\text { Factors and a General } \\
\text { Medical Condition }\end{array}$ & & & Somatoform \\
\hline 307.9 & Special Symptom Nec Nos & $\begin{array}{l}\text { Neurotic disorders, personality } \\
\text { disorders, and oth }\end{array}$ & $\mathrm{MH}$ & $\begin{array}{l}\text { Special Symptoms or } \\
\text { Syndromes, not elsewhere } \\
\text { classified }\end{array}$ & $\begin{array}{l}\text { Communication Disorder } \\
\text { NOS }\end{array}$ & & & Developmental Delays \\
\hline 308 & Acute Reaction To Stress & $\begin{array}{l}\text { Neurotic disorders, personality } \\
\text { disorders, and oth }\end{array}$ & MH & Acute Reaction to Stress & & & & Anxiety \\
\hline 308.0 & Stress React, Emotional & $\begin{array}{l}\text { Neurotic disorders, personality } \\
\text { disorders, and oth }\end{array}$ & $\mathrm{MH}$ & Acute Reaction to Stress & & & & Anxiety \\
\hline 308.1 & Stress Reaction, Fugue & $\begin{array}{l}\text { Neurotic disorders, personality } \\
\text { disorders, and oth }\end{array}$ & $\mathrm{MH}$ & Acute Reaction to Stress & & & & Anxiety \\
\hline 308.2 & Stress React, Psychomot & $\begin{array}{l}\text { Neurotic disorders, personality } \\
\text { disorders, and oth }\end{array}$ & $\mathrm{MH}$ & Acute Reaction to Stress & & & & Anxiety \\
\hline 308.3 & Acute Stress React Nec & $\begin{array}{l}\text { Neurotic disorders, personality } \\
\text { disorders, and oth }\end{array}$ & $\mathrm{MH}$ & Acute Reaction to Stress & Acute Stress Disorder & & & Anxiety \\
\hline 308.4 & Stress React, Mixed Dis & $\begin{array}{l}\text { Neurotic disorders, personality } \\
\text { disorders, and oth }\end{array}$ & $\mathrm{MH}$ & Acute Reaction to Stress & & & & Anxiety \\
\hline 308.9 & Acute Stress React Nos & $\begin{array}{l}\text { Neurotic disorders, personality } \\
\text { disorders, and oth }\end{array}$ & $\mathrm{MH}$ & Acute Reaction to Stress & & & & Anxiety \\
\hline 309 & Adjustment Reaction & $\begin{array}{l}\text { Neurotic disorders, personality } \\
\text { disorders, and oth }\end{array}$ & $\mathrm{MH}$ & Adjustment Reaction & & & & Adjustment \\
\hline 309.0 & Brief Depressive React & $\begin{array}{l}\text { Neurotic disorders, personality } \\
\text { disorders, and oth }\end{array}$ & $\mathrm{MH}$ & Adjustment Reaction & $\begin{array}{l}\text { Adjustment Disorder with } \\
\text { Depressed Mood }\end{array}$ & & & Adjustment \\
\hline 309.00 & $\begin{array}{l}\text { Invalid Code }-309.0=\text { Brief } \\
\text { Depressive React }\end{array}$ & $\begin{array}{l}\text { Neurotic disorders, personality } \\
\text { disorders, and oth }\end{array}$ & $\mathrm{MH}$ & Adjustment Reaction & & & & Adjustment \\
\hline 309.1 & Prolong Depressive React & $\begin{array}{l}\text { Neurotic disorders, personality } \\
\text { disorders, and oth }\end{array}$ & $\mathrm{MH}$ & Adjustment Reaction & & & & Adjustment \\
\hline 309.2 & $\begin{array}{l}\text { With Predominant Disturbance Of } \\
\text { Other Emotions }\end{array}$ & $\begin{array}{l}\text { Neurotic disorders, personality } \\
\text { disorders, and oth }\end{array}$ & $\mathrm{MH}$ & Adjustment Reaction & & & & Adjustment \\
\hline 309.21 & Separation Anxiety & $\begin{array}{l}\text { Neurotic disorders, personality } \\
\text { disorders, and oth }\end{array}$ & $\mathrm{MH}$ & Adjustment Reaction & $\begin{array}{l}\text { Separation Anxiety } \\
\text { Disorder }\end{array}$ & & & Adjustment \\
\hline 309.22 & Emancipation Disorder & $\begin{array}{l}\text { Neurotic disorders, personality } \\
\text { disorders, and oth }\end{array}$ & $\mathrm{MH}$ & Adjustment Reaction & & & & Adjustment \\
\hline 309.23 & Academic Work Inhibition & $\begin{array}{l}\text { Neurotic disorders, personality } \\
\text { disorders, and oth }\end{array}$ & $\mathrm{MH}$ & Adjustment Reaction & & & & Adjustment \\
\hline
\end{tabular}




\begin{tabular}{|c|c|c|c|c|c|c|c|c|}
\hline \multicolumn{9}{|c|}{ APPENDIX } \\
\hline diagn1 & Description & Subclass & MH SA & DPW_Diagcat & DSM_IV_TR & $\begin{array}{c}\text { Abuse } \\
\text { Dependency }\end{array}$ & $\begin{array}{l}\text { Alcohol } \\
\text { Drug } \\
\end{array}$ & Research_1A \\
\hline 309.24 & Adj React-Anxious Mood & $\begin{array}{l}\text { Neurotic disorders, personality } \\
\text { disorders, and oth }\end{array}$ & MH & Adjustment Reaction & $\begin{array}{l}\text { Adjustment Disorder with } \\
\text { Anxiety }\end{array}$ & & & Adjustment \\
\hline 309.28 & Adj React-Mixed Emotion & $\begin{array}{l}\text { Neurotic disorders, personality } \\
\text { disorders, and oth }\end{array}$ & MH & Adjustment Reaction & $\begin{array}{l}\text { Adjustment Disorder with } \\
\text { Mixed Anxiety and } \\
\text { Depressed Mood }\end{array}$ & & & Adjustment \\
\hline 309.29 & Adj React-Emotion Nec & $\begin{array}{l}\text { Neurotic disorders, personality } \\
\text { disorders, and oth }\end{array}$ & $\mathrm{MH}$ & Adjustment Reaction & & & & Adjustment \\
\hline 309.3 & Adjust React-Conduct Dis & $\begin{array}{l}\text { Neurotic disorders, personality } \\
\text { disorders, and oth }\end{array}$ & $\mathrm{MH}$ & Adjustment Reaction & $\begin{array}{l}\text { Adjustment Disorder with } \\
\text { Disturbance of Conduct }\end{array}$ & & & Adjustment \\
\hline 309.30 & $\begin{array}{l}\text { Invalid Code - } 309.3=\text { Adjust React- } \\
\text { Conduct Dis }\end{array}$ & $\begin{array}{l}\text { Neurotic disorders, personality } \\
\text { disorders, and oth }\end{array}$ & $\mathrm{MH}$ & Adjustment Reaction & & & & Adjustment \\
\hline 309.4 & Adj React-Emotion Conduc & $\begin{array}{l}\text { Neurotic disorders, personality } \\
\text { disorders, and oth }\end{array}$ & $\mathrm{MH}$ & Adjustment Reaction & $\begin{array}{l}\text { Adjustment Disorder with } \\
\text { Mixed Disturbance of } \\
\text { Emotions and Conduct }\end{array}$ & & & Adjustment \\
\hline 309.40 & $\begin{array}{l}\text { Invalid Code }-309.4=\text { Adj React- } \\
\text { Emotion Conduc }\end{array}$ & $\begin{array}{l}\text { Neurotic disorders, personality } \\
\text { disorders, and oth }\end{array}$ & $\mathrm{MH}$ & Adjustment Reaction & & & & Adjustment \\
\hline 309.8 & $\begin{array}{l}\text { Other Specified Adjustment } \\
\text { Reactions }\end{array}$ & $\begin{array}{l}\text { Neurotic disorders, personality } \\
\text { disorders, and oth }\end{array}$ & MH & Adjustment Reaction & & & & Adjustment \\
\hline 309.81 & Prolong Posttraum Stress & $\begin{array}{l}\text { Neurotic disorders, personality } \\
\text { disorders, and oth }\end{array}$ & $\mathrm{MH}$ & Adjustment Reaction & $\begin{array}{l}\text { Posttraumatic Stress } \\
\text { Disorder }\end{array}$ & & & PTSD \\
\hline 309.82 & Adjust React-Phys Sympt & $\begin{array}{l}\text { Neurotic disorders, personality } \\
\text { disorders, and oth }\end{array}$ & $\mathrm{MH}$ & Adjustment Reaction & & & & Adjustment \\
\hline 309.83 & Adjust React-Withdrawal & $\begin{array}{l}\text { Neurotic disorders, personality } \\
\text { disorders, and oth }\end{array}$ & $\mathrm{MH}$ & Adjustment Reaction & & & & Adjustment \\
\hline 309.89 & Adjustment Reaction $\mathrm{Nec}$ & $\begin{array}{l}\text { Neurotic disorders, personality } \\
\text { disorders, and oth }\end{array}$ & MH & Adjustment Reaction & & & & Adjustment \\
\hline 309.9 & Adjustment Reaction Nos & $\begin{array}{l}\text { Neurotic disorders, personality } \\
\text { disorders, and oth }\end{array}$ & $\mathrm{MH}$ & Adjustment Reaction & $\begin{array}{l}\text { Adjustment Disorder } \\
\text { Unspecified }\end{array}$ & & & Adjustment \\
\hline 310 & $\begin{array}{l}\text { Specific Nonpsychotic Mental } \\
\text { Disorders Due To Organic Brain } \\
\text { Damage }\end{array}$ & $\begin{array}{l}\text { Neurotic disorders, personality } \\
\text { disorders, and oth }\end{array}$ & MH & $\begin{array}{l}\text { Other Neurotic and } \\
\text { Nonpsychotic Mental } \\
\text { Disorders }\end{array}$ & & & & $\begin{array}{l}\text { General Medical } \\
\text { Condition }\end{array}$ \\
\hline 310.0 & Frontal Lobe Syndrome & $\begin{array}{l}\text { Neurotic disorders, personality } \\
\text { disorders, and oth }\end{array}$ & MH & $\begin{array}{l}\text { Other Neurotic and } \\
\text { Nonpsychotic Mental } \\
\text { Disorders }\end{array}$ & & & & $\begin{array}{l}\text { General Medical } \\
\text { Condition }\end{array}$ \\
\hline 310.1 & Organic Personality Synd & $\begin{array}{l}\text { Neurotic disorders, personality } \\
\text { disorders, and oth }\end{array}$ & $\mathrm{MH}$ & $\begin{array}{l}\text { Other Neurotic and } \\
\text { Nonpsychotic Mental } \\
\text { Disorders }\end{array}$ & $\begin{array}{l}\text { Personality Change Due } \\
\text { to ... [Indicate the } \\
\text { General Medical } \\
\text { Condition] }\end{array}$ & & & $\begin{array}{l}\text { General Medical } \\
\text { Condition }\end{array}$ \\
\hline 310.2 & Postconcussion Syndrome & $\begin{array}{l}\text { Neurotic disorders, personality } \\
\text { disorders, and oth }\end{array}$ & $\mathrm{MH}$ & $\begin{array}{l}\text { Other Neurotic and } \\
\text { Nonpsychotic Mental } \\
\text { Disorders }\end{array}$ & & & & $\begin{array}{l}\text { General Medical } \\
\text { Condition }\end{array}$ \\
\hline 310.8 & Nonpsychot Brain Syn Nec & $\begin{array}{l}\text { Neurotic disorders, personality } \\
\text { disorders, and oth }\end{array}$ & $\mathrm{MH}$ & $\begin{array}{l}\text { Other Neurotic and } \\
\text { Nonpsychotic Mental } \\
\text { Disorders }\end{array}$ & & & & $\begin{array}{l}\text { General Medical } \\
\text { Condition }\end{array}$ \\
\hline 310.9 & Nonpsychot Brain Syn Nos & $\begin{array}{l}\text { Neurotic disorders, personality } \\
\text { disorders, and oth }\end{array}$ & $\mathrm{MH}$ & $\begin{array}{l}\text { Other Neurotic and } \\
\text { Nonpsychotic Mental } \\
\text { Disorders }\end{array}$ & & & & $\begin{array}{l}\text { General Medical } \\
\text { Condition }\end{array}$ \\
\hline
\end{tabular}


Measurement of Metabolic Monitoring in Youth and Adult Medicaid Recipients Prescribed Antipsychotics

APPENDIX Crosswalk of Diagnostic Categories Between State Categories (DPW) to DSM-IV-TR to Research-IA Categories Used by Community Care Behavioral Health Organization (continued)

\begin{tabular}{|c|c|c|c|c|c|c|c|c|}
\hline diagn1 & Description & Subclass & MH SA & DPW_Diagcat & DSM_IV_TR & $\begin{array}{c}\text { Abuse } \\
\text { Dependency }\end{array}$ & $\begin{array}{l}\text { Alcohol } \\
\text { Drug }\end{array}$ & Research_1A \\
\hline 311 & Depressive Disorder $\mathrm{Nec}$ & $\begin{array}{l}\text { Neurotic disorders, personality } \\
\text { disorders, and oth }\end{array}$ & $\mathrm{MH}$ & Depressive Disorder, NOS & $\begin{array}{l}\text { Depressive Disorder } \\
\text { NOS }\end{array}$ & & & $\begin{array}{l}\text { Dysthimia/Depression } \\
\text { NOS }\end{array}$ \\
\hline 311.0 & $\begin{array}{l}\text { Invalid Code - } 311 \text { = Depressive } \\
\text { Disorder Nec }\end{array}$ & $\begin{array}{l}\text { Neurotic disorders, personality } \\
\text { disorders, and oth }\end{array}$ & $\mathrm{MH}$ & Depressive Disorder, NOS & & & & $\begin{array}{l}\text { Dysthimia/Depression } \\
\text { NOS }\end{array}$ \\
\hline 311.00 & $\begin{array}{l}\text { Invalid Code - } 311 \text { = Depressive } \\
\text { Disorder Nec }\end{array}$ & $\begin{array}{l}\text { Neurotic disorders, personality } \\
\text { disorders, and oth }\end{array}$ & $\mathrm{MH}$ & Depressive Disorder, NOS & & & & $\begin{array}{l}\text { Dysthimia/Depression } \\
\text { NOS }\end{array}$ \\
\hline 312 & $\begin{array}{l}\text { Disturbance Of Conduct, Not } \\
\text { Elsewhere Classified }\end{array}$ & $\begin{array}{l}\text { Neurotic disorders, personality } \\
\text { disorders, and oth }\end{array}$ & $\mathrm{MH}$ & $\begin{array}{l}\text { Disturbance of Conduct, not } \\
\text { elsewhere classified }\end{array}$ & & & & Conduct \\
\hline 312.0 & $\begin{array}{l}\text { Undersocialized Conduct Disorder, } \\
\text { Aggressive Type }\end{array}$ & $\begin{array}{l}\text { Neurotic disorders, personality } \\
\text { disorders, and oth }\end{array}$ & $\mathrm{MH}$ & $\begin{array}{l}\text { Disturbance of Conduct, not } \\
\text { elsewhere classified }\end{array}$ & & & & Conduct \\
\hline 312.00 & Unsocial Aggress-Unspec & $\begin{array}{l}\text { Neurotic disorders, personality } \\
\text { disorders, and oth }\end{array}$ & $\mathrm{MH}$ & $\begin{array}{l}\text { Disturbance of Conduct, not } \\
\text { elsewhere classified }\end{array}$ & & & & Conduct \\
\hline 312.01 & Unsocial Aggression-Mild & $\begin{array}{l}\text { Neurotic disorders, personality } \\
\text { disorders, and oth }\end{array}$ & $\mathrm{MH}$ & $\begin{array}{l}\text { Disturbance of Conduct, not } \\
\text { elsewhere classified }\end{array}$ & & & & Conduct \\
\hline 312.02 & Unsocial Aggression-Mod & $\begin{array}{l}\text { Neurotic disorders, personality } \\
\text { disorders, and oth }\end{array}$ & $\mathrm{MH}$ & $\begin{array}{l}\text { Disturbance of Conduct, not } \\
\text { elsewhere classified }\end{array}$ & & & & Conduct \\
\hline 312.03 & Unsocial Aggress-Severe & $\begin{array}{l}\text { Neurotic disorders, personality } \\
\text { disorders, and oth }\end{array}$ & $\mathrm{MH}$ & $\begin{array}{l}\text { Disturbance of Conduct, not } \\
\text { elsewhere classified }\end{array}$ & & & & Conduct \\
\hline 312.1 & $\begin{array}{l}\text { Undersocialized Conduct Disorder, } \\
\text { Unaggressive Type }\end{array}$ & $\begin{array}{l}\text { Neurotic disorders, personality } \\
\text { disorders, and oth }\end{array}$ & $\mathrm{MH}$ & $\begin{array}{l}\text { Disturbance of Conduct, not } \\
\text { elsewhere classified }\end{array}$ & & & & Conduct \\
\hline 312.10 & Unsocial Unaggress-Unsp & $\begin{array}{l}\text { Neurotic disorders, personality } \\
\text { disorders, and oth }\end{array}$ & $\mathrm{MH}$ & $\begin{array}{l}\text { Disturbance of Conduct, not } \\
\text { elsewhere classified }\end{array}$ & & & & Conduct \\
\hline 312.11 & Unsocial Unaggress-Mild & $\begin{array}{l}\text { Neurotic disorders, personality } \\
\text { disorders, and oth }\end{array}$ & $\mathrm{MH}$ & $\begin{array}{l}\text { Disturbance of Conduct, not } \\
\text { elsewhere classified }\end{array}$ & & & & Conduct \\
\hline 312.12 & Unsocial Unaggress-Mod & $\begin{array}{l}\text { Neurotic disorders, personality } \\
\text { disorders, and oth }\end{array}$ & $\mathrm{MH}$ & $\begin{array}{l}\text { Disturbance of Conduct, not } \\
\text { elsewhere classified }\end{array}$ & & & & Conduct \\
\hline 312.13 & Unsocial Unaggr-Severe & $\begin{array}{l}\text { Neurotic disorders, personality } \\
\text { disorders, and oth }\end{array}$ & $\mathrm{MH}$ & $\begin{array}{l}\text { Disturbance of Conduct, not } \\
\text { elsewhere classified }\end{array}$ & & & & Conduct \\
\hline 312.2 & Socialized Conduct Disorder & $\begin{array}{l}\text { Neurotic disorders, personality } \\
\text { disorders, and oth }\end{array}$ & $\mathrm{MH}$ & $\begin{array}{l}\text { Disturbance of Conduct, not } \\
\text { elsewhere classified }\end{array}$ & & & & Conduct \\
\hline 312.20 & Social Conduct Dis-Unsp & $\begin{array}{l}\text { Neurotic disorders, personality } \\
\text { disorders, and oth }\end{array}$ & MH & $\begin{array}{l}\text { Disturbance of Conduct, not } \\
\text { elsewhere classified }\end{array}$ & & & & Conduct \\
\hline 312.21 & Social Conduct Dis-Mild & $\begin{array}{l}\text { Neurotic disorders, personality } \\
\text { disorders, and oth }\end{array}$ & MH & $\begin{array}{l}\text { Disturbance of Conduct, not } \\
\text { elsewhere classified }\end{array}$ & & & & Conduct \\
\hline 312.22 & Social Conduct Dis-Mod & $\begin{array}{l}\text { Neurotic disorders, personality } \\
\text { disorders, and oth }\end{array}$ & $\mathrm{MH}$ & $\begin{array}{l}\text { Disturbance of Conduct, not } \\
\text { elsewhere classified }\end{array}$ & & & & Conduct \\
\hline 312.23 & Social Conduct Dis-Sev & $\begin{array}{l}\text { Neurotic disorders, personality } \\
\text { disorders, and oth }\end{array}$ & $\mathrm{MH}$ & $\begin{array}{l}\text { Disturbance of Conduct, not } \\
\text { elsewhere classified }\end{array}$ & & & & Conduct \\
\hline 312.3 & $\begin{array}{l}\text { Disorders Of Impulse Control, Not } \\
\text { Elsewhere Classified }\end{array}$ & $\begin{array}{l}\text { Neurotic disorders, personality } \\
\text { disorders, and oth }\end{array}$ & $\mathrm{MH}$ & $\begin{array}{l}\text { Disturbance of Conduct, not } \\
\text { elsewhere classified }\end{array}$ & & & & Impulse \\
\hline 312.30 & Impulse Control Dis Nos & $\begin{array}{l}\text { Neurotic disorders, personality } \\
\text { disorders, and oth }\end{array}$ & $\mathrm{MH}$ & $\begin{array}{l}\text { Disturbance of Conduct, not } \\
\text { elsewhere classified }\end{array}$ & $\begin{array}{l}\text { Impulse-Control Disorder } \\
\text { NOS }\end{array}$ & & & Impulse \\
\hline 312.31 & Pathological Gambling & $\begin{array}{l}\text { Neurotic disorders, personality } \\
\text { disorders, and oth }\end{array}$ & $\mathrm{MH}$ & $\begin{array}{l}\text { Disturbance of Conduct, not } \\
\text { elsewhere classified }\end{array}$ & Pathological Gambling & & & Impulse \\
\hline 312.32 & Kleptomania & $\begin{array}{l}\text { Neurotic disorders, personality } \\
\text { disorders, and oth }\end{array}$ & $\mathrm{MH}$ & $\begin{array}{l}\text { Disturbance of Conduct, not } \\
\text { elsewhere classified }\end{array}$ & Kleptomania & & & Impulse \\
\hline 312.33 & Pyromania & $\begin{array}{l}\text { Neurotic disorders, personality } \\
\text { disorders, and oth }\end{array}$ & $\mathrm{MH}$ & $\begin{array}{l}\text { Disturbance of Conduct, not } \\
\text { elsewhere classified }\end{array}$ & Pyromania & & & Impulse \\
\hline 312.34 & Intermitt Explosive Dis & $\begin{array}{l}\text { Neurotic disorders, personality } \\
\text { disorders, and oth }\end{array}$ & $\mathrm{MH}$ & $\begin{array}{l}\text { Disturbance of Conduct, not } \\
\text { elsewhere classified }\end{array}$ & $\begin{array}{l}\text { Intermittent Explosive } \\
\text { Disorder }\end{array}$ & & & Impulse \\
\hline
\end{tabular}


APPENDIX Crosswalk of Diagnostic Categories Between State Categories (DPW) to DSM-IV-TR to Research-IA Categories Used by Community Care Behavioral Health Organization (continued)

\begin{tabular}{|c|c|c|c|c|c|c|c|c|}
\hline diagn1 & Description & Subclass & MH SA & DPW_Diagcat & DSM_IV_TR & $\begin{array}{c}\text { Abuse } \\
\text { Dependency }\end{array}$ & $\begin{array}{l}\text { Alcohol } \\
\text { Drug }\end{array}$ & Research_1A \\
\hline 312.35 & Isolated Explosive Dis & $\begin{array}{l}\text { Neurotic disorders, personality } \\
\text { disorders, and oth }\end{array}$ & $\mathrm{MH}$ & $\begin{array}{l}\text { Disturbance of Conduct, not } \\
\text { elsewhere classified }\end{array}$ & & & & Impulse \\
\hline 312.39 & Impulse Control Dis $\mathrm{Nec}$ & $\begin{array}{l}\text { Neurotic disorders, personality } \\
\text { disorders, and oth }\end{array}$ & $\mathrm{MH}$ & $\begin{array}{l}\text { Disturbance of Conduct, not } \\
\text { elsewhere classified }\end{array}$ & Trichotillomania & & & Impulse \\
\hline 312.4 & Mix Dis Conduct Emotion & $\begin{array}{l}\text { Neurotic disorders, personality } \\
\text { disorders, and oth }\end{array}$ & $\mathrm{MH}$ & $\begin{array}{l}\text { Disturbance of Conduct, not } \\
\text { elsewhere classified }\end{array}$ & & & & Conduct \\
\hline 312.40 & $\begin{array}{l}\text { Invalid Code }-312.4=\text { Mix Dis } \\
\text { Conduct Emotion }\end{array}$ & $\begin{array}{l}\text { Neurotic disorders, personality } \\
\text { disorders, and oth }\end{array}$ & $\mathrm{MH}$ & $\begin{array}{l}\text { Disturbance of Conduct, not } \\
\text { elsewhere classified }\end{array}$ & & & & Conduct \\
\hline 312.8 & $\begin{array}{l}\text { Other Specified Disturbances Of } \\
\text { Conduct, Not Elsewhere Classified }\end{array}$ & $\begin{array}{l}\text { Neurotic disorders, personality } \\
\text { disorders, and oth }\end{array}$ & MH & $\begin{array}{l}\text { Disturbance of Conduct, not } \\
\text { elsewhere classified }\end{array}$ & & & & Conduct \\
\hline 312.81 & Cndct Dsrdr Chldhd Onst & $\begin{array}{l}\text { Neurotic disorders, personality } \\
\text { disorders, and oth }\end{array}$ & $\mathrm{MH}$ & $\begin{array}{l}\text { Disturbance of Conduct, not } \\
\text { elsewhere classified }\end{array}$ & $\begin{array}{l}\text { Conduct Disorder, } \\
\text { Childhood-Onset Type }\end{array}$ & & & Conduct \\
\hline 312.82 & Cndct Dsrdr Adlscnt Onst & $\begin{array}{l}\text { Neurotic disorders, personality } \\
\text { disorders, and oth }\end{array}$ & $\mathrm{MH}$ & $\begin{array}{l}\text { Disturbance of Conduct, not } \\
\text { elsewhere classified }\end{array}$ & $\begin{array}{l}\text { Conduct Disorder, } \\
\text { Adolescent-Onset Type }\end{array}$ & & & Conduct \\
\hline 312.89 & Other Conduct Disorder & $\begin{array}{l}\text { Neurotic disorders, personality } \\
\text { disorders, and oth }\end{array}$ & $\mathrm{MH}$ & $\begin{array}{l}\text { Disturbance of Conduct, not } \\
\text { elsewhere classified }\end{array}$ & $\begin{array}{l}\text { Conduct Disorder, } \\
\text { Unspecified Onset }\end{array}$ & & & Conduct \\
\hline 312.9 & Conduct Disturbance Nos & $\begin{array}{l}\text { Neurotic disorders, personality } \\
\text { disorders, and oth }\end{array}$ & $\mathrm{MH}$ & $\begin{array}{l}\text { Disturbance of Conduct, not } \\
\text { elsewhere classified }\end{array}$ & $\begin{array}{l}\text { Disruptive Behavior } \\
\text { Disorder NOS }\end{array}$ & & & Conduct \\
\hline 312.90 & $\begin{array}{l}\text { Invalid Code }-312.9=\text { Conduct } \\
\text { Disturbance Nos }\end{array}$ & $\begin{array}{l}\text { Neurotic disorders, personality } \\
\text { disorders, and oth }\end{array}$ & $\mathrm{MH}$ & $\begin{array}{l}\text { Disturbance of Conduct, not } \\
\text { elsewhere classified }\end{array}$ & & & & Conduct \\
\hline 313 & $\begin{array}{l}\text { Disturbance Of Emotions Specific } \\
\text { To Childhood And Adolescence }\end{array}$ & $\begin{array}{l}\text { Neurotic disorders, personality } \\
\text { disorders, and oth }\end{array}$ & MH & $\begin{array}{l}\text { Disturbance of Emotions } \\
\text { Specific to Childhood \& } \\
\text { Adolescence }\end{array}$ & & & & Childhood Disorder \\
\hline 313.0 & Overanxious Disorder & $\begin{array}{l}\text { Neurotic disorders, personality } \\
\text { disorders, and oth }\end{array}$ & MH & $\begin{array}{l}\text { Disturbance of Emotions } \\
\text { Specific to Childhood \& } \\
\text { Adolescence }\end{array}$ & & & & Childhood Disorder \\
\hline 313.1 & Misery Unhappiness Dis & $\begin{array}{l}\text { Neurotic disorders, personality } \\
\text { disorders, and oth }\end{array}$ & $\mathrm{MH}$ & $\begin{array}{l}\text { Disturbance of Emotions } \\
\text { Specific to Childhood \& } \\
\text { Adolescence }\end{array}$ & & & & Childhood Disorder \\
\hline 313.2 & $\begin{array}{l}\text { Sensitivity, Shyness, And Social } \\
\text { Withdrawal Disorder }\end{array}$ & $\begin{array}{l}\text { Neurotic disorders, personality } \\
\text { disorders, and oth }\end{array}$ & $\mathrm{MH}$ & $\begin{array}{l}\text { Disturbance of Emotions } \\
\text { Specific to Childhood \& } \\
\text { Adolescence }\end{array}$ & & & & Childhood Disorder \\
\hline 313.21 & Shyness Disorder-Child & $\begin{array}{l}\text { Neurotic disorders, personality } \\
\text { disorders, and oth }\end{array}$ & MH & $\begin{array}{l}\text { Disturbance of Emotions } \\
\text { Specific to Childhood \& } \\
\text { Adolescence }\end{array}$ & & & & Childhood Disorder \\
\hline 313.22 & Introverted Dis-Child & $\begin{array}{l}\text { Neurotic disorders, personality } \\
\text { disorders, and oth }\end{array}$ & $\mathrm{MH}$ & $\begin{array}{l}\text { Disturbance of Emotions } \\
\text { Specific to Childhood \& } \\
\text { Adolescence }\end{array}$ & & & & Childhood Disorder \\
\hline 313.23 & Elective Mutism & $\begin{array}{l}\text { Neurotic disorders, personality } \\
\text { disorders, and oth }\end{array}$ & $\mathrm{MH}$ & $\begin{array}{l}\text { Disturbance of Emotions } \\
\text { Specific to Childhood \& } \\
\text { Adolescence }\end{array}$ & Selective Mutism & & & Childhood Disorder \\
\hline 313.3 & Relationship Problems & $\begin{array}{l}\text { Neurotic disorders, personality } \\
\text { disorders, and oth }\end{array}$ & MH & $\begin{array}{l}\text { Disturbance of Emotions } \\
\text { Specific to Childhood \& } \\
\text { Adolescence }\end{array}$ & & & & Childhood Disorder \\
\hline 313.8 & $\begin{array}{l}\text { Other Or Mixed Emotional } \\
\text { Disturbances Of Childhood Or } \\
\text { Adolescence }\end{array}$ & $\begin{array}{l}\text { Neurotic disorders, personality } \\
\text { disorders, and oth }\end{array}$ & $\mathrm{MH}$ & $\begin{array}{l}\text { Disturbance of Emotions } \\
\text { Specific to Childhood \& } \\
\text { Adolescence }\end{array}$ & & & & Childhood Disorder \\
\hline 313.81 & Oppositional Disorder & $\begin{array}{l}\text { Neurotic disorders, personality } \\
\text { disorders, and oth }\end{array}$ & $\mathrm{MH}$ & $\begin{array}{l}\text { Disturbance of Emotions } \\
\text { Specific to Childhood \& } \\
\text { Adolescence }\end{array}$ & $\begin{array}{l}\text { Oppositional Defiant } \\
\text { Disorder }\end{array}$ & & & ODD \\
\hline
\end{tabular}




\begin{tabular}{|c|c|c|c|c|c|c|c|c|}
\hline \multicolumn{9}{|c|}{ APPENDIX } \\
\hline diagn1 & Description & Subclass & MH SA & DPW_Diagcat & DSM_IV_TR & $\begin{array}{c}\text { Abuse } \\
\text { Dependency }\end{array}$ & $\begin{array}{l}\text { Alcohol } \\
\text { Drug }\end{array}$ & Research_1A \\
\hline 313.82 & Identity Disorder & $\begin{array}{l}\text { Neurotic disorders, personality } \\
\text { disorders, and oth }\end{array}$ & MH & $\begin{array}{l}\text { Disturbance of Emotions } \\
\text { Specific to Childhood \& } \\
\text { Adolescence }\end{array}$ & Identity Problem & & & Childhood Disorder \\
\hline 313.83 & Academic Underachievment & $\begin{array}{l}\text { Neurotic disorders, personality } \\
\text { disorders, and oth }\end{array}$ & $\mathrm{MH}$ & $\begin{array}{l}\text { Disturbance of Emotions } \\
\text { Specific to Childhood \& } \\
\text { Adolescence }\end{array}$ & & & & Childhood Disorder \\
\hline 313.89 & Emotional Dis Child $\mathrm{Nec}$ & $\begin{array}{l}\text { Neurotic disorders, personality } \\
\text { disorders, and oth }\end{array}$ & $\mathrm{MH}$ & $\begin{array}{l}\text { Disturbance of Emotions } \\
\text { Specific to Childhood \& } \\
\text { Adolescence }\end{array}$ & $\begin{array}{l}\text { Reactive Attachment } \\
\text { Disorder of Infancy or } \\
\text { Early Childhood }\end{array}$ & & & Childhood Disorder \\
\hline 313.9 & Emotional Dis Child Nos & $\begin{array}{l}\text { Neurotic disorders, personality } \\
\text { disorders, and oth }\end{array}$ & $\mathrm{MH}$ & $\begin{array}{l}\text { Disturbance of Emotions } \\
\text { Specific to Childhood \& } \\
\text { Adolescence }\end{array}$ & $\begin{array}{l}\text { Disorder of Infancy, } \\
\text { Childhood, or } \\
\text { Adolescence NOS }\end{array}$ & & & Childhood Disorder \\
\hline 314 & $\begin{array}{l}\text { Hyperkinetic Syndrome Of } \\
\text { Childhood }\end{array}$ & $\begin{array}{l}\text { Neurotic disorders, personality } \\
\text { disorders, and oth }\end{array}$ & $\mathrm{MH}$ & $\begin{array}{l}\text { Hyperkinetic Syndrome of } \\
\text { Childhood }\end{array}$ & & & & ADHD \\
\hline 314.0 & Attention Deficit Disorder & $\begin{array}{l}\text { Neurotic disorders, personality } \\
\text { disorders, and oth }\end{array}$ & $\mathrm{MH}$ & $\begin{array}{l}\text { Hyperkinetic Syndrome of } \\
\text { Childhood }\end{array}$ & & & & ADHD \\
\hline 314.00 & Attn Defic Nonhyperact & $\begin{array}{l}\text { Neurotic disorders, personality } \\
\text { disorders, and oth }\end{array}$ & $\mathrm{MH}$ & $\begin{array}{l}\text { Hyperkinetic Syndrome of } \\
\text { Childhood }\end{array}$ & $\begin{array}{l}\text { Attention- } \\
\text { Deficit/Hyperactivity } \\
\text { Disorder, Predominantly } \\
\text { Inattentive Type }\end{array}$ & & & ADHD \\
\hline 314.01 & Attn Deficit W Hyperact & $\begin{array}{l}\text { Neurotic disorders, personality } \\
\text { disorders, and oth }\end{array}$ & $\mathrm{MH}$ & $\begin{array}{l}\text { Hyperkinetic Syndrome of } \\
\text { Childhood }\end{array}$ & $\begin{array}{l}\text { Attention- } \\
\text { Deficit/Hyperactivity } \\
\text { Disorder [Combined } \\
\text { Type/ Predominantly } \\
\text { Hyperactive-Impulsive } \\
\text { Type] }\end{array}$ & & & ADHD \\
\hline 314.1 & Hyperkinet W Devel Delay & $\begin{array}{l}\text { Neurotic disorders, personality } \\
\text { disorders, and oth }\end{array}$ & $\mathrm{MH}$ & $\begin{array}{l}\text { Hyperkinetic Syndrome of } \\
\text { Childhood }\end{array}$ & & & & ADHD \\
\hline 314.2 & Hyperkinetic Conduct Dis & $\begin{array}{l}\text { Neurotic disorders, personality } \\
\text { disorders, and oth }\end{array}$ & $\mathrm{MH}$ & $\begin{array}{l}\text { Hyperkinetic Syndrome of } \\
\text { Childhood }\end{array}$ & & & & ADHD \\
\hline 314.8 & Other Hyperkinetic Synd & $\begin{array}{l}\text { Neurotic disorders, personality } \\
\text { disorders, and oth }\end{array}$ & $\mathrm{MH}$ & $\begin{array}{l}\text { Hyperkinetic Syndrome of } \\
\text { Childhood }\end{array}$ & & & & ADHD \\
\hline 314.9 & Hyperkinetic Synd Nos & $\begin{array}{l}\text { Neurotic disorders, personality } \\
\text { disorders, and oth }\end{array}$ & $\mathrm{MH}$ & $\begin{array}{l}\text { Hyperkinetic Syndrome of } \\
\text { Childhood }\end{array}$ & $\begin{array}{l}\text { Attention- } \\
\text { Deficit/Hyperactivity } \\
\text { Disorder NOS }\end{array}$ & & & ADHD \\
\hline 314.90 & $\begin{array}{l}\text { Invalid Code - } 314.9=\text { Hyperkinetic } \\
\text { Synd Nos }\end{array}$ & $\begin{array}{l}\text { Neurotic disorders, personality } \\
\text { disorders, and oth }\end{array}$ & MH & $\begin{array}{l}\text { Hyperkinetic Syndrome of } \\
\text { Childhood }\end{array}$ & & & & ADHD \\
\hline 315 & Specific Delays In Development & $\begin{array}{l}\text { Neurotic disorders, personality } \\
\text { disorders, and oth }\end{array}$ & $\mathrm{MH}$ & $\begin{array}{l}\text { Specific Delays in } \\
\text { Development }\end{array}$ & & & & Developmental Delays \\
\hline 315.0 & $\begin{array}{l}\text { Invalid Code - } 315=\text { Specific Delays } \\
\text { In Development }\end{array}$ & $\begin{array}{l}\text { Neurotic disorders, personality } \\
\text { disorders, and oth }\end{array}$ & $\mathrm{MH}$ & $\begin{array}{l}\text { Specific Delays in } \\
\text { Development }\end{array}$ & & & & Developmental Delays \\
\hline 315.00 & Reading Disorder Nos & $\begin{array}{l}\text { Neurotic disorders, personality } \\
\text { disorders, and oth }\end{array}$ & $\mathrm{MH}$ & $\begin{array}{l}\text { Specific Delays in } \\
\text { Development }\end{array}$ & Reading Disorder & & & Developmental Delays \\
\hline 315.01 & Alexia & $\begin{array}{l}\text { Neurotic disorders, personality } \\
\text { disorders, and oth }\end{array}$ & $\mathrm{MH}$ & $\begin{array}{l}\text { Specific Delays in } \\
\text { Development }\end{array}$ & & & & Developmental Delays \\
\hline 315.02 & Developmental Dyslexia & $\begin{array}{l}\text { Neurotic disorders, personality } \\
\text { disorders, and oth }\end{array}$ & $\mathrm{MH}$ & $\begin{array}{l}\text { Specific Delays in } \\
\text { Development }\end{array}$ & & & & Developmental Delays \\
\hline 315.09 & Reading Disorder $\mathrm{Nec}$ & $\begin{array}{l}\text { Neurotic disorders, personality } \\
\text { disorders, and oth }\end{array}$ & $\mathrm{MH}$ & $\begin{array}{l}\text { Specific Delays in } \\
\text { Development }\end{array}$ & & & & Developmental Delays \\
\hline
\end{tabular}




\begin{tabular}{|c|c|c|c|c|c|c|c|c|}
\hline \multicolumn{9}{|c|}{ APPENDIX } \\
\hline diagn1 & Description & Subclass & MH SA & DPW_Diagcat & DSM_IV_TR & \begin{tabular}{|c|} 
Abuse \\
Dependency
\end{tabular} & \begin{tabular}{|c|} 
Alcohol \\
Drug
\end{tabular} & Research_1A \\
\hline 315.1 & Arithmetical Disorder & $\begin{array}{l}\text { Neurotic disorders, personality } \\
\text { disorders, and oth }\end{array}$ & $\mathrm{MH}$ & $\begin{array}{l}\text { Specific Delays in } \\
\text { Development }\end{array}$ & Mathematics Disorder & & & Developmental Delays \\
\hline 315.2 & Oth Learning Difficulty & $\begin{array}{l}\text { Neurotic disorders, personality } \\
\text { disorders, and oth }\end{array}$ & $\mathrm{MH}$ & $\begin{array}{l}\text { Specific Delays in } \\
\text { Development }\end{array}$ & $\begin{array}{l}\text { Disorder of Written } \\
\text { Expression }\end{array}$ & & & Developmental Delays \\
\hline 315.3 & UNKNOWN & $\begin{array}{l}\text { Neurotic disorders, personality } \\
\text { disorders, and oth }\end{array}$ & $\mathrm{MH}$ & $\begin{array}{l}\text { Specific Delays in } \\
\text { Development }\end{array}$ & & & & Developmental Delays \\
\hline 315.31 & Development Language Dis & $\begin{array}{l}\text { Neurotic disorders, personality } \\
\text { disorders, and oth }\end{array}$ & $\mathrm{MH}$ & $\begin{array}{l}\text { Specific Delays in } \\
\text { Development }\end{array}$ & $\begin{array}{l}\text { Expressive Language } \\
\text { Disorder }\end{array}$ & & & Developmental Delays \\
\hline 315.32 & Receptive Language Disrd & $\begin{array}{l}\text { Neurotic disorders, personality } \\
\text { disorders, and oth }\end{array}$ & $\mathrm{MH}$ & $\begin{array}{l}\text { Specific Delays in } \\
\text { Development }\end{array}$ & $\begin{array}{l}\text { Mixed Receptive- } \\
\text { Expressive Language } \\
\text { Disorder }\end{array}$ & & & Developmental Delays \\
\hline 315.39 & Speech Language Dis Nec & $\begin{array}{l}\text { Neurotic disorders, personality } \\
\text { disorders, and oth }\end{array}$ & $\mathrm{MH}$ & $\begin{array}{l}\text { Specific Delays in } \\
\text { Development }\end{array}$ & Phonological Disorder & & & Developmental Delays \\
\hline 315.4 & Coordination Disorder & $\begin{array}{l}\text { Neurotic disorders, personality } \\
\text { disorders, and oth }\end{array}$ & $\mathrm{MH}$ & $\begin{array}{l}\text { Specific Delays in } \\
\text { Development }\end{array}$ & $\begin{array}{l}\text { Developmental } \\
\text { Coordination Disorder }\end{array}$ & & & Developmental Delays \\
\hline 315.5 & Mixed Development Dis & $\begin{array}{l}\text { Neurotic disorders, personality } \\
\text { disorders, and oth }\end{array}$ & $\mathrm{MH}$ & $\begin{array}{l}\text { Specific Delays in } \\
\text { Development }\end{array}$ & & & & Developmental Delays \\
\hline 315.8 & Development Delays Nec & $\begin{array}{l}\text { Neurotic disorders, personality } \\
\text { disorders, and oth }\end{array}$ & $\mathrm{MH}$ & $\begin{array}{l}\text { Specific Delays in } \\
\text { Development }\end{array}$ & & & & Developmental Delays \\
\hline 315.9 & Development Delay Nos & $\begin{array}{l}\text { Neurotic disorders, personality } \\
\text { disorders, and oth }\end{array}$ & $\mathrm{MH}$ & $\begin{array}{l}\text { Specific Delays in } \\
\text { Development }\end{array}$ & Learning Disorder NOS & & & Developmental Delays \\
\hline 315.90 & $\begin{array}{l}\text { Invalid Code }-315.9=\text { Development } \\
\text { Delay Nos }\end{array}$ & $\begin{array}{l}\text { Neurotic disorders, personality } \\
\text { disorders, and oth }\end{array}$ & $\mathrm{MH}$ & $\begin{array}{l}\text { Specific Delays in } \\
\text { Development }\end{array}$ & & & & Developmental Delays \\
\hline$\frac{316}{317}$ & Psychic Factor W Oth Dis & $\begin{array}{l}\text { Neurotic disorders, personality } \\
\text { disorders, and oth }\end{array}$ & $\mathrm{MH}$ & Personality Disorders & $\begin{array}{l}\text {...[Specified } \\
\text { Psychological Factor] } \\
\text { Affecting...[Indicate the } \\
\text { General Medical } \\
\text { Condition] }\end{array}$ & & & $\begin{array}{l}\text { Psychological Factors } \\
\text { Affecting Medical } \\
\text { Conditions }\end{array}$ \\
\hline 317 & Mild Mental Retardation & Mental retardation & $\mathrm{MH}$ & Mental Retardation & Mild Mental Retardation & & & Mental Retardation \\
\hline 317.0 & $\begin{array}{l}\text { Invalid Code - } 317=\text { Mild Mental } \\
\text { Retardation }\end{array}$ & Mental retardation & $\mathrm{MH}$ & Mental Retardation & & & & Mental Retardation \\
\hline 318 & Other Specified Mental Retardation & Mental retardation & $\mathrm{MH}$ & Mental Retardation & & & & Mental Retardation \\
\hline 318.0 & Mod Mental Retardation & Mental retardation & $\mathrm{MH}$ & Mental Retardation & $\begin{array}{l}\text { Moderate Mental } \\
\text { Retardation }\end{array}$ & & & Mental Retardation \\
\hline 318.1 & Severe Mental Retardat & Mental retardation & $\mathrm{MH}$ & Mental Retardation & $\begin{array}{l}\text { Severe Mental } \\
\text { Retardation }\end{array}$ & & & Mental Retardation \\
\hline 318.10 & $\begin{array}{l}\text { Invalid Code }-318.1=\text { Severe } \\
\text { Mental Retardat }\end{array}$ & Mental retardation & $\mathrm{MH}$ & Mental Retardation & & & & Mental Retardation \\
\hline 318.2 & Profound Mental Retardat & Mental retardation & $\mathrm{MH}$ & Mental Retardation & $\begin{array}{l}\text { Profound Mental } \\
\text { Retardation }\end{array}$ & & & Mental Retardation \\
\hline 319 & Mental Retardation Nos & Mental retardation & $\mathrm{MH}$ & Mental Retardation & $\begin{array}{l}\text { Mental Retardation, } \\
\text { Severity Unspecified }\end{array}$ & & & Mental Retardation \\
\hline
\end{tabular}

